Canad. J. Math. Vol. 51 (4), 1999 pp. 673-744

\title{
Brownian Motion and Harmonic Analysis on Sierpinski Carpets
}

\author{
Martin T. Barlow and Richard F. Bass
}

\begin{abstract}
We consider a class of fractal subsets of $\mathbb{R}^{d}$ formed in a manner analogous to the construction of the Sierpinski carpet. We prove a uniform Harnack inequality for positive harmonic functions; study the heat equation, and obtain upper and lower bounds on the heat kernel which are, up to constants, the best possible; construct a locally isotropic diffusion $X$ and determine its basic properties; and extend some classical Sobolev and Poincaré inequalities to this setting.
\end{abstract}

\section{Introduction}

We begin by considering a class of fractal subsets of $\mathbb{R}^{d}$ formed by the following generalization of the construction of the Cantor ternary set. Let $d \geq 2$ and let $F_{0}=[0,1]^{d}$. Let $l_{F} \geq 3$ be an integer and divide $F_{0}$ into $\left(l_{F}\right)^{d}$ equal subcubes. Next remove a symmetric pattern of subcubes from $F_{0}$ and call what remains $F_{1}$. Now repeat the procedure: divide each subcube that is contained in $F_{1}$ into $l_{F}^{d}$ equal parts, remove the same symmetric pattern from each as was done to obtain $F_{1}$ from $F_{0}$, and call what remains $F_{2}$. Continuing in this way we obtain a decreasing sequence of (closed) subsets of $[0,1]^{d}$. Let $F=\bigcap_{n=0}^{\infty} F_{n}$; we call $F$ a generalized Sierpinski carpet (GSC) or simply, a carpet. The standard SC (see [Sie]) is the GSC for which $d=2, l_{F}=3$, and $F_{1}$ consists of $F_{0}$ minus the central square. Let $m_{F}$ be the number of subcubes remaining in $F_{1}$, and let $d_{f}=\log m_{F} / \log l_{F}$. Then the Hausdorff dimension of $F$ is $d_{f}$. For an example of a GSC in $\mathbb{R}^{3}$, see the picture of the Menger sponge in [Man, p. 145].

We will also be interested in two other related sets, which have a large-scale structure similar to the small-scale structure of $F$. The first, which following [O1] we call the precarpet, is the set $\tilde{F}_{0}=\bigcup_{n=0}^{\infty} l_{F}^{n} F_{n}$. (Here and throughout this paper we write $\lambda G=\{\lambda x: x \in$ $G\}$ ). Note that $\tilde{F}_{0} \subset \mathbb{R}_{+}^{d}$, and that $\tilde{F}_{0} \cap\left[0, l_{F}^{n}\right]^{d}$ consists of $\left[0, l_{F}^{n}\right]^{d}$ with a number of (possibly adjacent) cubical holes removed, of sides varying from 1 to $l_{F}^{n-1}$. Write $\Gamma=\operatorname{int}\left(\tilde{F}_{0}\right)$ : then $\Gamma$ is a (non-empty) domain in $\mathbb{R}^{d}$ with a piecewise linear boundary-see Figure 2 . It is easy to check that $\Gamma$ satisfies the volume doubling condition. The second related set is the unbounded GSC $\tilde{F}=\bigcup_{n=0}^{\infty} l_{F}^{n} F$.

We may regard these sets as idealized models of a region with obstacles of many different sizes. Our purpose in this paper is to study the Laplace and heat equations on the spaces $F$, $\tilde{F}_{0}$, and $\tilde{F}$. In particular we:

\footnotetext{
Received by the editors July 16, 1998; revised August 19, 1998.

The first author's research was partially supported by a NSERC (Canada) grant. The second author's research was partially supported by NSF Grant DMS 9322689.

AMS subject classification: Primary: 60J60; secondary: 60B05, 60J35.

Keywords: Sierpinski carpet, fractal, Hausdorff dimension, spectral dimension, Brownian motion, heat equation, harmonic functions, potentials, reflecting Brownian motion, coupling, Harnack inequality, transition densities, fundamental solutions.

(c) Canadian Mathematical Society 1999.
} 

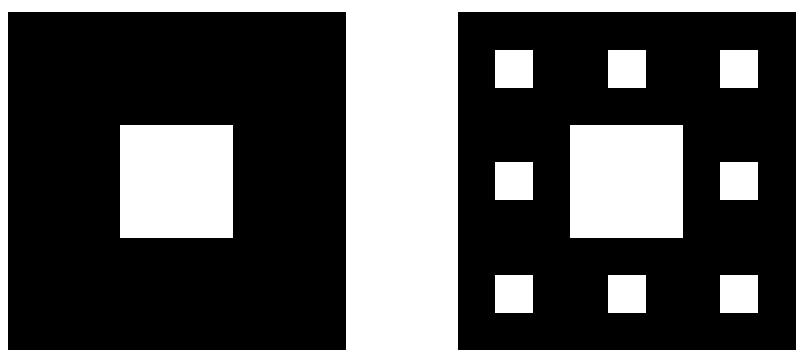

Figure 1: The first two stages of the construction of the standard Sierpinski carpet in two dimensions.

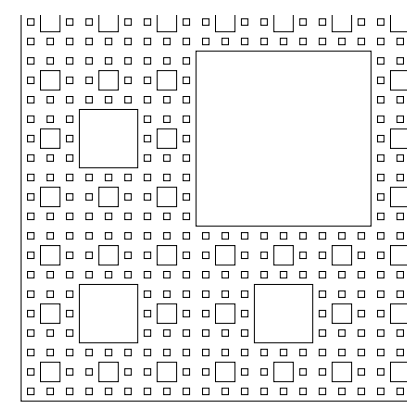

Figure 2: (Part of) the pre-carpet. The small squares have side 1.

(1) prove a uniform Harnack inequality for positive harmonic functions on $\tilde{F}_{0}$;

(2) study the heat equation on $\tilde{F}$ and $F$, and obtain upper and lower bounds on the heat kernel which are, up to constants, the best possible;

(3) construct a locally isotropic diffusion $X$ on $F$ and determine its basic properties;

(4) extend some classical Sobolev and Poincaré inequalities to this setting.

Just as the Euclidean dimension enters the standard heat kernel estimates and Sobolev inequalities, the Hausdorff dimension $d_{f}$ of $F$ plays a role in the analogues for $F$. What makes the fractal case intriguing, however, is that there is another parameter, called the spectral dimension $d_{s}$, which is much more significant. For example, the Sobolev inequality in $\mathbb{R}^{d}$ for $d>2$ states that if $p=2 d /(d-2)$ and $f$ and its gradient are in $L^{2}\left(\mathbb{R}^{d}\right)$, then

$$
\|f\|_{p} \leq c_{1}\|\nabla f\|_{2}
$$

The corresponding inequality for a GSC (see Theorem 1.5) has the $L^{p}$ norm of $f$ on the left hand side, but now with $p=2 d_{s} /\left(d_{s}-2\right)$; the dimension $d_{f}$ does not enter into the inequality.

The Hausdorff dimension of $F, d_{f}$ can be calculated easily from $l_{F}$ and the Lebesgue measure of $F_{1}$. On the other hand the spectral dimension $d_{s}$ appears to be a "physical" or "analytic" constant rather than a geometric one; we know of no simple formula for $d_{s}$ in terms of the geometry of $F$, and believe that none exists. Rather, $d_{s}$ is defined via the properties of harmonic functions on the sets $F_{n}$. While the exact determination of $d_{s}$ seems 
to be a hard problem, it is quite easy to obtain certain bounds, and we have in particular that $1<d_{s} \leq d_{f}<d$.

Any argument based purely on the geometry of $F$ will inevitably lead to results involving geometric constants. Since all the key inequalities relating to $F$ involve $d_{s}$, we cannot expect to be able to derive them by, say, starting with an isoperimetric inequality, as is possible in $\mathbb{R}^{d}$. Other methods suited to $\mathbb{R}^{d}$ also fail, and in fact, even very basic tools (like the cut-off functions used in Moser [M]) do not work.

We therefore had to develop some new techniques. Our basic approach is probabilistic: we construct a diffusion $X$ on $\tilde{F}$, which, because it is locally isotropic, we call a "Brownian motion" on $\tilde{F}$. We can then use properties of $X$ to derive bounds on its transition density (which solves the heat equation on $F$ ). Given these bounds, we can then derive Sobolev and Poincaré inequalities on $\tilde{F}$ and $\tilde{F}_{0}$.

The starting point of our analysis, and the hardest result in this paper, is a uniform (elliptic) Harnack inequality. Let $B$ be an open set in $\mathbb{R}^{d}$. We say that $h$ is harmonic on $B \cap F_{N}$ if $\Delta h(x)=0$ for $x \in B \cap \operatorname{int}\left(F_{N}\right)$, and the normal derivative of $h$ is 0 on $B \cap \partial F_{N}$ almost everywhere with respect to surface measure on $\partial F_{N}$. Write $B(x, r)$ for the usual open ball in $\mathbb{R}^{d}$ with centre $x$ and radius $r$.

Theorem 1.1 There exists $c_{1}$ not depending on $N$, such that if $x \in F_{N}, r>0$, and $h$ is positive and harmonic on $B(x, 2 r) \cap F_{N}$, then writing $A=B(x, r) \cap F_{N}$,

$$
\sup _{A} h(x) \leq c_{1} \inf _{A} h(y) .
$$

A similar result holds for the pre-carpet $\tilde{F}_{0}$.

Of course, since $F_{N}$ is a Lipschitz domain, for each $N$ the standard Harnack inequality guarantees there exists $c_{1}(N)$ such that (1.2) holds. The point of this theorem is that $c_{1}$ can be taken to be independent of $N$.

In an earlier paper [BB1] we proved a uniform Harnack inequality in the case $d=2$. The proof used a "path-crossing" argument which cannot be generalized to the case $d \geq 3$. Kusuoka and Zhou [KZ] extended this result to fractals satisfying $d_{s}<2$, but their method is also tied to the low-dimensional case. Standard approaches to Harnack inequalities in higher dimensions, such as Moser's iterative technique [M] or the NashFabes-Stroock method [FS], do not appear to work for GSCs, and we were therefore forced to use a different approach. Our proof of (1.2) uses the probabilistic technique of coupling. (See $[\mathrm{Lv}]$ for a general introduction to this method). More precisely, given distinct points $x, y \in F_{N}$, we construct two reflecting Brownian motions on $F_{N}$, starting at $x, y$, such that the two processes meet with a positive probability $p$ which is independent of $N$. Using this, we then establish a Harnack inequality with constants independent of $N$. We believe that our use of coupling to prove Harnack inequalities is new; it may also be applicable to a variety of other situations.

Given the Harnack inequality, we modify methods developed by us in earlier work on two-dimensional Sierpinski carpets (see [BB1]-[BB4]). We can construct the process $X$ as the limit of (suitably accelerated) reflecting Brownian motions on the sets $F_{N}$. Let $\mu$ be (a multiple of) the Hausdorff $x^{d_{f}}$-measure on $\tilde{F}$.

Theorem 1.2 There exists a nondegenerate continuous strong Markov process $X_{t}$ whose state space is $\tilde{F} . X_{t}$ has transition densities which have the strong Feller property and which are 
$\mu$-symmetric. The law of the process $\left(X_{t}, t \geq 0\right)$ is locally invariant under local isometries of $\tilde{F}$.

Write $P_{t}$ for the semigroup associated with $X$, and let $(\mathcal{L}, \mathcal{D}(\mathcal{L}))$ be the infinitesimal generator of $P_{t}$; we call $\mathcal{L}$ the Laplacian on $\tilde{F}$. The heat equation on $\tilde{F}$ then becomes

$$
\frac{\partial u}{\partial t}(x, t)=\mathcal{L} u(x, t), \quad x \in \tilde{F}, \quad t>0 .
$$

The fundamental solutions to the heat equation are given by the transition densities $p(t, x, y)$ for the process $X_{t}$ on $\tilde{F}$. The spectral dimension $d_{s}$ is defined from the sequence $R_{n}$ of electrical resistances across the sets $l_{F}^{n} F_{n}$. Let $d_{w}=2 d_{f} / d_{s}$; note that as $d_{s} \leq d_{f}$ we have $d_{w} \geq 2$.

Theorem $1.3 p(t, x, y)$ is symmetric and jointly continuous on $(0, \infty) \times \tilde{F} \times \tilde{F}$, and for each $x, y$ the function $p(t, x, y)$ is $C^{\infty}$ in $t$. There exist $c_{1}, c_{2}, c_{3}, c_{4}$ such that for all $x, y \in \tilde{F}$ and $t>0$,

$$
\begin{aligned}
& c_{1} t^{-d_{s} / 2} \exp \left(-c_{2}\left(\frac{|x-y|^{d_{w}}}{t}\right)^{1 /\left(d_{w}-1\right)}\right) \\
& \leq p(t, x, y) \leq c_{3} t^{-d_{s} / 2} \exp \left(-c_{4}\left(\frac{|x-y|^{d_{w}}}{t}\right)^{1 /\left(d_{w}-1\right)}\right) .
\end{aligned}
$$

Let $W_{t}$ be Brownian motion on the pre-carpet $\tilde{F}_{0}$, with normal reflection on $\partial \tilde{F}_{0}$, and let $q(t, x, y)$ be its transition density with respect to Lebesgue measure on $\tilde{F}_{0}$. These transition densities are the fundamental solutions to the heat equation $\partial u / \partial t=\frac{1}{2} \Delta u$ on $\tilde{F}_{0}$ with Neumann boundary conditions.

Since $\tilde{F}_{0}$ is locally similar to $\mathbb{R}^{d}$, but has a "fractal" global structure, we would expect $q(t, x, y)$ to have different behavior for small and large $t$. We would also expect, in view of standard large-deviation theory for Brownian motion, that, if $|x-y|$ is large in comparison with $t$ then $q(t, x, y)$ will exhibit Gaussian behavior. (Very roughly, if $|x-y|$ is much larger than $t$, then for the process $W$ to move from $x$ to $y$ in time $t$, it will with high probability stay close to the shortest path connecting $x$ and $y$, and it will have no time to feel the fractal structure of $\tilde{F}_{0}$ ).

Theorem 1.4 There exist $c_{1}, \ldots, c_{8} \in(0, \infty)$ such that if $x, y \in \tilde{F}_{0}$ and

(a) $t \geq \max (1,|x-y|)$, then

$$
\begin{aligned}
& c_{1} t^{-d_{s} / 2} \exp \left(-c_{2}\left(\frac{|x-y|^{d_{w}}}{t}\right)^{1 /\left(d_{w}-1\right)}\right) \\
& \leq q(t, x, y) \leq c_{3} t^{-d_{s} / 2} \exp \left(-c_{4}\left(\frac{|x-y|^{d_{w}}}{t}\right)^{1 /\left(d_{w}-1\right)}\right) ;
\end{aligned}
$$

(b) if $t \leq 1$, then

$$
c_{5} t^{-d / 2} \exp \left(-c_{6}|x-y|^{2} / t\right) \leq q(t, x, y) \leq c_{7} t^{-d / 2} \exp \left(-c_{8}|x-y|^{2} / t\right) .
$$


(c) if $t \geq 1,|x-y|>t$, then

$$
c_{5} t^{-d_{s} / 2} \exp \left(-c_{6}|x-y|^{2} / t\right) \leq q(t, x, y) \leq c_{7} t^{-d_{s} / 2} \exp \left(-c_{8}|x-y|^{2} / t\right) .
$$

As remarked above, the set $\tilde{F}_{0}$ satisfies the usual volume doubling condition and an elliptic Harnack inequality. However, since $q\left(t, x_{0},.\right)$ is parabolic on the whole space $(0, \infty) \times \tilde{F}_{0}$ it follows easily from the bounds in Theorem 1.4 that the (usual) parabolic Harnack inequality on $\tilde{F}_{0}$ fails for any GSC for which $d_{w}>2$. (See Proposition 7.11 for details, and Remark 5.4 for examples of GSCs for which it is known that $d_{w}>2$ ). This answers a question raised in Grigor'yan [Gr]. Essentially the point is that whereas an elliptic Harnack inequality such as Theorem 1.1 contains no information on the space-time scaling of the process $W$, this scaling information appears explicitly in the parabolic Harnack inequality.

To obtain the parabolic estimates above from Theorem 1.1 some additional information on the process $W$ is needed. This is provided by exploiting the close connection between resistances and crossing times. For graphs this was proved in [CRRST]—see also [Tet], and for Sierpinski carpets see [BB2], [BB4]. Using this, the resistance bounds in [BB4] and $[\mathrm{McG}]$, and the Harnack inequality, we can obtain good bounds on the time taken by $W$ to escape from a region of the form $B(x, r) \cap \tilde{F}_{0}$. These bounds then enable us to derive estimates for expressions like $\int_{0}^{\infty} e^{-\lambda t} t^{p} q(t, x, y) d t$ for suitable $p$, and using these we prove the upper bounds in Theorem 1.4. This method is also new and could easily be modified to give new proofs of the upper bounds of Aronson [A] for the heat kernels of uniformly elliptic operators in divergence form on $\mathbb{R}^{d}$.

For the key estimate for the lower bound for $q(t, x, y)$ we again use coupling; this may also have applications outside the fractal context. The bounds in Theorem 1.3 follow easily from those in Theorem 1.4

The relationship between bounds on the behavior of the norm of $P_{t}$ and Sobolev and other analytic inequalities has been explored very extensively in recent years, following the basic papers [V1] and [CKS]. Write $\|f\|_{p}^{p}=\int_{\tilde{F}}|f|^{p} d \mu$. As $X$ is $\mu$-symmetric, we can associate a Dirichlet form $(\mathcal{E}, \mathcal{D}(\mathcal{E}))$ with $P_{t}$ (see [FOT]). Since Theorem 1.3 implies easily that

$$
\left\|P_{t} f\right\|_{\infty} \leq c_{1} t^{-d_{s} / 2}\|f\|_{1},
$$

we can apply the theory mentioned above to immediately deduce a Sobolev inequality on $\tilde{F}$.

Theorem 1.5 Suppose $d_{s}>2$. There exists $c_{1}$ such that for all $f \in \mathcal{D}(\mathcal{E})$

$$
\|f\|_{p} \leq c_{1} \mathcal{E}(f, f)^{\frac{1}{2}}, \quad p=2 d_{s} /\left(d_{s}-2\right) .
$$

We also obtain a Sobolev inequality on $\tilde{F}_{0}$, and Poincaré and mass-capacity inequalities for both $\tilde{F}$ and $\tilde{F}_{0}$; see Section 7.

Let $G$ be the graph whose vertices are the centers of those unit squares that lie in $\tilde{F}_{0}$. Two vertices $x$ and $y$ will be connected by an edge if $|x-y|=1$. G is called the graphical SC. In [BB6] the results of this paper are used to obtain Poincaré, Sobolev, and mass-capacity inequalities for $G$ and to obtain transition probability estimates for the symmetric random walk on $G$.

Initial interest in the study of random walks or diffusions on fractals came from mathematical physicists working in the theory of disordered media—see [RT], [AO], and for a 
survey from a physical viewpoint [HBA]. The initial mathematical work was on the simplest non-trivial regular connected fractal, the Sierpinski gasket, in [Kus1], [Go], [BP]. In particular, $[\mathrm{BP}]$ obtained bounds similar to those in Theorem 1.3 for the transition densities of the Brownian motion on the Sierpinski gasket. The Sierpinski gasket $G$ is finitely ramified, that is, it can be disconnected by removing a finite number of points. This means that many subsets of $G$ have a finite boundary, and so a Harnack inequality can be proved in an elementary way. Subsequently many other finitely ramified fractals have been treated in a similar fashion—see for example [L], [Kig1], [Kig2], [Kum1], and [Fuk]. Note that while some of these papers use probability theory, others employ a purely analytic approach, and construct the Dirichlet form directly. However, no purely analytic derivation of the bounds on the heat kernel is known in the fractal context. While it possible for a finitely ramified fractal to have $d_{s}>2$ (see [Kum2] or [Ny]) these spaces are in some sense quite inhomogeneous. Indeed, Proposition 4.42 of [Bar1] implies that if bounds of the form (1.4) hold on a finitely ramified fractal then $d_{s}<2$. So if one wishes to study regular higher dimensional fractal spaces which are not simple products one is led to consider infinitely ramified fractals.

Generalized Sierpinski carpets provide a reasonably simple but general family of infinitely ramified fractals. See [GAM], [BAH] for early work by mathematical physicists, and [HHW1], [HHW2] for an approximate approach to the calculation of the spectral dimension. GSCs in two dimensions have been studied in [BB1], [BB2], [BBS], [BB3], [BB4], but as $d_{s} \leq d_{f}<d$ these sets also have $d_{s}<2$. There are only a few previous papers on GSCs with $d \geq 3$. The fractals studied by Kusuoka and Zhou in [KZ] include GSCs, and that paper contains a Harnack inequality similar to Theorem 1.1 above for GSCs with $d_{s}<2$. (They also have some results for more general GSCs). (See Section 9 of this paper for an example of a GSC with $d=3$ but $d_{s}<2$.) In addition, Osada [O1] has proved an isoperimetric inequality, and used it to deduce that

$$
q(t, x, y) \leq c_{1} t^{-d_{i} / 2}, \quad x, y \in \tilde{F}_{0}, \quad t \geq 1,
$$

where $d_{i}$ is the "isoperimetric dimension" of $\tilde{F}_{0}$. Comparing (1.8) with (1.5) we see that $d_{s} \geq d_{i}$, and we expect that in general the inequality is strict.

For surveys of work on finitely ramified fractals see [Kus2] or [Bar1], and for Sierpinski carpets see [Bas3].

The layout of this paper is as follows. Section 2 introduces the notation we will use together with a few basic facts. Section 3 contains the coupling argument: as this is quite long and hard we give here a very brief summary of the essential ideas. See also [BB5], where these results were announced.

Let $N \geq 0$. Let $S_{n}^{*}$ be the set of cubes of side length $2 l_{F}^{-n}$ with vertices in $2 l_{F}^{-n} Z^{d}$. Let us say $x \stackrel{m}{\sim} y$ if $x \in S_{1} \in \mathcal{S}_{m}^{*}, y \in S_{2} \in \mathcal{S}_{m}^{*}$, and there is an isometry from $S_{1}$ to $S_{2}$ that takes $x$ to $y$. Given $x \stackrel{m}{\sim} y$, we construct two reflecting Brownian motions $W_{t}^{x}$ and $W_{t}^{y}$ on $F_{N}$ starting from $x$ and $y$, respectively, such that $W_{t}^{x} \stackrel{m}{\sim} W_{t}^{y}$ for all $t$, and such that with some positive probability $c_{1}>0$ we have $W_{\sigma_{1}}^{x} \stackrel{m-1}{\sim} W_{\sigma_{1}}^{y}$, where $\sigma_{1}$ is the first time either $W^{x}$ or $W^{y}$ moves more than a few cubes in $\mathcal{S}_{m}$ away from their starting points. This construction uses the symmetry of $F_{1}$ very heavily. If $\left(n_{m}\right)$ is a sequence of integers and $\sigma_{n}$ is the $n$-th time $W^{x}$ or $W^{y}$ has moved more than a few cubes in $\mathcal{S}_{m}$, then a renewal-type argument tells us that 
$W^{x}\left(\sigma_{n_{m}}\right) \stackrel{m-1}{\sim} W^{y}\left(\sigma_{n_{m}}\right)$ with probability at least $1-\left(1-c_{1}\right)^{n_{m}}$. Repeating this argument for $m-1, m-2, \ldots$, we see that there is positive probability that $W^{x} \stackrel{0}{\sim} W^{y}$ at some time before either $W^{x}$ or $W^{y}$ hits $\partial F_{0}$.

In Section 4 we derive the uniform Harnack inequality from the coupling result. Section 5 contains the construction of the Brownian motion. In Section 6 we establish the bounds on the heat kernel for both $\tilde{F}$ and $\tilde{F}_{0}$, and in Section 7 we consider Sobolev and Poincaré inequalities. In Section 8 we establish a number of basic properties of the process $X_{t}$. These include transience and recurrence, moduli of continuity, the Hausdorff dimension of the range, the existence of local times, self-intersections, rates of escape, and a zero-one law. The paper is concluded in Section 9 by some examples and open problems.

\section{Notation and Preliminaries}

We begin by setting up our notation. We use the letter $c$ with subscripts to denote constants which depend only on the dimension $d$ and the carpet $F$. We renumber the constants for each lemma, proposition, theorem, and corollary.

Let $d \geq 2, F_{0}=[0,1]^{d}$, and let $l_{F} \in \mathbb{N}, l_{F} \geq 3$ be fixed. For $n \in \mathbb{Z}$ let $S_{n}$ be the collection of closed cubes of side $l_{F}^{-n}$ with vertices in $l_{F}^{-n} Z^{d}$. For $A \subseteq \mathbb{R}^{d}$, set

$$
\mathcal{S}_{n}(A)=\left\{S: S \subset A, S \in \mathcal{S}_{n}\right\} .
$$

For $S \in \mathcal{S}_{n}$, let $\Psi_{S}$ be the orientation preserving affine map which maps $F_{0}$ onto $S$.

We now define a decreasing sequence $\left(F_{n}\right)$ of closed subsets of $F_{0}$. Let $1 \leq m_{F}<l_{F}^{d}$ be an integer, and let $F_{1}$ be the union of $m_{F}$ distinct elements of $\mathcal{S}_{1}\left(F_{0}\right)$. We impose the following conditions on $F_{1}$ :

\section{Hypotheses 2.1}

(H1) (Symmetry) $F_{1}$ is preserved by all the isometries of the unit cube $F_{0}$.

(H2) (Connectedness) $\operatorname{Int}\left(F_{1}\right)$ is connected, and contains a path connecting the hyperplanes $\left\{x_{1}=0\right\}$ and $\left\{x_{1}=1\right\}$.

(H3) (Non-diagonality) Let $B$ be a cube in $F_{0}$ which is the union of $2^{d}$ distinct elements of $\mathcal{S}_{1}$. (So $B$ has side length $2 l_{F}^{-1}$ ). Then if $\operatorname{int}\left(F_{1} \cap B\right)$ is non-empty, it is connected.

(H4) (Borders included) $F_{1}$ contains the line segment $\left\{x: 0 \leq x_{1} \leq 1, x_{2}=\cdots=x_{d}=0\right\}$.

We may think of $F_{1}$ as being derived from $F_{0}$ by removing the interiors of $l_{F}^{d}-m_{F}$ squares in $\mathcal{S}_{1}\left(F_{0}\right)$. Given $F_{1}, F_{2}$ is obtained by removing the same pattern from each of the squares in $\mathcal{S}_{1}\left(F_{1}\right)$. Iterating, we obtain a sequence $\left(F_{n}\right)$, where $F_{n}$ is the union of $m_{F}^{n}$ squares in $\mathcal{S}_{n}\left(F_{0}\right)$. Formally, we define

$$
F_{n+1}=\bigcup_{S \in \mathcal{S}_{n}\left(F_{n}\right)} \Psi_{S}\left(F_{1}\right)=\bigcup_{S \in \mathcal{S}_{1}\left(F_{1}\right)} \Psi_{S}\left(F_{n}\right), \quad n \geq 1 .
$$

We call the set $F=\bigcap_{n=0}^{\infty} F_{n}$ a generalized Sierpinski carpet (GSC). Let $\operatorname{dim}(\cdot)$ denote Hausdorff dimension; by $[\mathrm{Hu}] \operatorname{dim}(F)=\log m_{F} / \log l_{F}$.

Remark 2.2 These conditions are natural higher-dimensional analogues of the ones given in $[B B 4,(2.1)]$. Since we are interested in constructing continuous processes on $F$, the hypothesis $(\mathrm{H} 2)$ is essential. It would be interesting to be able to consider GSCs for which 
the symmetry condition (H1) was either weakened or dispensed with entirely. However, (H1) plays a vital role in this paper, namely, in the key coupling argument in Section 3. Indeed, we do not expect the Harnack inequality Theorem 4.3 to remain true without strong symmetry assumptions on $F_{1}$.

The other two hypotheses, (H3) and (H4), are not so essential. We expect that results similar to those in this paper still hold for GSCs which do not satisfy (H3). However, the natural state space of the limiting process $X$ may no longer be $F$, and the added generality would significantly increase the complexity of the arguments. We include (H4) for simplicity-it ensures that the shortest path metric and the Euclidean metric on $F$ are comparable. See [BB4, Sect. 8] for some remarks on GSCs which do not satisfy (H4), and [FHK], [Kum1] for constructions of such a shortest path metric in the case of nested fractals.

We will be interested in unbounded analogues of $F$. Set $F_{k}=F_{0}$ for $k<0$ and for $n \in \mathbb{Z}$ let

$$
\tilde{F}_{n}=\bigcup_{r=0}^{\infty} l_{F}^{r} F_{n+r}
$$

and $\tilde{F}=\bigcap_{n=0}^{\infty} \tilde{F}_{n}$. In particular we call $\tilde{F}_{0}$ the pre-carpet (see [O1]). Let

$$
\mu_{n}(d x)=m_{F}^{n} 1_{\tilde{F}_{n}}(x) d x,
$$

and let $\mu$ be the weak limit of the $\mu_{n}$ : $\mu$ is a constant multiple of the Hausdorff $x^{\log m_{F}} / \log l_{F}$ measure on $\tilde{F}$.

We need notation for a block of cubes that have a point $x$ near the center. For $x=$ $\left(x_{1}, \ldots, x_{d}\right)$, let $\phi\left(x_{i}\right)$ be the integer $j$ such that $l_{F}^{-r}\left(j-\left(\frac{1}{2}\right)\right) \leq x_{i}<l_{F}^{-r}\left(j+\left(\frac{1}{2}\right)\right)$ and let

$$
D_{r}(x)=\left[\frac{\phi\left(x_{1}\right)-1}{l_{F}^{r}}, \frac{\phi\left(x_{1}\right)+1}{l_{F}^{r}}\right] \times \cdots \times\left[\frac{\phi\left(x_{d}\right)-1}{l_{F}^{r}}, \frac{\phi\left(x_{d}\right)+1}{l_{F}^{r}}\right] .
$$

Observe that $D_{r}(x)$ decreases as $r$ increases. Note also that $D_{r}(x)$ is a cube of side length $2 l_{F}^{-r}$

For distance on the sets $\tilde{F}_{n}$ we will frequently find it convenient to use the $\|\cdot\|_{\infty}$ norm, since in this norm the unit ball is a cube with sides parallel to the axes. We denote by $B_{\infty}(y, \varepsilon)$ the set $\left\{x \in \mathbb{R}^{d}:\|x-y\|_{\infty}<\varepsilon\right\}$, and use $B(x, \varepsilon)$ to denote the usual open balls in $\mathbb{R}^{d}$. Note the following:

\section{Lemma 2.3}

(a) If $y \in D_{n}(x)$ then $\|x-y\|_{\infty} \leq(3 / 2) l_{F}^{-n}$.

(b) If $\|x-y\|_{\infty} \leq(1 / 2) l_{F}^{-n}$ then $y \in D_{n}(x)$.

(c) If $x \in \tilde{F}$ and $n \in \mathbb{Z}$ then $m_{F}^{-n} \leq \mu\left(D_{n}(x)\right) \leq 2^{d} m_{F}^{-n}$

(d) If $x \in \tilde{F}_{0}$ and $n \leq 0$ then $m_{F}^{-n} \leq \mu_{0}\left(D_{n}(x)\right) \leq 2^{d} m_{F}^{-n}$.

(e) There exist constants $c_{1}, c_{2}$ such that for $x \in \tilde{F}_{0}$,

$$
\begin{gathered}
c_{1} r^{d} \leq \mu_{0}(B(x, r)) \leq c_{2} r^{d}, \quad 0<r<1, \\
c_{1} r^{d_{f}} \leq \mu_{0}(B(x, r)) \leq c_{2} r^{d_{f}}, \quad r>1 .
\end{gathered}
$$


(f) There exist constants $c_{1}, c_{2}$ such that for $x \in \tilde{F}$,

$$
c_{1} r^{d_{f}} \leq \mu(B(x, r)) \leq c_{2} r^{d_{f}}, \quad r>1 .
$$

In particular it follows immediately from (e) that $\left(\tilde{F}_{0},|\cdot-\cdot|, \mu_{0}\right)$ satisfies the volume doubling condition (see $[\mathrm{Gr}]$ ):

$$
\mu_{0}(B(x, r)) \leq c \mu_{0}(B(x, 2 r)), \quad \text { for } x \in \tilde{F}_{0}, r>0 .
$$

A similar volume doubling condition also holds for $\tilde{F}$.

We write

$$
H_{i}(t)=\left\{x \in \mathbb{R}^{d}: x_{i}=t\right\}, \quad t \in \mathbb{R} .
$$

We write $\mathcal{B}(G)$ for the Borel subsets of the set $G$. For a Borel set $A$ and process $X$ on $\mathbb{R}^{d}$ we write

$$
\mathcal{T}_{A}=\mathcal{T}(A)=\mathcal{T}_{A}(X)=\mathcal{T}_{A}^{X}=\inf \left\{t>0: X_{t} \in A\right\}
$$

for the hitting time of $A$ and

$$
\tau_{A}=\tau(A)=\tau_{A}(X)=\tau_{A}^{X}=\mathcal{T}\left(A^{c}\right)
$$

for the exit time of $A$. We also let

$$
\sigma_{r}(x)=\sigma_{r}^{X}(x)=\sigma_{r}(x, X)=\inf \left\{t>0: X_{t} \notin D_{r}(x)\right\} .
$$

We define the coupling time for two processes:

Definition 2.4 Let $X_{t}^{k}, k=1,2$, be processes on $\mathbb{R}^{d}$. The coupling time of $X^{1}, X^{2}$ is defined by

$$
T_{C}=T_{C}\left(X^{1}, X^{2}\right)=\inf \left\{t \geq 0: X_{t}^{1}=X_{t}^{2}\right\} .
$$

We say the $X^{k}$ are coupled if $X_{t}^{1}=X_{t}^{2}$ for $t \geq T_{C}$.

Let $D$ be an (open) Lipschitz domain in $\mathbb{R}^{d}$. We call a process $X_{t}$ a reflecting Brownian motion on $D$, or $\operatorname{RBM}(D)$ for short, if $X$ is a $\bar{D}$-valued diffusion which is locally a Brownian motion on $D$, with normal reflection on $\partial D$. If $D$ is a closed set in $\mathbb{R}^{d}, D=\overline{\operatorname{int}(D)}$, and $\operatorname{int}(D)$ satisfies the conditions above, then we say $X$ is a $\operatorname{RBM}(D)$ if $X$ is a $\operatorname{RBM}(\operatorname{int}(D))$. The existence (and uniqueness in law) of such processes is proved in [BH, Sect. 4], and [Bas1].

In the remainder of this section we will give some basic properties of $\operatorname{RBM}(D)$, where $D$ is a Lipschitz domain. We write $W$ for $\operatorname{RBM}\left(\tilde{F}_{0}\right)$.

In Section 3 we will make frequent use of the following fact.

Lemma 2.5 Let $D$ be a Lipschitz domain in $\mathbb{R}^{d}$, and let $X$ be a $\operatorname{RBM}(D)$. If $A \subset \mathbb{R}^{d}$ and $A$ is polar for standard d-dimensional Brownian motion, then $A \cap D$ is polar for $X$. 
Note in particular that subspaces of $\mathbb{R}^{d}$ of codimension greater than or equal to 2 are polar for $X$.

Proof Suppose $d \geq 3$ and $A$ is polar for $d$-dimensional Brownian motion. If $x \in \bar{D}$, then there exists $r_{x}>0$ such that $D \cap B\left(x, r_{x}\right)=D^{\prime} \cap B\left(x, r_{x}\right)$, where $D^{\prime}$ is the region above the graph of a Lipschitz function in some coordinate system. Since $\bar{D}$ can be covered by a countable union of balls of this kind and a countable union of polar sets is polar, it is sufficient to consider the case when $D$ is the region above the graph of a Lipschitz function and $A$ is bounded.

Let $v$ be the Green function for $X$ on $D$, and $w$ be the Green function for Brownian motion on $\mathbb{R}^{d}$. By $[\mathrm{BH}]$, Corollaries 3.3 and 3.5, there exist $c_{1}$ and $c_{2}$ such that

$$
c_{1}|y-z|^{2-d} \leq v(y, z) \leq c_{2}|y-z|^{2-d}, \quad y, z \in D
$$

Suppose $\nu$ is a non-zero measure supported on $A$ with $\int \nu(y, z) \nu(d z) \leq 1$ for all $y$. Then $\int w(y, z) \nu(d z)$ is bounded, which implies that $A$ is not polar for $d$-dimensional Brownian motion. So no such measure $\nu$ exists and $A$ is polar for $X$.

If $d=2$, we consider $\tilde{X}_{t}=\left(X_{t}, Z_{t}\right)$ in $D \times \mathbb{R}$, where $Z_{t}$ is an independent onedimensional Brownian motion and use the above argument to show that $A \times \mathbb{R}$ is polar for $\tilde{X}$ since it is polar for 3-dimensional Brownian motion.

We need to extend some results that were proved in $[\mathrm{BH}]$ for RBM in regions above the graph of a Lipschitz function to RBM in $\tilde{F}_{0}$. We begin with a support theorem for $\operatorname{RBM}\left(\tilde{F}_{0}\right)$.

Proposition 2.6 Let $\varepsilon>0$ and let $\psi:[0,1] \rightarrow \tilde{F}_{0}$ be a differentiable curve. There exists $c_{1}>0$ depending only on $\varepsilon$ and $\left\|\psi^{\prime}\right\|_{\infty}$ such that

$$
\mathbb{P}^{\psi} \psi(0)\left(\sup _{s \leq 1}\left|W_{s}-\psi(s)\right|<\varepsilon\right) \geq c_{1}
$$

Proof Let $x=\psi(0)$. By Proposition 3.6 of [BH] and scaling, there exist $\delta_{1}$ and $\delta_{2}$ depending on $\varepsilon$ and $\left\|\psi^{\prime}\right\|_{\infty}$ such that with probability at least $c_{2}$ we have $\sup _{s \leq \delta_{1}}\left|W_{s}-\psi(s)\right|<\varepsilon / 3$ and $\operatorname{dist}\left(W_{\delta_{1}}, \partial D\right)>\delta_{2}$. By the Markov property, it thus suffices to show

$$
\mathbb{P}^{y}\left(\sup _{\delta_{1} \leq s \leq 1}\left|W_{s}-\psi(s)\right|<\varepsilon\right) \geq c_{3}
$$

when $\left|y-\psi\left(\delta_{1}\right)\right|<\varepsilon / 3$ and $\operatorname{dist}(y, \partial D)>\delta_{2} / 2$. However (2.9) follows by applying the support theorem for standard $d$-dimensional Brownian motion [Bas2, p. 59] with $\varepsilon$ replaced by $\varepsilon / 3$ and $\psi$ replaced by a curve $\bar{\psi}$ starting at $y$ that is always within $\varepsilon / 3$ of $\psi$ but such that $\bar{\psi}$ never gets closer than a distance $\left(\varepsilon \wedge \delta_{2}\right) / 2$ to $\partial D$.

Fix $x_{0} \in \tilde{F}_{0}$. Let $m \in \mathbb{Z}$. As $\tilde{F}_{0} \cap D_{m}\left(x_{0}\right)$ is a bounded Lipschitz domain, by Lemma 4.3 of $[\mathrm{BH}]$ and its proof, a $\operatorname{RBM}\left(\tilde{F}_{0} \cap D_{m}\left(x_{0}\right)\right) W$ has a continuous transition density $\bar{q}_{D_{m}\left(x_{0}\right)}(t, x, y)=\bar{q}(t, x, y)$ with respect to Lebesgue measure on $\tilde{F}_{0} \cap D_{m}\left(x_{0}\right)$. By Theorem 2.3 and Remark 3.11 of $[\mathrm{BH}]$,

$$
\bar{q}(t, x, y) \leq c_{1}(m) t^{-d / 2} \exp \left(-c_{2}(x-y)^{2} / t\right), \quad x, y \in \tilde{F}_{0} \cap D_{m}\left(x_{0}\right), \quad t \leq 1 .
$$


Since $\bar{q}$ is the transition density of a symmetric process, $\bar{q}(t, x, y)=\bar{q}(t, y, x)$. By [Bas2, Sect. 4], there is an eigenvalue expansion for $\bar{q}$ :

$$
\bar{q}(t, x, y)=\sum_{i=1}^{\infty} e^{-\lambda_{i} t} \bar{\varphi}_{i, m}(x) \bar{\varphi}_{i, m}(y),
$$

where the convergence is absolute and uniform. This and (2.10) imply

$$
\bar{q}(t, x, y) \leq c_{3} t^{-d / 2} \exp \left(-c_{4} t\right), \quad x, y \in \tilde{F}_{0} \cap D_{m}\left(x_{0}\right), \quad t>0,
$$

where $c_{3}$ and $c_{4}$ depend on $m$. Cauchy-Schwarz and (2.11) imply

$$
\bar{q}(t, x, y) \leq \bar{q}(t, x, x)^{\frac{1}{2}} \bar{q}(t, y, y)^{\frac{1}{2}}
$$

and also that $\bar{q}(t, x, x)$ is nonincreasing in $t$ for each $x \in \tilde{F}_{0}$.

\section{Coupling of Brownian Motions}

The coupling argument given in this section is the foundation of the results in this paper. The argument is quite long, and requires several steps. A great deal of notation, especially for various special subsets of $\mathbb{R}^{d}$, will be required; however, none of the notation introduced in this section will be used elsewhere. The reader may find it helpful to focus on the case $d=3$.

Lemma 3.1 (A Reflection Principle) Let $D^{0}$ be a Lipschitz domain in $\mathbb{R}^{d}$, let $D=\overline{D^{0}}$ and let $W$ be a $\operatorname{RBM}(D)$. Let $H$ be a hyperplane, $g: \mathbb{R}^{d} \rightarrow \mathbb{R}^{d}$ be reflection across $H$, and $J_{1}, J_{2}$ be the two half spaces determined by $H$. Let $A \subset \partial D$, and $B_{1}, B_{2}$ be subsets of $A$.

Suppose that

(i) $g: D \rightarrow D$

(ii) $g\left(B_{1}\right)=B_{2}$

(iii) $B_{i} \subseteq \overline{J_{i}}, \quad i=1,2$

(iv) $g\left(A \cap J_{1}\right) \subset A$.

Then

$$
\mathbb{P}^{x}\left(W_{\mathcal{T}_{A}} \in B_{1}\right) \geq \mathbb{P}^{x}\left(W_{\mathcal{T}_{A}} \in B_{2}\right) \quad \text { for } x \in J_{1} \cap D \text {. }
$$

Proof Let $C_{2}=A \cap J_{2}-B_{2}$, and $C_{1}=g\left(C_{2}\right)$. Note that $C_{1}$ is not necessarily contained in $A$. Write $u_{i}(x)=\mathbb{P}^{x}\left(W_{\mathcal{T}_{A}} \in B_{i}\right)$, and $v=u_{2}-u_{1}$. Since $W_{t}$ killed on hitting $A$ has continuous paths, the maximum principle holds for $v$. Let $\beta=\sup _{x \in J_{1} \cap D} v(x)$. Suppose $\beta>0$. As $v(x) \leq 0$ for $x \in A \cap J_{1}$, by the maximum principle we have

$$
\sup _{x \in J_{1} \cap D} v(x)=\sup _{y \in H \cap D} v(y) .
$$


Set $S=\mathcal{T}_{B_{1}} \wedge \mathcal{T}_{B_{2}} \wedge \mathcal{T}_{C_{1}} \wedge \mathcal{T}_{C_{2}}$. Then for $y \in H \cap D$,

$$
u_{i}(y)=\mathbb{P}^{y}\left(W_{S} \in B_{i}\right)+\mathbb{E}^{y} 1_{\left(W_{S} \in C_{1}\right)} u_{i}\left(W_{S}\right), \quad i=1,2 .
$$

Since by symmetry $\mathbb{P}^{y}\left(W_{S} \in B_{1}\right)=\mathbb{P}^{y}\left(W_{S} \in B_{2}\right)$, it follows that

$$
v(y)=\mathbb{E}^{y} 1_{\left(W_{S} \in C_{1}\right)} v\left(W_{S}\right) .
$$

However, again by symmetry, $\mathbb{P}^{y}\left(W_{S} \in C_{1}\right)=\mathbb{P}^{y}\left(W_{S} \in C_{2}\right)$, and so $\mathbb{P}^{y}\left(W_{S} \in C_{1}\right) \leq 1 / 2$. Hence for $y \in H \cap D, v(y) \leq \mathbb{P}^{y}\left(W_{S} \in C_{1}\right) \beta \leq \beta / 2$. Thus $\beta \leq \beta / 2$, a contradiction to our assumption that $\beta>0$.

The following definition of a sequence of stopping times will be used several times.

Definition 3.2 Let $\mathcal{H}=\left\{H_{\alpha}, \alpha \in I\right\}$ be a family of closed non-empty subsets of $\mathbb{R}^{d}$ with the property that $\left\{\alpha: H_{\alpha} \cap B(x, 1) \neq \varnothing\right\}$ is finite for all $x$. (Note this implies that $I$ is countable). Write $H=\bigcup_{\alpha \in I} H_{\alpha}$. Let $\left(X_{t}, t \geq 0\right)$ be a continuous process on $\mathbb{R}^{d}$ such that $H_{\alpha} \cap H_{\beta}$ is polar for $X$, for all $\alpha, \beta \in I$. Then successive disjoint hits by $X$ on $\mathcal{H}$ are the sequence of stopping times $\left(T_{n}, n \geq 0\right)$ defined by:

$$
\begin{gathered}
T_{0}=\inf \left\{t \geq 0: X_{t} \in H\right\}, \\
\Gamma_{n}=\left\{\alpha \in I: X_{T_{n}} \in H_{\alpha}\right\}, \\
T_{n+1}=\inf \left\{t \geq T_{n}: X_{t} \in H-\bigcup_{\alpha \in \Gamma_{n}} H_{\alpha}\right\} .
\end{gathered}
$$

Lemma 3.3 Let $\mathcal{H}, X,\left(T_{n}\right)$ be as above. Then $\lim _{n \rightarrow \infty} T_{n}=+\infty$, a.s.

Proof Suppose $T_{n}<\infty$. Since $B\left(X_{T_{n}}(\omega), 1\right)$ intersects at most finitely many $H_{\alpha}$, $\Gamma_{n}$ is a finite set. (In fact, if $T_{n}>0$ then as $H_{\alpha} \cap H_{\beta}$ is polar, $\Gamma_{n}$ contains only one element). Therefore $X_{T_{n}}(\omega)$ is a positive distance from $H-\bigcup_{\alpha \in \Gamma_{n}} H_{\alpha}$. Hence $T_{n+1}>T_{n}$. Now suppose $\lim _{n} T_{n}(\omega)=S(\omega)<\infty$. Then as $\left\{\alpha: H_{\alpha} \cap B\left(X_{S}(\omega), 1\right) \neq \varnothing\right\}$ is finite, there must exist $\alpha, \beta \in I$ such that $X_{T_{n}}(\omega) \in H_{\gamma}$ for infinitely many $n$, for $\gamma=\alpha$, $\beta$. So $X_{S}(\omega) \in H_{\alpha} \cap H_{\beta}$, and hence, by the polarity assumption, we deduce $\mathbb{P}(S<\infty)=0$.

Definition 3.4 Let $D=F_{n_{0}}$, for some $n_{0} \geq 1$, and let $W$ be $\operatorname{RBM}(D)$. We define

$$
\begin{gathered}
H_{i j}=\left\{x: x_{i}+x_{j}=1\right\} \\
L_{i}=H_{i}(0) \cap[0,1 / 2]^{d}, \\
M_{i j}=\left\{x \in[0,1]^{d}: x_{i}=0,1 / 2 \leq x_{j} \leq 1,0 \leq x_{k} \leq 1 / 2, k \neq j\right\}, \quad \text { for } i \neq j, \\
\tau=\inf \left\{t \geq 0: W_{t} \in \bigcup_{i=1}^{d} H_{i}(1)\right\} .
\end{gathered}
$$


The hyperplane $H_{i}(t)$ was defined in (2.3). For any hyperplane $H \subset \mathbb{R}^{d}$, let $g_{H}: \mathbb{R}^{d} \rightarrow \mathbb{R}^{d}$ be reflection in $H$. If $x=\left(x_{1}, \ldots, x_{d}\right)$ note that

$$
\begin{gathered}
g_{H_{i}\left(\frac{1}{2}\right)}(x)=\left(x_{1}, \ldots, x_{i-1}, 1-x_{i}, x_{i+1}, \ldots, x_{d}\right), \\
g_{H_{12}}(x)=\left(1-x_{2}, 1-x_{1}, x_{3}, \ldots, x_{d}\right) .
\end{gathered}
$$

We now use the symmetry of $D$, and the invariance of $W$ under certain isometries of $D$, to deduce lower bounds for certain hitting probabilities of $W$. (These are higherdimensional analogues of the "corner" and "knight's" moves in [BB1]). In what follows we use $q_{i}$ to denote strictly positive reals which depend only on the dimension $d$.

Proposition 3.5 Let $i, j \in\{1, \ldots, d\}$. Then

$$
\mathbb{P}^{x}\left(\mathcal{T}_{L_{j}} \leq \tau\right) \geq q_{1}>0, \quad \text { for } x \in L_{i} .
$$

Proof Set $T=\mathcal{T}\left(H_{j}(0) \cup \bigcup_{k=1}^{d} H_{k}(1)\right)$. We will actually prove that $\mathbb{P} x\left(W_{T} \in L_{j}\right) \geq q_{1}$ for $x \in L_{i}$. By the symmetry of $D$ we can take $j=1, i=2$. (If $i=j$ the result is trivial). Fix $x \in L_{2}$.

Now apply Lemma 3.1 with $H=H_{1}(1 / 2), A=\left(H_{1}(0) \cup \bigcup_{k=1}^{d} H_{k}(1)\right) \cap D, B_{1}=$ $H_{1}(0) \cap D, B_{2}=H_{1}(1) \cap D, J_{1}=\left\{x: x_{1}<1 / 2\right\}, J_{2}=\left\{x: x_{1}>1 / 2\right\}$. Hypotheses (3.1)(i)(iv) are easily verified, $L_{2} \subset J_{1} \cap D$, and so we deduce

$$
\mathbb{P}^{x}\left(W_{T} \in H_{1}(0)\right) \geq \mathbb{P}^{x}\left(W_{T} \in H_{1}(1)\right) .
$$

We now use Lemma 3.1 again, with $H=H_{1 k}(k \neq 1), A$ as before, $B_{1}=H_{1}(0) \cap D$, $B_{2}=H_{k}(1) \cap D, J_{1}=\left\{x: x_{1}+x_{k}<1\right\}, J_{2}=\mathbb{R}^{d}-\left(H \cup J_{1}\right)$. Once again (3.1)(i)-(iv) are easily verified, and so

$$
\mathbb{P}^{x}\left(W_{T} \in H_{1}(0)\right) \geq \mathbb{P}^{x}\left(W_{T} \in H_{k}(1)\right), \quad k \neq 1 .
$$

Combining (3.5) and (3.6), and using that fact that

$$
\mathbb{P}^{x}\left(W_{T} \in H_{1}(0)\right)+\sum_{k=1}^{d} \mathbb{P}^{x}\left(W_{T} \in H_{k}(1)\right) \geq 1,
$$

we obtain

$$
\mathbb{P}^{x}\left(W_{T} \in H_{1}(0)\right) \geq(1+d)^{-1}
$$

Now set $G_{n}=\left(H_{1}(0) \cap D\right) \cap \bigcap_{k=2}^{n}\left\{x_{k} \leq 1 / 2\right\}$, for $2 \leq n \leq d$. Write $G_{1}=H_{1}(0)$. Let $1 \leq n \leq d-1$, set $G_{n}^{\prime}=\overline{G_{n-1}-G_{n}}$, and apply Proposition 3.1 with $H=H_{n}(1 / 2)$, $B_{1}=G_{n}, B_{2}=G_{n}^{\prime}, J_{1}=\left\{x: x_{n}<1 / 2\right\}, J_{2}=\left\{x: x_{n}>1 / 2\right\}$, and $A$ as before. We deduce that

$$
\mathbb{P}^{x}\left(W_{T} \in G_{n}\right) \geq \mathbb{P}^{x}\left(W_{T} \in G_{n}^{\prime}\right) .
$$




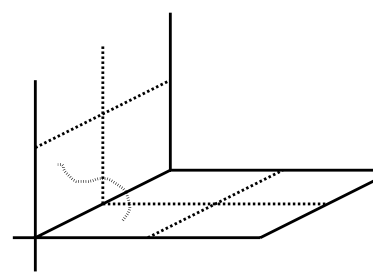

Figure 3: A corner move.

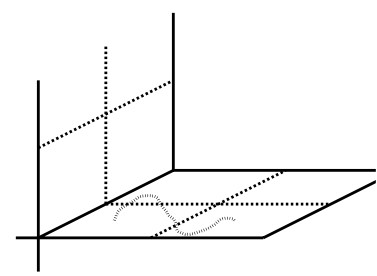

Figure 4: A slide.

Hence

$$
2 \mathbb{P}^{x}\left(W_{T} \in G_{n}\right) \geq \mathbb{P}^{x}\left(W_{T} \in G_{n}\right)+\mathbb{P}^{x}\left(W_{T} \in G_{n}^{\prime}\right) \geq \mathbb{P}^{x}\left(W_{T} \in G_{n-1}\right) .
$$

Since $G_{d}=L_{1}$,

$$
\mathbb{P}^{x}\left(W_{T} \in L_{1}\right) \geq 2^{-(d-1)} \mathbb{P}^{x}\left(W_{T} \in H_{1}(0)\right),
$$

and this proves the proposition with $q_{1}^{-1}=(1+d) 2^{d-1}$.

Remark 3.6 We call a piece of the path of $W$ in which it moves from $L_{i}$ to $L_{j}$ a corner move. The other kind of move we will need is from $L_{i}$ to $M_{i j}$, which we will call a slide. The next few results lead up to the proof of the following.

Proposition 3.7 Let $1 \leq i_{0}, j_{0} \leq d$, with $i_{0} \neq j_{0}$. Then

$$
\mathbb{P}^{x}\left(\mathcal{T}_{M_{i_{0} j_{0}}} \leq \tau\right) \geq q_{2}>0, \quad \text { for } x \in L_{i_{0}}
$$

By symmetry it is sufficient to prove this in the case $i_{0}=1, j_{0}=2$. Write $\Lambda_{i}^{0}=$ $\left\{0 \leq x_{i} \leq 1 / 2\right\}, \Lambda_{i}^{1}=\left\{1 / 2 \leq x_{i} \leq 1\right\}$, and let

$$
\begin{gathered}
K_{1}^{l}=D \cap H_{1}(1) \cap \Lambda_{2}^{l}, \quad l=0,1, \\
K_{j}^{l}=D \cap H_{j}(1) \cap \Lambda_{1}^{l}, \quad l=0,1, \quad 2 \leq j \leq d, \\
\Gamma=\bigcup_{i=1}^{d} H_{i}(1), \\
K=H_{1}(0) \cap \Lambda_{2}^{1} \cap D .
\end{gathered}
$$

Lemma 3.8 $\mathbb{P}^{\mathrm{p} x}\left(\mathcal{T}_{M_{12}} \leq \tau\right) \geq 2^{2-d} \mathbb{P}^{x}\left(\mathcal{T}_{K} \leq \tau\right), x \in L_{1}$. 
Proof Set $G_{d+1}=K$, and let

$$
G_{n}=K \cap \bigcap_{i=n}^{d} \Lambda_{i}^{0}, \quad 1 \leq n \leq d
$$

So $G_{3}=D \cap H_{1}(0) \cap \Lambda_{2}^{1} \cap \bigcap_{3}^{d} \Lambda_{i}^{0}=M_{12}$. Now let $3 \leq n \leq d$, and apply Proposition 3.1 with $H=H_{n}(1 / 2), B_{1}=G_{n}, B_{2}=g_{H}\left(G_{n}\right), J_{1}=\left\{x_{n} \leq 1 / 2\right\}, J_{2}=\left\{x_{n}>1 / 2\right\}$, and $A=\Gamma \cup B_{1} \cup B_{2}=\Gamma \cup G_{n+1}$.

We verify (3.1)(i)-(iv). (i) and (ii) are obvious, while (iii) is immediate from the observation $G_{n} \subset \Lambda_{n}^{0} \subset \overline{J_{1}}$. To prove (3.1)(iv) let $x \in A \cap J_{1}$. If $x \in B_{1}, g_{H}(x) \in B_{2}$, so suppose $x \in \Gamma$. Then $x \in \bigcup_{i \neq n} H_{i}(1)$, and so $g_{H}(x) \in \bigcup_{i \neq n} H_{i}(1)$. Then $g_{H}\left(A \cap J_{1}\right) \subset A$, as required. Finally, note that $L_{1} \subset J_{1}$. So by (3.2),

$$
\mathbb{P}^{x}\left(W_{\mathcal{T}_{A}} \in G_{n}\right) \geq \mathbb{P}^{x}\left(W_{\mathcal{T}_{A}} \in g_{H}\left(G_{n}\right)\right), \quad x \in L_{1}
$$

Hence, since $G_{n+1}=G_{n} \cup g_{H}\left(G_{n}\right)$, we deduce

$$
\mathbb{P}^{x}\left(W_{\mathcal{T}\left(\Gamma \cup G_{n+1}\right)} \in G_{n}\right) \geq \frac{1}{2} \mathbb{P}^{x}\left(W_{\mathcal{T}\left(\Gamma \cup G_{n+1}\right)} \in G_{n+1}\right)=\frac{1}{2} \mathbb{P}^{x}\left(\mathcal{T}_{G_{n+1}}<\mathcal{T}_{\Gamma}\right)
$$

Since $\mathbb{P}^{x}\left(\mathcal{T}_{G_{n}}<\mathcal{T}_{\Gamma}\right) \geq \mathbb{P}^{\mathrm{p} x}\left(W_{\mathcal{T}_{\Gamma \cup G_{n+1}}} \in G_{n}\right)$, it follows that $\mathbb{P}^{\mathrm{p} x}\left(\mathcal{T}_{G_{n}}<\mathcal{T}_{\Gamma}\right) \geq$ $(1 / 2) \mathbb{P}^{x}\left(\mathcal{T}_{G_{n+1}}<\mathcal{T}_{\Gamma}\right)$ for $3 \leq n \leq d$, and the result follows immediately.

Lemma 3.9 Set $A=K \cup \bigcup_{i=1}^{d} H_{i}(1)$. For $x \in L_{1}$,

(a) $\mathbb{P}^{\mathrm{P} x}\left(W_{\mathcal{T}_{A}} \in K\right) \geq \mathbb{P}^{\mathrm{p} x}\left(W_{\mathcal{T}_{A}} \in K_{2}^{0}\right)$,

(b) $\mathbb{P}^{x}\left(W_{\mathcal{T}_{A}} \in K_{2}^{0}\right) \geq \mathbb{P}^{x}\left(W_{\mathcal{T}_{A}} \in K_{2}^{1}\right)$.

Proof These follow from Proposition 3.1 with $H=H_{12}$, for (a), and with $H=H_{1}(1 / 2)$, for (b).

Lemma 3.10 $\mathbb{P}^{x}\left(W_{\tau} \in H_{2}(1)\right) \geq q_{3}>0$ for $x \in L_{1}$.

Proof For $1 \leq i \leq d$ set

$$
F_{i}=\left\{W \text { hits } L_{2}, L_{3}, \ldots, L_{d} \text { in order before } \tau \text {, and } W_{\tau} \in H_{i}(1)\right\}
$$

Let $F=\bigcup_{1 \leq i \leq d} F_{i}$. By Proposition $3.5 \mathrm{P}^{x x}(F) \geq q_{1}^{d-1}$. Set $q_{4}=q_{1}^{d-1}$. Now let $N$ be a random variable independent of $\left(W_{t}, t \geq 0\right)$ with $\mathbb{P}(N=i)=(d-1)^{-1}$ for $i \in$ $\{1,3, \ldots, d\}$.

Let $\Lambda_{i}=\left\{x: x_{2}=x_{i}\right\}$ for $i \neq 2$, let $g_{i}=g_{\Lambda_{i}}$, and define

$$
W_{t}^{*}= \begin{cases}W_{t} & 0 \leq t \leq \mathcal{T}_{\Lambda_{N}} \\ g_{N}\left(W_{t}\right) & \mathcal{T}_{\Lambda_{N}} \leq t\end{cases}
$$


Then $W^{*}$ is also a $\operatorname{RBM}(D)$. Note that $\mathcal{T}_{\Lambda_{N}} \leq \max _{i \neq 2} \mathcal{T}_{\Lambda_{i}}<\tau$ on $F$, and that on $F_{k} \cap\{N=k\}, W_{\tau}^{*}=g_{N}\left(W_{\tau}\right)=g_{k}\left(W_{\tau}\right)$, so that as $g_{k}: H_{k}(1) \rightarrow H_{2}(1)$, we have $W_{\tau}^{*} \in$ $\mathrm{H}_{2}(1)$. Therefore

$$
\begin{aligned}
\mathbb{P}^{x}\left(W_{\tau} \in H_{2}(1)\right) & =\mathbb{P}^{x}\left(W_{\tau}^{*} \in H_{2}(1)\right) \\
& \geq \mathbb{P}^{x}\left(\bigcup_{k \neq 2} F_{k} \cap\{N=k\}\right) \\
& =\sum_{k \neq 2} \mathbb{P}^{x}\left(F_{k}\right) \cdot \mathbb{P}(N=k) \\
& =(d-1)^{-1} \mathbb{P}^{x}(F) \geq(d-1)^{-1} q_{4} .
\end{aligned}
$$

Proof of Proposition 3.7 From Lemma 3.10 we have, writing $A=K \cup \bigcup_{i=1}^{d} H_{i}(1)$,

$$
\begin{aligned}
q_{3} & \leq \mathbb{P}^{x}\left(W_{\tau} \in H_{2}(1)\right) \\
& =\mathbb{P}^{x}\left(W_{\tau} \in H_{2}(1), \mathcal{T}_{K}<\tau\right)+\mathbb{P}^{x}\left(W_{\tau} \in H_{2}(1), \mathcal{T}_{K}=\tau\right) \\
& =\mathbb{P}^{x}\left(W_{\mathcal{T}_{A}} \in K\right)+\mathbb{P}^{x}\left(W_{\mathcal{T}_{A}} \in H_{2}(1)\right) .
\end{aligned}
$$

From Lemma 3.9,

$$
\mathbb{P}^{x}\left(W_{\mathcal{T}_{A}} \in H_{2}(1)\right) \leq \mathbb{P}^{x}\left(W_{\mathcal{T}_{A}} \in K_{2}^{0}\right)+\mathbb{P}^{x}\left(W_{\mathcal{T}_{A}} \in K_{2}^{1}\right) \leq 2 \mathbb{P}^{x}\left(W_{\mathcal{T}_{A}} \in K\right) .
$$

So Proposition 3.7 follows, with $q_{2}=q_{3} / 3$.

Definition 3.11 A set $A \subset \mathbb{R}^{d}$ is a half-face if there exists $i \in\{1, \ldots, d\}, a=\left(a_{1}, \ldots, a_{d}\right) \in$ $\frac{1}{2} \mathbb{Z}^{d}$ with $a_{i} \in \mathbb{Z}$ such that

$$
A=\left\{x: x_{i}=a_{i}, a_{j} \leq x_{j} \leq a_{j}+1 / 2 \text { for } j \neq i\right\} .
$$

For $A$ as above set $\iota(A)=i$. Let $\mathcal{A}$ be the collection of half-faces, and set

$$
A^{*}=\bigcup\{A: A \in \mathcal{A}\}, \quad A_{0}^{*}=\bigcup\{A \cap B ; A, B \in \mathcal{A}, A \neq B\} .
$$

Note that $\operatorname{dim}\left(A_{0}^{*}\right)=d-2$, so that $A_{0}^{*}$ is polar for $\operatorname{RBM}(D)$ by Lemma 2.5 for any Lipschitz domain $D \subset \mathbb{R}^{d}$. Recall the definition of $\tilde{F}_{0}$. Set

$$
\mathcal{A}_{F}=\left\{A \in \mathcal{A}: A \subset \tilde{F}_{0}\right\} .
$$

We define a graph structure on $\mathcal{A}$ by taking $\{A, B\}$ to be an edge if

$$
\operatorname{dim}(A \cap B)=d-2, \quad \text { and } \quad A \cup B \subset C \quad \text { for some } C \in \mathcal{S}_{0} .
$$

Let $\mathcal{E}(\mathcal{A})$ be the set of edges, and let

$$
\mathcal{E}\left(\mathcal{A}_{F}\right)=\left\{\{A, B\} \in \mathcal{E}(\mathcal{A}): A, B \in \mathcal{A}_{F}\right\}
$$

be the edges in the subgraph $\left(\mathcal{A}_{F}, \mathcal{E}\left(\mathcal{A}_{F}\right)\right)$. Write $d_{F}$ for the natural graph distance on $\mathcal{A}_{F}$. Since $\tilde{F}_{0}$ is connected we deduce immediately

Lemma 3.12 The graph $\left(\mathcal{A}_{F}, \mathcal{E}\left(\mathcal{A}_{F}\right)\right)$ is connected. 
We will need terminology for the various types of edges in $\mathcal{E}(\mathcal{A})$. We call an edge $\{A, B\}$ an $i-j$ corner if $\iota(A)=i, \iota(B)=j$, and $i \neq j$ and call $\{A, B\}$ an $i-j$ slide if $\iota(A)=$ $\iota(B)=i$, and the line joining the centers of $A$ and $B$ is parallel to the $x_{j}$ axis. We say two edges are of the same type if they are both $i-j$ corners, or both $i-j$ slides, for some pair $(i, j)$. Note that the move $\left(L_{i}, L_{j}\right)$ is an $i-j$ corner, and $\left(L_{i}, M_{i j}\right)$ is an $i-j$ slide.

Now set

$$
\begin{gathered}
\mathcal{A}_{F}^{(2)}=\left\{(A, B) \in \mathcal{A}_{F}: \iota(A)=\iota(B)\right\}, \\
\mathcal{E}\left(A_{F}^{(2)}\right)=\left\{\left\{\left(A, A^{\prime}\right),\left(B, B^{\prime}\right)\right\}:\{A, B\},\left\{A^{\prime}, B^{\prime}\right\}\right. \text { are edges } \\
\text { of the same type in } \left.\mathcal{E}\left(\mathcal{A}_{F}\right)\right\} .
\end{gathered}
$$

The graph $\left(\mathcal{A}_{F}^{(2)}, \mathcal{E}\left(\mathcal{A}_{F}^{(2)}\right)\right)$ is not connected, and this will cause us some additional trouble. Write $d_{F}^{(2)}$ for the graph distance (with values in $\mathbb{Z}_{+} \cup\{+\infty\}$ ) on $\mathcal{A}_{F}^{(2)}$.

Let $g_{i}^{R}: \mathbb{R}^{d} \rightarrow \mathbb{R}^{d}$ be reflection in the hyperplane $H_{i}(0)$, and let $\mathcal{G}_{R}$ be the group generated by the $g_{i}^{R}$; thus $\mathcal{G}_{R}$ is the set of transformations that can be obtained by a sequence of reflections parallel to the axes. Note that $\mathcal{G}_{R}$ is commutative. For $n \in \mathbb{Z}$ let $\mathcal{S}_{n}^{*}$ be the collection of cubes of side $2 l_{F}^{-n}$ with vertices in $2 l_{F}^{-n} \mathbb{Z}^{d}$. For $C \in \mathcal{S}_{n}^{*}$ let $\psi_{C}$ be the translation which maps $\mathcal{C}$ onto $\left[-l_{F}^{-n}, l_{F}^{-n}\right]^{d}$, and let

$$
\mathcal{G}(C, D)=\left\{\psi_{D}^{-1} \circ g \circ \psi_{C}: g \in \mathcal{G}\right\} .
$$

For $x, y \in \mathbb{R}^{d}$ write $x \stackrel{n}{\sim} y$ if there exist $C, D \in \mathcal{S}_{n}^{*}$, and $g \in \mathcal{G}(C, D)$ such that $x \in C$, $y \in D$, and $g(x)=y$. Similarly, for $A, B \in \mathcal{A}$ write $A \stackrel{n}{\sim} B$ if there exist $C, D \in \mathcal{S}_{n}^{*}$, and $g \in \mathcal{G}(C, D)$, such that $g(A)=B$. We write $\sim$ for $\stackrel{0}{\sim}$.

Proposition 3.13 Let $A, B \in \mathcal{A}_{F}$, with $A \sim B$. Let $C_{0} \in \mathcal{S}\left(\tilde{F}_{0}\right)$ with $A \subset C_{0}$. Suppose $B=B_{0}, B_{1}, \ldots, B_{n}=A$ is a chain in $\mathcal{A}_{F}$ with $\left\{B_{i-1}, B_{i}\right\} \in \mathcal{E}\left(\mathcal{A}_{F}\right)$ for $1 \leq i \leq n$. Then there exist $A_{0}, A_{1}, \ldots, A_{n}$ in $\mathcal{A}_{F}$ such that

(a) $\left\{\left(A_{i-1}, B_{i-1}\right),\left(A_{i}, B_{i}\right)\right\} \in \mathcal{E}\left(\mathcal{A}_{F}^{(2)}\right)$ for $1 \leq i \leq n$.

(b) $A_{i} \subset C_{0}$ for $0 \leq i \leq n$.

(c) $A_{i} \sim B_{i}$ for $0 \leq i \leq n$.

(d) $\left(A_{0}, B_{0}\right)=(A, B),\left(A_{n}, B_{n}\right)=(A, A)$.

In particular, $d_{F}^{(2)}((A, B),(A, A))=d_{F}(A, B)$.

Proof Let $C_{1}$ be a cube in $\mathcal{S}_{0}\left(\tilde{F}_{0}\right)$ such that $B_{0} \cup B_{1} \subset C_{1}$. For $i=0,1$, let $D_{i}$ be the unique cube in $\mathcal{S}_{0}^{*}$ containing $C_{i}$. Let $A_{0}=A$. As $A_{0} \sim B_{0}$, there exists $g \in \mathcal{G}\left(D_{0}, D_{1}\right)$ with $g\left(A_{0}\right)=B_{0}$. If $g\left(C_{0}\right) \neq C_{1}$, then since $B_{0} \subset C_{1} \cap g\left(C_{0}\right)$ there exists an isometry $h \in \mathcal{G}\left(D_{1}, D_{1}\right)$ mapping $g\left(C_{0}\right)$ to $C_{1}$ and preserving $B_{0}$. If $C_{1}=g\left(C_{0}\right)$ take $h$ to be the identity. Let $g^{\prime}=h \circ g$ : then $g^{\prime}\left(A_{0}\right)=B_{0}$, and $g^{\prime}\left(C_{0}\right)=C_{1}$. Set $A_{1}=\left(g^{\prime}\right)^{-1}\left(B_{1}\right)$. Since $A_{1} \subset C_{0} \subset \tilde{F}_{0}, A_{1} \in \mathcal{A}_{F}$. It is clear that the edges $\left(A_{0}, A_{1}\right),\left(B_{0}, B_{1}\right)$ in $\mathcal{A}_{F}$ are of the same type; therefore $\left\{\left(A_{0}, A_{1}\right),\left(B_{0}, B_{1}\right)\right\} \in \mathcal{E}\left(\mathcal{A}_{F}^{(2)}\right)$. Also, since $g^{\prime}\left(A_{1}\right)=B_{1}$, we have $A_{1} \sim B_{1}$.

Continuing in this way we can construct a sequence $A_{i}, 0 \leq i \leq n$, in $\mathcal{A}_{F}$ satisfying conditions (a), (b), (c) above. To prove (d), note that since $A_{n} \sim B_{n}=A$, we have $A_{n} \sim A$. However, since $A$ and $A_{n}$ are both contained in $C_{0}$, this implies that $A_{n}=A$. 
This argument also proves that $d_{F}^{(2)}((A, B),(A, A)) \leq d_{F}(A, B)$; the reverse inequality is evident.

Let

$$
\widehat{F}_{N}=\bigcup_{x \in \mathbb{Z}^{d}}\left(x+F_{N}\right)
$$

Let $G \subset \mathbb{R}^{d}$ be a union (finite or infinite) of cubes in $\mathcal{S}_{0}$ : we will assume $G$ is connected. We now construct a reflecting Brownian motion on $G \cap \widehat{F}_{N}$ from a driving process $\xi$ on $\widehat{F}_{N}$. We begin with a deterministic construction.

Let $\xi(t), t \geq 0$ be a continuous path on $\widehat{F}_{N}$ with $\xi(0)=z$ which satisfies the conditions $\xi(t) \notin A_{0}^{*}$ for any $t \geq 0$. Let $x \in G \cap \widehat{F}_{N}$ with $x \sim z$, and let $\eta=\left(\eta_{0}, \eta_{1}, \ldots\right) \in\{0,1\}^{Z_{+}}$. We construct from $\xi$ and $\eta$ a continuous path $w(t)$ on $\tilde{F}_{N}$, with $w(0)=x$.

Let $T_{n}, n \geq 0$ be successive disjoint hits by $\xi(\cdot)$ on $\mathcal{A}$. (We can of course take the process $X$ in Definition 3.2 to be deterministic.) If $z \notin A^{*}$ then $T_{0}>0$. Then each of $z, x$ lies in exactly one cube in $\mathcal{S}_{0}^{*}$ : call these cubes $D^{x}, D^{z}$, and let $\psi \in \mathcal{G}\left(D^{z}, D^{x}\right)$ be such that $\psi(z)=x$. Define

$$
w(t)=\psi(\xi(t)), \quad 0 \leq t \leq T_{0} .
$$

Let $z^{\prime}=\xi\left(T_{0}\right), x^{\prime}=w\left(T_{0}\right)$; we have $x^{\prime}, z^{\prime} \in A^{*}-A_{0}^{*}$. Thus each of $x^{\prime}, z^{\prime}$ lies in exactly two cubes in $S_{0}$ : call these cubes $S_{0}^{x}, S_{1}^{x}, S_{0}^{z}, S_{1}^{z}$. Using lexicographic ordering of the cubes in $\mathcal{S}_{0}$, we can ensure these labels are uniquely specified. As $x^{\prime} \in G$, there are two possibilities:

(i) Exactly one of $S_{0}^{x}, S_{1}^{x}$ is contained in $G$,

(ii) $S_{0}^{x}, S_{1}^{x}$ are both contained in $G$.

For $y=x, z, i=0,1$ let $D_{i}^{y} \in \mathcal{S}_{0}^{*}$ satisfy $S_{i}^{y} \subset D_{i}^{y}$. For $i, j=0,1$ there exists a unique map $\psi_{i j} \in \mathcal{G}\left(D_{i}^{z}, D_{j}^{x}\right)$ such that $\psi_{i j}\left(z^{\prime}\right)=x^{\prime}$, and $\psi_{i j}\left(S_{i}^{z}\right)=S_{j}^{x}$. The uniqueness is evident; the existence is proved as in Proposition 3.13. Note that $\psi_{0 j}=\psi_{1 j}$ on $S_{0}^{z} \cap S_{1}^{z}$.

(i) Let $S_{i}^{x} \subset G$, so (int $\left.S_{1-i}^{x}\right) \cap G=\varnothing$. Now define

$$
\varphi: S_{0}^{z} \cup S_{1}^{z} \rightarrow S_{i}^{x} \quad \text { by } \varphi=\left.\psi_{i j}\right|_{S_{j}^{z}} \quad \text { for } j=0,1,
$$

and let $w(t)=\varphi(\xi(t)), T_{0} \leq t \leq T_{1}$.

(ii) For $k=0,1$ define $\varphi_{k}: S_{0}^{z} \cup S_{1}^{z} \rightarrow S_{0}^{x} \cup S_{1}^{x}$ by

$$
\varphi_{k}=\left.\psi_{i j}\right|_{S_{j}^{z}}, \quad i=0,1 ;
$$

here $j=j(i, k)=i+k(\bmod 2)$. Set $w(t)=\varphi_{\eta_{0}}(\xi(t)), T_{0} \leq t \leq T_{1}$.

Note that in either case, for each $t \in\left[T_{0}, T_{1}\right]$, there exists $i$, $j$ such that $w(t)=\psi_{i j}(\xi(t))$, so that $w(t) \sim \xi(t)$ for $T_{0} \leq t \leq T_{1}$. (We also have $w(t) \sim \xi(t)$ on the initial segment $\left.\left[0, T_{0}\right].\right)$

The same construction can now be repeated on each of the time intervals $\left[T_{i-1}, T_{i}\right]$, using, as above, the index $\eta_{i-1}$ to make a choice of maps each time case (ii) arises. The path $w \in C\left(\mathbb{R}_{+}, G \cap \widehat{F}_{N}\right)$ is a function of $G, \xi, \eta$ and $x$ only; we write

$$
w=\Gamma_{0}(x, G, \xi, \eta) .
$$


We can now define a pair of Brownian motions on $\tilde{F}$. Recall the definition of $A_{0}^{*}$ from (3.9). The following theorem follows in a straightforward fashion from the properties of $\Gamma_{0}$.

Theorem 3.14 Let $\left(\Omega, \mathcal{F}, \mathcal{F}_{t}, \mathbb{P}^{\mathrm{P}}\right)$ be a probability space carrying a reflecting Brownian motion $\xi_{t}$ on $\widehat{F}_{N}$, and independent sequences $\left(\eta_{i}^{1}, i \geq 0\right),\left(\eta_{i}^{0}, i \geq 0\right)$ of i.i.d. Bernoulli random variables. Let each of $G_{k}, k=1,2$, be a union of cubes in $\mathcal{S}_{0}$. Suppose $\xi_{0}=z \notin A_{0}^{*}$, and let $x_{k} \in G_{k} \cap \widehat{F}_{N}$, satisfy $x_{1} \sim x_{2} \sim z$. Let $T_{i}, i \geq 0$ be successive disjoint hits by $\xi$ on $\mathcal{A}$, and suppose that $\eta_{i}^{k} \in \mathcal{F}_{T_{i}}, k=1,2, i \geq 0$. Set

$$
X_{t}^{k}=\Gamma_{0}\left(x_{k}, G_{k}, \xi, \eta^{k}\right), \quad k=1,2 .
$$

Then

(a) $X^{k}$ is a $\operatorname{RBM}\left(G_{k} \cap \hat{F}_{N}\right)$, with $X_{0}^{k}=x_{k}$.

(b) $X_{t}^{k} \sim \xi_{t}$ for $t \geq 0$.

(c) $X^{1}$ and $X^{2}$ are conditionally independent given $\xi$.

(d) If $T_{i}\left(X^{k}\right), i \geq 0$ denote successive disjoint hits by $X^{k}$ on $\mathcal{A}$, then $T_{i}\left(X^{k}\right)=T_{i}$ for $i \geq 0$.

Proof Note that $A_{0}^{*}$ is polar for $\xi_{t}$, so that $\left(T_{i}\right)$ and $X^{k}$ are well-defined. (b), (c), (d) are all evident from the definition of $\Gamma_{0}$.

For (a), let $S_{j}^{x}, S_{j}^{z}$ denote the cubes in $\mathcal{S}_{0}$ given in the construction of $\Gamma_{0}$. Fix $k$. We have

$$
X_{t}^{k}=\varphi\left(\xi_{t}\right), \quad 0 \leq t \leq T_{1}
$$

where $\varphi: S_{0}^{z} \cup S_{1}^{z} \rightarrow \tilde{F}_{N}$. In case (3.14)(ii) $\varphi$ is an isometry between $\left(S_{0}^{z} \cup S_{1}^{z}\right) \cap \hat{F}_{N}$ and $\left(S_{0}^{x_{k}} \cup S_{1}^{x_{k}}\right) \cap \widehat{F}_{N}$, so that $X_{t}^{k}$ is a $\operatorname{RBM}\left(\widehat{F}_{N}\right)$ on the time interval $\left[0, T_{1}\right]$. In case $(3.14)(\mathrm{i})$, if $S_{k}^{x_{1}} \subset \tilde{F}_{0}$, then $\varphi$ can be written in the form $\varphi=\varphi_{0} \circ \varphi_{1}$, where $\varphi_{1}$ is reflection in the hyperplane containing $S_{0}^{x_{k}} \cap S_{1}^{x_{k}}$, and $\varphi_{0}$ is an isometry of $\mathbb{R}^{d}$. Again, it is clear that $X_{t}$ is a $\operatorname{RBM}\left(\widehat{F}_{N}\right)$ on $\left[0, T_{1}\right]$.

Remark We will call a pair of processes defined in this way linked RBMs.

The next sequence of results will extend the lower bounds on the probabilities of certain moves, given in Propositions 3.3 and 3.7, to joint moves by a pair of RBM $X^{k}$, defined by (3.16). We begin by introducing some further notation.

Definition 3.15 Let $J=\{-1,1\}^{d}$, and let $\mathcal{E}(J)=\{\{x, y\} \in J:|x-y|=2\}$. Then $(J, \mathcal{E}(J))$ is the natural graph of vertices of the hypercube. For $a=\left(a_{1}, \ldots, a_{d}\right) \in J$, let

$$
C_{a}=\left\{x \in[-1,1]^{d}: 0 \leq x_{i} a_{i} \leq 1\right\}
$$

be the portion of $[-1,1]^{d}$ that is in the orthant determined by $a$. For example, if $d=3$ and $a=(1,-1,1)$ then

$$
C_{a}=[0,1] \times[-1,0] \times[0,1] .
$$

Let $J_{1}, J_{2}$ be non-empty, connected subsets of $J$, and let

$$
D_{i}=\bigcup_{a \in J_{i}}\left(C_{a} \cap \hat{F}_{N}\right), \quad i=1,2 .
$$


For $e=\{a, b\} \in \mathcal{E}(J)$, let

$$
G_{e}=C_{a} \cap C_{b}, \quad G=\bigcup_{e \in \mathcal{E}(J)} G_{e}, \quad L_{e}=G_{e} \cap\left[-\frac{1}{2}, \frac{1}{2}\right]^{d} .
$$

Thus $L_{e} \in \mathcal{A}$; for $j \neq \iota\left(L_{e}\right)$ let $M_{e j}$ be the unique half face contained in $G_{e}$ which is obtained by translating $L_{e}$ a distance $1 / 2$ parallel to the $j$-axis.

We remark that

$$
\begin{gathered}
L_{e} \sim L_{f} \text { if and only if } \iota\left(L_{e}\right)=\iota\left(L_{f}\right), \\
M_{e i} \sim M_{f j} \text { if and only if } i=j \text { and } \iota\left(L_{e}\right)=\iota\left(L_{f}\right) .
\end{gathered}
$$

Now let $x_{k} \in D_{k}, k=1,2$, satisfy $x_{1} \sim x_{2}$, with $x_{k} \notin A_{0}^{*}$, let $\eta^{k}, \xi,\left(\mathcal{F}_{t}\right)$ be as in Theorem 3.14, and let

$$
X^{k}=\Gamma_{0}\left(x_{k}, D_{k}, \xi, \eta^{k}\right), \quad k=1,2,
$$

be a pair of linked RBMs on $D_{1}, D_{2}$, respectively. Let

$$
\tau=\inf \left\{t \geq 0: \xi_{t} \in \partial[-1,1]^{d}\right\}
$$

and let $\left(T_{n}, n \geq 0\right)$ be successive disjoint hits by $\xi$ on $\mathcal{H}=\left\{G_{e}, e \in \mathcal{E}(J)\right\} \cup\left\{\partial[-1,1]^{d}\right\}$. Note that $\tau=T_{M}$ for some (random) $M \geq 0$. Let $\pi:[-1,1]^{d} \rightarrow[0,1]^{d}$ be defined by $\pi\left(x_{1}, \ldots, x_{d}\right)=\left(\left|x_{1}\right|, \ldots,\left|x_{d}\right|\right)$. Since

$$
\pi\left(X_{t}^{1}\right)=\pi\left(X_{t}^{2}\right)=\pi\left(\xi_{t}\right), \quad 0 \leq t \leq \tau,
$$

we see that if $\left(T_{n}^{k}, n \geq 0, k=1,2\right)$ are successive disjoint hits by $X^{k}$ on $\mathcal{H}$, then $T_{n}^{k}=T_{n}$ for $0 \leq n \leq M$.

We now define a number of processes associated with $X^{k}$ and $\xi$. For $n<M$, let $I_{n} \in$ $\{1, \ldots, d\}$ be such that $\xi_{T_{n}} \in H_{I_{n}}(0)$, and for $a \in J$ let $A_{n}(a)$ be the unique element of $J$ such that $C_{a} \cap C_{A_{n}(a)} \subset H_{I_{n}}(0)$. Set $\mathcal{G}_{t}=\sigma\left(\xi_{s}, s \leq t\right)$; note that $I_{n}$ and $A_{n}(a)$ are $\mathcal{G}_{T_{n}}$ measurable on $\{n<M\}$. For each $0 \leq n<M, X_{T_{n}}^{k}$ lies in exactly two of the cubes $C_{a}$, while $X_{T_{M}}^{k}$ lies in exactly one cube, a.s. For $1 \leq n<M$ let $Z_{n}^{k}$ be the unique element of $J_{k}$ such that $C_{Z_{n}^{k}} \supset\left\{X_{T_{n-1}}^{k}, X_{T_{n}}^{k}\right\}$. Choose $a_{k}$ such that $x_{k} \in C_{a_{k}}$ and let $Z_{0}^{k}=a_{k}$.

Set, for $n \geq 0$,

$$
\begin{gathered}
p_{n}(a, b)=\mathbb{P}^{x}\left(Z_{n}^{1}=a, Z_{n}^{2}=b \mid \mathcal{G}_{T_{n}}\right) 1_{(n<M)}, \\
p_{n}^{k}(a)=\mathbb{P}^{x}\left(Z_{n}^{k}=a \mid \mathcal{G}_{T_{n}}\right) 1_{(n<M)} .
\end{gathered}
$$

Lemma 3.16 $p_{n}(a, b)=p_{n}^{1}(a) p_{n}^{2}(b)$.

Proof Write $\mathcal{F}_{t}^{(k)}$ for the natural filtration of $X^{k}$. Then $Z_{n}^{k} \in \mathcal{F}_{T_{n}}^{(k)}$, while $\{M<n\} \in$ $\mathcal{G}_{T_{n}}$. By (3.18) $\mathcal{F}_{t}^{(1)}$ and $\mathcal{F}_{t}^{(2)}$ are conditionally independent given $\mathcal{G}_{T_{n}}$; the result follows immediately. 
Now write

$$
V_{n}^{k}(a)=1_{J_{k}}\left(A_{n}^{k}(a)\right), \quad a \in J
$$

Lemma 3.17 For $a \in J_{k}$,

$$
p_{n+1}^{k}(a)=1_{(n+1<M)}\left(p_{n}^{k}(a)\left(1-V_{n}^{k}(a)\right)+\frac{1}{2}\left(p_{n}^{k}(a)+p_{n}^{k}\left(A_{n}(a)\right)\right) V_{n}^{k}(a)\right), \quad \text { a.s. }
$$

To simplify notation, in the next two proofs we will omit the superscript $k$ from $X$, $p_{n}$, etc.

Proof Note first that since $X_{T_{n}} \in C_{Z_{n}} \cap C_{A_{n}\left(Z_{n}\right)}$, we have $Z_{n+1} \in\left\{Z_{n}, A_{n}\left(Z_{n}\right)\right\}$. So also $Z_{n} \in\left\{Z_{n+1}, A_{n}\left(Z_{n+1}\right)\right\}$, and

$$
\left\{Z_{n}, A_{n}\left(Z_{n}\right)\right\}=\left\{Z_{n+1}, A_{n}\left(Z_{n+1}\right)\right\}, \quad \text { on }\{n+1<M\}
$$

Suppose first that $A_{n}(a) \notin J_{k}$. Then (3.22) implies that $Z_{n+1}=a$ and $n+1<M$ if and only if $Z_{n}=a$, and $n+1<M$. So

$$
1_{(n+1<M)}\left(1-V_{n}(a)\right) p_{n+1}(a)=1_{(n+1<M)}\left(1-V_{n}(a)\right), \quad \text { a.s. }
$$

Now suppose that $A_{n}(a) \in J_{k}$. Then to have $Z_{n+1}=a$ it is necessary that $X_{T_{n}} \in C_{a}$, while if $X_{T_{n}} \in C_{a}$ then $Z_{n+1}=a$ if and only if $n+1<M$ and $X_{T_{n+1}} \in C_{a}$. Since the set $C_{a} \cup C_{A_{n}(a)}$ is symmetric about the hyperplane $H_{I_{n}}(0)$, it follows that

$$
1_{(n+1<M)} V_{n}(a) p_{n+1}(a)=1_{(n+1<M)} V_{n}(a)\left(\frac{1}{2} p_{n}(a)+\frac{1}{2} p_{n}\left(A_{n}(a)\right)\right) .
$$

Combining (3.23) and (3.24) gives (3.21).

Now let $U_{n}^{k}=\left\{a: p_{n}^{k}(a)>0\right\}, u_{n}^{k}=\#\left(U_{n}^{k}\right)$, and

$$
q_{n}^{k}=1_{(n<M)} \min \left\{p_{n}(a): p_{n}(a)>0\right\} .
$$

Lemma 3.18 For $n \geq 0, q_{n}^{k} 1_{(n<M)} \geq 2^{-2^{d}} 1_{(n<M)}$.

Proof Note first that as $p_{0}\left(a_{k}\right)=1, q_{0}=1$. From (3.21) we have that on $\{n+1<M\}$ either $p_{n+1}(a)=p_{n}(a)$, or $p_{n+1}(a)=\frac{1}{2}\left(p_{n}(a)+p_{n}\left(A_{n}(a)\right)\right)$.

Suppose $n+1<M$, and $U_{n}=U_{n+1}$. Choose $a \in U_{n+1}$ such that $q_{n+1}=p_{n+1}(a)$. If $A_{n}(a) \notin J_{k}$, then $p_{n+1}(a)=p_{n}(a) \geq q_{n}$. If $A_{n}(a) \in J_{k}$ then $p_{n}\left(A_{n}(a)\right)>0$, since otherwise $p_{n+1}\left(A_{n}(a)\right)=p_{n}(a) / 2>0$, so that $A_{n}(a) \in U_{n+1}-U_{n}$. Therefore

$$
p_{n+1}(a) \geq \frac{1}{2}\left(p_{n}(a)+p_{n}\left(A_{n}(a)\right)\right) \geq q_{n}
$$


So if $U_{n}=U_{n+1}$ we have $q_{n+1} \geq q_{n}$, on $\{n+1<M\}$.

If $n+1<M$, and $U_{n} \neq U_{n+1}$, we have $u_{n+1}-u_{n} \geq 1$. Again choose $a$ such that $q_{n+1}=p_{n+1}(a)$; since at least one of $a$ or $A_{n}(a)$ must be in $U_{n}$ we deduce $q_{n+1} \geq q_{n} / 2$.

So, in all cases we have

$$
q_{n+1} \geq 2^{-\left(u_{n+1}-u_{n}\right)} q_{n} \quad \text { on }\{n+1<M\},
$$

and since $1 \leq u_{1} \leq u_{n} \leq 2^{d}$, for all $n$, the result follows.

For the $\operatorname{RBM}(\cdot) X^{k}$ defined above, and $A_{1}, A_{2} \in \mathcal{A}$, set

$$
T\left(A_{1}, A_{2}\right)=\inf \left\{t \geq 0:\left(X_{t}^{1}, X_{t}^{2}\right) \in\left(A_{1}, A_{2}\right)\right\} .
$$

Given two linked processes $X_{t}^{k}, k=1,2$, we let $\mathbb{P}^{\left(x_{1}, x_{2}\right)}$ denote the joint law of the pair $\left(X_{t}^{1}, X_{t}^{2}\right)$ with $X_{t}^{k}$ started at $x_{k}, k=1,2$. When the starting points are clear, we just write $\mathbb{P}$.

We can now give a lower bound on the probability of certain joint moves.

Theorem 3.19 There exists a constant $p_{1}=p_{1}(d)>0$, with the following properties. Suppose for $k=1,2, e_{k}, f_{k} \in \mathcal{E}(J), x_{k} \in L_{e_{k}}, x_{1} \sim x_{2}, x_{k} \notin A_{0}^{*}$, with $\iota\left(L_{f_{1}}\right)=\iota\left(L_{f_{2}}\right)=i$, and $G_{f_{k}} \subset D_{k}$. Then

(a) $\mathbb{P P}\left(T\left(L_{f_{1}}, L_{f_{2}}\right)<\tau\right) \geq p_{1}$.

(b) If $j \neq i$, then

$$
\mathbb{P}\left(T\left(M_{f_{1} j}, M_{f_{2} j}\right)<\tau\right) \geq p_{1} .
$$

Proof Let $\bar{\xi}_{t}=\pi\left(\xi_{t}\right)$; note that $T_{i}, 0 \leq i \leq M$, are also successive disjoint hits by $\bar{\xi}$ on $\mathcal{H}$. For $r \geq 1$ define sets $B_{r}$ by $B_{r}=H_{r}(0) \cap[0,1 / 2]^{d}, 1 \leq r \leq d$, and $B_{k d+r}=B_{r}$, for $k \geq 1,1 \leq r \leq d$. Let

$$
S_{0}=0, \quad S_{r+1}=\inf \left\{t \geq S_{r}: \bar{\xi}_{t} \in B_{r+1}\right\}, \quad r \geq 1 .
$$

Thus the stopping times $\left(S_{0}, S_{1}, \ldots\right)$ form a subsequence of $\left(T_{0}, T_{1}, \ldots\right)$. For $r \geq 0$, let $N_{r}$ be such that $T_{N_{r}}=S_{r}$.

Suppose that $S_{r}<\tau$, and that $U_{N_{r}}^{k} \neq J_{k}$. Then (since $J_{k}$ is connected) there exists $a \in U_{N_{r}}^{k}, b \in J_{k}-U_{N_{r}}^{k}$ such that $\{a, b\} \in \mathcal{E}(J)$. Let $j=\iota\left(C_{a} \cap C_{b}\right)$. If $S_{r+d}<\tau$, then for some $i \in\{0, \ldots, d-1\}, \bar{\xi}_{S_{r+1}}$ lies in the hyperplane $H_{j}(0)$, so that $A_{N_{r+1}}^{k}(a)=b$. Hence, by (3.21), $p_{1+N_{r+1}}^{k}(b)>0$, so that $b \notin U_{N_{r+d}}^{k}$. So, if $S_{d 2^{d}}<\tau$ we must have $p_{N_{d 2^{d}}}^{k}(a)>0$ for each $a \in J_{k}$. Therefore, by Lemmas 3.16 and 3.18,

$$
p_{N_{m}}(a, b) \geq 4^{-2^{d}} \quad \text { on }\left\{N_{m}<M\right\},
$$

for each $a \in J_{1}, b \in J_{2}$, and any $m \geq d 2^{d}$.

Since each move from $B_{r}$ to $B_{r+1}$ is a corner move of the type considered in Proposition 3.3, we also have from (3.4) that

$$
\mathbb{P}^{x_{k}}\left(T_{N_{m}}<\tau\right) \geq q_{1}^{m}
$$


Let $d 2^{d} \leq m \leq(d+1) 2^{d}$ be such that $\iota\left(B_{m}\right)=\iota\left(L_{f_{1}}\right)$. Choose $b_{k} \in J_{k}$ such that $L_{f_{k}} \subset C_{b_{k}}$. Then note that $X_{T_{N_{m}}}^{k} \in L_{f_{k}}$ on the event $\left\{N_{m}<M, Z_{N_{m}}^{k}=b_{k}\right\}$, so that, using (3.27) and (3.28),

$$
\begin{aligned}
\mathbb{P}\left(T\left(L_{f_{1}}, L_{f_{2}}\right)<\tau\right) & \geq \mathbb{P}\left(X_{T_{N_{m}}}^{k} \in L_{f_{k}}, T_{N_{m}}<\tau\right) \\
& \geq \mathbb{E}\left(p_{N_{m}}\left(b_{1}, b_{2}\right) 1_{\left(N_{m}<M\right)}\right) \\
& \geq 4^{-2^{d}} q_{1}^{(d+1) 2^{d}}
\end{aligned}
$$

which proves (a).

To prove (b), let $S^{\prime}=\inf \left\{t \geq T_{m}: \bar{\xi}_{t} \in M_{i j}\right\}$. Then by Proposition 3.7

$$
\mathbb{P}\left(S^{\prime}<\tau \mid T_{m}<\tau\right) \geq q_{2} .
$$

We have $S^{\prime}=T_{N^{\prime}}$ for some $N^{\prime} \geq m$, on $\left\{S^{\prime}<\tau\right\}$. Then $X_{T_{N^{\prime}}}^{k} \in M_{f_{k} j}$ on the event $\left\{N^{\prime}<M, Z_{N^{\prime}}^{k}=b_{k}\right\}$, and so

$$
\begin{aligned}
\mathbb{P}\left(T\left(M_{f_{1} j}, M_{f_{2} j}\right)<\tau\right) & \geq \mathbb{P}\left(Z_{N^{\prime}}^{k}=b_{k}, k=1,2, N^{\prime}<M\right) \\
& \geq 4^{-2^{d}} \mathbb{P}\left(N^{\prime}<M\right) \\
& =4^{-2^{d}} \mathbb{P}\left(N^{\prime}<M \mid T_{M}<\tau\right) \mathbb{P}\left(T_{m}<\tau\right) \\
& \geq 4^{-2^{d}} q_{2} q_{1}^{m} \geq 4^{-2^{d}} q_{2} q_{1}^{(d+1) 2^{d}},
\end{aligned}
$$

proving (b).

For the next result, we recall from Section 2 the definition of the cubes $D_{n}(x)$ and the stopping times $\sigma_{n}(x)$.

Corollary 3.20 Let $\xi, \eta^{1}, \eta^{2},\left(\Omega, \mathcal{F}, \mathcal{F}_{t}, \mathbb{P}\right)$ be as above, and let $x_{1} \sim x_{2}$, with $x_{k} \notin A_{0}^{*}$. Set $X^{k}=\Gamma_{0}\left(x_{k}, \tilde{F}_{N}, \xi, \eta^{k}\right)$. Let $x_{k} \in A_{k} \in \mathcal{A}$, and let $\left\{\left(A_{1}, A_{2}\right),\left(B_{1}, B_{2}\right)\right\} \in \mathcal{E}\left(\mathcal{A}_{F}^{(2)}\right)$. Then

$$
\mathbb{P}\left(T\left(B_{1}, B_{2}\right)<\sigma_{0}\left(x_{1}, X^{1}\right) \wedge \sigma_{0}\left(x_{2}, X^{2}\right)\right) \geq p_{1} .
$$

Proof This is immediate from (3.17) and Theorem 3.19, by mapping the cubes $D_{0}\left(x_{i}\right)$ to $[-1,1]^{d}$.

Given processes $X_{t}^{1}, X_{t}^{2}$ on $\tilde{F}_{N}$, set for $m \in \mathbb{Z}$,

$$
\kappa_{m}\left(X^{1}, X^{2}\right)=\inf \left\{t \geq 0: X_{t}^{1} \stackrel{m}{\sim} X_{t}^{2}\right\}
$$

Theorem 3.21 Let $N \geq 0$. Let $x_{1} \stackrel{n}{\sim} x_{2}$, with $x_{k} \notin l_{F}^{-n} A_{0}^{*}, x_{k} \in \tilde{F}_{N}$. There exist a pair of process $\left(W_{t}^{1}, W_{t}^{2}\right)$ on $\tilde{F}_{N}$ with $W_{0}^{k}=x_{k}$ such that

(i) $W^{k}$ are $\operatorname{RBM}\left(\tilde{F}_{N}\right), k=1,2$.

(ii) $W_{t}^{1} \stackrel{n}{\sim} W_{t}^{2}$ for $t \geq 0$ 
(iii) Writing $\kappa_{m}=\kappa_{m}\left(W^{1}, W^{2}\right)$ then

$$
\mathbb{P}\left(\sup _{0 \leq t \leq \kappa_{n-1}} \max _{k=1,2}\left\|W_{t}^{k}-x_{k}\right\|_{\infty} \leq\left(1+l_{F}\right) l_{F}^{-n}, \kappa_{n-1}<\infty\right) \geq p_{2}>0
$$

for some $p_{2}=p_{2}\left(d, l_{F}\right)$.

Proof By scaling, it is enough to prove the result for $n=0$. Let $A_{k} \in \mathcal{A}_{F}, C_{k} \in \mathcal{S}_{1}\left(\tilde{F}_{-1}\right)$, $D_{k} \in \mathcal{S}_{-1}^{*}$ be such that $x_{k} \in A_{k} \subset C_{k} \subset D_{k}$. Then there exists $g \in \mathcal{G}\left(D_{1}, D_{2}\right)$ such that $g\left(C_{1}\right)=C_{2}$. Let $x_{1}^{\prime}=g\left(x_{1}\right)$; as $x_{1}^{\prime} \sim x_{1} \sim x_{2}$, we have $x_{1}^{\prime} \sim x_{2}$. Set $A_{1}^{\prime}=g\left(A_{1}\right)$; we also have $A_{1}^{\prime} \sim A_{2}$.

The restriction of the graph $\left(\mathcal{A}_{F}, \mathcal{E}\left(\mathcal{A}_{F}\right)\right)$ to $C_{2}$ is connected, so $A_{1}^{\prime}$ and $A_{2}$ are connected by a chain $A_{1}^{\prime}=\alpha_{0}^{\prime}, \alpha_{1}^{\prime}, \ldots, \alpha_{m}^{\prime}=A_{2}$ where $\alpha_{i}^{\prime} \subset C_{2}, \alpha_{i}^{\prime} \in \mathcal{A}_{F}$, and where $m \leq c_{1}\left(d, l_{F}\right)$. Let $C^{\prime} \in \mathcal{S}_{0}\left(\tilde{F}_{0}\right)$ be such that $A_{2} \subset C^{\prime}$. Then, as in Proposition 3.13, there exists $B_{i}, 0 \leq$ $i \leq m$, such that $B_{i} \subset C^{\prime}, B_{i} \in \mathcal{A}_{F}, B_{0}=B_{m}=A_{2}$, and $\left\{\left(\alpha_{i}^{\prime}, B_{i}\right),\left(\alpha_{i+1}, B_{i+1}\right)\right\} \in \mathcal{E}\left(\mathcal{A}_{F}^{(2)}\right)$ for $0 \leq i \leq m-1$. Set $\alpha_{i}=g^{-1}\left(\alpha_{i}^{\prime}\right)$. Then $\left\{\left(\alpha_{i}, B_{i}\right),\left(\alpha_{i+1}, B_{i+1}\right)\right\} \in \mathcal{E}\left(\mathcal{A}_{F}^{(2)}\right)$ also, and $\left(\alpha_{0}, B_{0}\right)=\left(A_{1}, A_{2}\right),\left(\alpha_{m}, B_{m}\right)=\left(g^{-1}\left(A_{2}\right), A_{2}\right)$, which implies that $\alpha_{m} \stackrel{-1}{\sim} B_{m}$.

Let $\xi, \eta^{k},\left(\mathcal{F}_{t}\right)$ be as in Theorem 3.14, and let $W^{k}=\Gamma_{0}\left(x_{k}, \tilde{F}_{N}, \xi, \eta^{k}\right)$. Set for $i \geq 1$

$$
\begin{gathered}
S_{0}=0, \\
S_{i}=\inf \left\{t \geq S_{i-1}: W_{t}^{1} \in \alpha_{i} \text { and } W_{t}^{2} \in B_{i}\right\}, \\
R_{i}=\inf \left\{t \geq S_{i-1}: W_{t}^{1} \notin D_{0}\left(W_{S_{i-1}}^{1}\right) \text { or } W_{t}^{2} \notin D_{0}\left(W_{S_{i-1}}^{2}\right)\right\} .
\end{gathered}
$$

By Corollary $3.20 \mathbb{P}\left(S_{i}<R_{i} \mid \mathcal{F}_{S_{i-1}}\right) \geq p_{1}$, and therefore if $G=\bigcap_{i=1}^{m}\left\{S_{i}<R_{i}\right\}$ we have $\mathbb{P}(G) \geq p_{1}^{m}$. Note that on the event $G, W_{S_{i}}^{k} \in C_{k}$, so that $\left\|W_{t}^{k}-x_{k}\right\|_{\infty} \leq l_{F}+1$ for $0 \leq t \leq S_{m}$. Write $Y_{k}=W_{S_{m}}^{k}$; on $G$ we have $Y_{2} \in A_{2}, Y_{1} \in \alpha_{m}=g^{-1}\left(A_{2}\right)$, so that $g\left(Y_{1}\right)$ and $Y_{2}$ both lie in $A_{2}$. However $Y_{1} \sim Y_{2}$, and $Y_{1} \stackrel{\sim}{\sim} g\left(Y_{1}\right)$, so that $g\left(Y_{1}\right) \sim Y_{2}$. Hence (since $\left.Y_{k} \notin A_{0}^{*}\right)$ we have $g\left(Y_{1}\right)=Y_{2}$, so that $Y_{1} \stackrel{-1}{\sim} Y_{2}$. Thus $\kappa_{-1} \leq S_{m}$, and taking $p_{2}=p_{1}^{c_{1}\left(d, l_{F}\right)}$ this proves the theorem.

The following result is used to start off the final coupling given in Theorem 3.25.

Lemma 3.22 Let $n \geq 0$, and let $x_{k} \in \tilde{F}_{n}, k=1,2$. There exists a constant $p_{3}=p_{3}(d)>0$ and processes $W_{t}^{k}$ on $\tilde{F}_{n}$ with $W_{0}^{k}=x_{k}$ such that writing $\kappa_{n}=\kappa_{n}\left(W^{1}, W^{2}\right)$,

(i) $W_{t}^{k}$ are $\operatorname{RBM}\left(\tilde{F}_{n}\right), k=1,2$,

(ii) $\mathbb{P}\left(\sup _{0 \leq t \leq \kappa_{n}} \max _{k}\left\|W_{t}^{k}-x_{k}\right\|_{\infty} \leq 2 l_{F}^{-n}, \kappa_{n}<\infty\right) \geq p_{3}$.

Recalling the definition of $\kappa_{n}$ from (3.29), the lemma says that $W_{t}^{1} \stackrel{n}{\sim} W_{t}^{2}$ at time $t=\kappa_{n}$ and neither $W_{t}^{1}$ nor $W_{t}^{2}$ has moved too far from its starting point.

Proof By scaling it is enough to consider the case $n=0$. First we note the following property of a Brownian motion on $\mathbb{R}^{d}$, which is connected with "reflection coupling". Let $B=B(0,1 / 4) \subset \mathbb{R}^{d}$ and let $y_{1}, y_{2} \in B(0,1 / 8)$ with $y_{1}=-y_{2}$. Let $H$ be the hyperplane through 0 perpendicular to the line connecting $y_{1}, y_{2}$, and let $\psi: \mathbb{R}^{d} \rightarrow \mathbb{R}^{d}$ be reflection in 
$H$. Then if $V_{t}^{1}, t \geq 0$, is a Brownian motion on $\mathbb{R}^{d}$ with $V_{0}^{1}=y_{1}, \mathbb{P}\left(\mathcal{T}_{H}\left(V^{1}\right)<\mathcal{T}_{B^{c}}\left(V^{1}\right)\right) \geq$ $c_{1}(d)>0$. (Here $c_{1}$ depends only on the dimension $d$.) So if $V^{2}=\psi\left(V^{1}\right)$, we deduce

$$
\mathbb{P}\left(T_{C}\left(V^{1}, V^{2}\right)<\mathcal{T}_{B^{c}}\left(V^{1}\right) \wedge \mathcal{T}_{B^{c}}\left(V^{2}\right)\right) \geq c_{1}>0 .
$$

Now fix $x_{1}, x_{2} \in \tilde{F}_{0}$, and let $S_{k}^{*} \in \mathcal{S}_{0}^{*}, S_{k} \in \mathcal{S}_{0}\left(\tilde{F}_{0}\right)$ satisfy $x_{k} \in S_{k} \subset S_{k}^{*}$. Let $g \in \mathcal{G}\left(S_{2}^{*}, S_{1}^{*}\right)$ be such that $g: S_{2} \rightarrow S_{1}$. Let $z_{k}$ be the center of $S_{k}$, and write $B_{k}=B\left(z_{k}, 1 / 8\right)$. For $k=1,2$ let $U_{t}^{k}, t \geq 0$ be independent $\operatorname{RBM}\left(\tilde{F}_{0}\right)$ with $U_{0}^{k}=x_{k}$, on a probability space $(\Omega, \mathcal{F}, \mathbb{P})$. Let $\theta_{t}$ be the standard shift operators on $\Omega$ : $U_{s}^{k}\left(\theta_{t} \omega\right)=U_{s+t}^{k}(\omega)$. Let

$$
A_{k}=\left\{U_{1}^{k} \in B_{k}, \sigma\left(x_{k}, U^{k}\right)>1\right\} .
$$

Note that on $A_{k}$ we have $\sup _{0 \leq s \leq 1}\left\|U_{s}^{k}-x_{k}\right\|_{\infty} \leq 3 / 2$. By Proposition 2.6 there exists $c_{2}=c_{2}(d)>0$, (not depending on $x_{k}$ ), such that

$$
\mathbb{P}^{x_{k}}\left(A_{k}\right) \geq c_{2}
$$

Let $Y_{1}=U_{1}^{1}, Y_{2}=g\left(U_{1}^{2}\right)$. Set $Z=\left(Y_{1}+Y_{2}\right) / 2$, and let $B^{\prime}=B(Z, 1 / 4)$. On $A_{1} \cap A_{2}$ we have $\left|Y_{k}-z_{1}\right| \leq 1 / 8$, so that $\left|Z-z_{1}\right| \leq 1 / 8$, and $\left|Z-Y_{k}\right| \leq 1 / 8$. In particular $B^{\prime} \subset S_{1}$. Let $H$ be the hyperplane containing $Z$ and perpendicular to the direction $Y_{2}-Y_{1}$, and let $h: \mathbb{R}^{d} \rightarrow \mathbb{R}^{d}$ be reflection in $H$. Set $W^{1}=U^{1}$, and let $\tilde{U}_{t}^{2}=h\left(U_{t}^{1}\right), t \geq 0$. Set

$$
W_{t}^{2}=U_{t}^{2} 1_{\left(A_{1} \cap A_{2}\right)^{c}}+1_{A_{1} \cap A_{2}}\left[1_{[0,1]}(t) U_{t}^{2}+1_{(1, \infty)}(t) g^{-1}\left(\tilde{U}_{t}^{2}\right)\right] .
$$

If $S=\inf \left\{t \geq 1:\left(W_{t}^{1}, W_{t}^{2}\right) \notin S_{1} \times S_{2}\right\}$ then $W^{2}$ is a $\operatorname{RBM}\left(\tilde{F}_{0}\right)$ started at $x_{2}$ for $0 \leq t \leq S$. It is then straightforward to extend $W^{2}$ to a $\operatorname{RBM}\left(\tilde{F}_{0}\right)$ on $[0, \infty)$.

Write $T_{C}^{\prime}=T_{C}\left(U^{1}, \tilde{U}^{2}\right) \circ \theta_{1}, \tau^{\prime}=\left(\tau_{B^{\prime}}\left(U^{1}\right) \wedge \tau_{B^{\prime}}\left(U^{2}\right)\right) \circ \theta_{1}$, and let $A_{3}=\left\{T_{C}^{\prime}<\tau^{\prime}\right\}$. By (3.31) we have

$$
\mathbb{P}\left(T_{C}^{\prime}<\tau^{\prime} \mid A_{1} \cap A_{2}\right) \geq c_{1},
$$

so that, writing $A=A_{1} \cap A_{2} \cap A_{3}, \mathbb{P}(A) \geq c_{1} c_{2}^{2}$. On $A$ we have, by the definition of $W^{2}$, that $W_{T_{C}^{\prime}}^{1} \sim W_{T_{C}^{\prime}}^{2}$, so that $\kappa_{0} \leq T_{C}^{\prime}$. Also, on $A, \max _{k} \sup _{1 \leq t \leq T_{C}^{\prime}}\left\|W_{t}^{k}-W_{1}^{k}\right\|_{\infty} \leq 1 / 4$, so that $\max _{k} \sup _{0 \leq t \leq T_{C}^{\prime}}\left\|W_{t}^{k}-x_{k}\right\|_{\infty} \leq 3 / 2+1 / 4<2$.

Recall from (2.6) the definition of the exit times $\sigma_{r}(x)$.

Proposition 3.23 Let $N, m, n \in \mathbb{Z}, m \geq n+3, x \in \tilde{F}_{N}$, and $z$ be the center of $D_{n}(x)$. Suppose $x_{1} \stackrel{m}{\sim} x_{2}, x_{k} \notin A_{0}^{*}$, and $\left\|x_{k}-z\right\|_{\infty} \leq l_{F}^{-n}\left(1-l_{F}^{-1}\right), k=1,2$. Then there exist $\operatorname{RBM}\left(\tilde{F}_{N}\right) W_{t}^{k}$, with $W_{0}^{k}=x_{k}$, such that if $n_{0}=d 2^{d}\left(2 l_{F}\right)^{(m-n) d}$, then

$$
\mathbb{P}\left(T_{C}\left(W^{1}, W^{2}\right)<\sigma_{n}\left(z, W^{1}\right) \wedge \sigma_{n}\left(z, W^{2}\right)\right) \geq p_{1}^{n_{0}} .
$$

Proof Suppose first that $m=0$. (So $|n|=-n \geq 3$.) Let $x_{k} \in A_{k} \in \mathcal{A}_{F}$, and let $C_{k} \in \mathcal{S}_{0}\left(\tilde{F}_{0}\right)$ be such that $A_{k} \subset C_{k}$. Note that in the $\|\cdot\|_{\infty}$ norm $C_{k}$ is at least a distance $l_{F}^{|n|-1}-1 \geq$ $l_{F}^{2}-1 \geq l_{F}+1$, from $D_{n}(x)^{c}$. Hypotheses $2.1(\mathrm{H} 1)-(\mathrm{H} 4)$ imply we can find a chain of cubes in $\mathcal{S}_{0}\left(\tilde{F}_{0}\right)$ of length less than or equal to $n_{1}=\left(2 l_{F}\right)^{|n| d}$ connecting $C_{1}$ and $C_{2}$, and contained in the cube center $z$ and side $2\left(l_{F}^{|n|}-2\right)$. If $A, B \subset C$, where $C \in \mathcal{S}_{0}\left(\tilde{F}_{0}\right)$, then 
certainly $d_{F}(A, B) \leq d 2^{d}$, the total number of half-faces contained in $C$. So we can find a chain $A_{1}=\alpha_{0}, \alpha_{1}, \ldots, \alpha_{r}=A_{2}$ in $\mathcal{A}_{F}$, where $r \leq n_{0}=d 2^{d}\left(2 l_{F}\right)^{-n d}$, and where each $\alpha_{i}$ is a distance at least 2 from $D_{n}(x)^{c}$.

We have $A_{1} \sim A_{2}$; we can therefore as in Proposition 3.13 find a chain $\left(\alpha_{i}, B_{i}\right)$ in $\mathcal{A}_{F}^{(2)}$ such that $\left(\alpha_{0}, B_{0}\right)=\left(A_{1}, A_{2}\right),\left(\alpha_{r}, B_{r}\right)=\left(A_{2}, A_{2}\right)$, and each $B_{i} \subset C_{2}$. Using Corollary 3.20, we therefore have that if $W^{k}=\Gamma_{0}\left(x_{k}, \tilde{F}_{N}, \xi, \eta^{k}\right)$, with $\xi, \eta^{k}$ as above, then the process $\left(W^{1}, W^{2}\right)$ moves along the chain $\left(\alpha_{i}, B_{i}\right)$ with probability at least $p_{1}^{r} \geq p_{1}^{n_{0}}$. As the half-faces in the chain are all a distance at least 2 from $D_{n}(x)^{c}$, and since $C_{2} \subset D_{0}\left(x_{2}\right) \subset$ $D_{n+3}\left(x_{2}\right)$, it follows that

$$
\mathbb{P}\left(T_{D_{n+3}\left(x_{2}\right)}\left(W^{1}\right)<\sigma_{n}(z)\left(W^{1}\right), T_{C}\left(W^{1}, W^{2}\right)<\sigma_{0}\left(z, W^{1}\right) \wedge \sigma_{0}\left(z, W^{2}\right)\right) \geq p_{1}^{n_{0}} .
$$

This implies the result when $m=0$; if $m \neq 0$, we can scale by $l_{F}^{m}$, and replace $(N, m, n)$ by $(N-m, 0, n-m)$.

The following lower bound on the probability of hitting small cubes is an essential ingredient in the Harnack inequality Theorem 4.3.

Corollary 3.24 Let $N, n \in \mathbb{Z}, x \in \tilde{F}_{N}$, and $z$ be the center of $D_{n}(x)$. There exists $\delta=\delta\left(l_{F}, d\right)$ such that if $y_{1}, y_{2} \in B\left(z, \frac{1}{2} l_{F}^{-n}\right)$, and $W$ is a $\operatorname{RBM}\left(\tilde{F}_{N}\right)$, then

$$
\mathbb{P}^{y_{1}}\left(\sigma_{n+j+2}\left(y_{2}, W\right)<\sigma_{n}(z)\right) \geq \delta^{j}, \quad j \geq 1 .
$$

Proof We begin with the case $j=1$. Take $m=n+3$; as in the previous proof we can suppose $m=0$. Let $x_{1}=y_{1}$, and choose $x_{2} \in D_{0}\left(y_{2}\right)$ such that $x_{1} \sim x_{2}$, and $C_{2}$ so that $C_{2} \subset D_{0}\left(y_{2}\right)$. Then (3.32) implies that, writing $n_{1}=d 2^{d}\left(2 l_{F}\right)^{3 d}, \delta=p_{1}^{n_{1}}$,

$$
\mathbb{P}\left(\sigma_{0}\left(y_{2}\right)<\sigma_{n}(z)\right) \geq \delta .
$$

We now iterate. Write $S_{k}=\sigma_{n+k+2}\left(y_{2}\right)$, and let $z_{k}$ be the center of $D_{k}\left(y_{2}\right)$. Since $W_{S_{1}}$, $y_{2} \in B_{\infty}\left(z_{n+1}, l_{F}^{-n-1}\right)$, and $\sigma_{n+1}\left(z_{n+1}\right) \leq \sigma_{n}\left(z_{n}\right)$, we have

$$
\mathbb{P}^{y_{1}}\left(S_{2}<\sigma_{n}(z)\right) \geq \mathbb{E}^{y_{1}}\left(1_{\left(S_{1}<\sigma_{n}(z)\right)} \mathbb{P}^{W_{S_{1}}}\left(S_{2}<\sigma_{n+1}\left(z_{n+1}\right)\right)\right) \geq \delta^{2} .
$$

This gives the case $j=2$, and by induction we obtain the general case.

Theorem 3.25 Let $N, n \in \mathbb{Z}, x_{0}, x, y \in \tilde{F}_{N}, \varepsilon>0$. There exist constants $p_{4}=p_{4}\left(d, l_{F}\right)>0$, $\delta=\delta\left(\varepsilon, d, l_{F}\right)>0$, and coupled $\operatorname{RBM}\left(\tilde{F}_{N}\right) W_{t}^{x}, W_{t}^{y}$, with $W_{0}^{x}=x, W_{0}^{y}=y$, with the following properties.

(a) If $x, y \in D_{n+2}\left(x_{0}\right)$, then

$$
\mathbb{P}\left(T_{C}\left(W^{x}, W^{y}\right)<\sigma_{n}\left(x, W^{x}\right) \wedge \sigma_{n}\left(x, W^{y}\right)\right)>p_{4} .
$$

(b) If $\|x-y\|_{\infty} \leq l_{F}^{-n} \delta$, then

$$
\mathbb{P}\left(T_{C}\left(W^{x}, W^{y}\right)<\sigma_{n}\left(x, W^{x}\right) \wedge \sigma_{n}\left(x, W^{y}\right)\right)>1-\varepsilon .
$$


Proof By scaling it is sufficient to prove this for $n=0$.

Set $A_{\mathbb{Z}}^{*}=\bigcup_{n \in \mathbb{Z}} l_{F}^{-n} A_{0}^{*}$, and note that $A_{\mathbb{Z}}^{*}$ is polar for any $\operatorname{RBM}\left(\tilde{F}_{N}\right)$; this ensures that the various sequences of successive disjoint hits below are well-defined. Write $x_{1}=x, x_{2}=y$. Let $m_{0} \geq 0$ (to be chosen below), and let $M \geq \max \left(N, m_{0}\right)$. We construct processes $W_{t}^{k}$ successively on the intervals $\left[0, \kappa_{M}\right],\left[\kappa_{M}, \kappa_{M-1}\right], \ldots$, where the $\kappa$ are given by (3.29), as follows.

Suppose first that $W_{t}^{k}, 0 \leq t \leq \kappa_{m}=\kappa_{m}\left(W^{1}, W^{2}\right), k=1,2$, have been defined. Use Theorem 3.21 applied conditionally on $\sigma\left(W_{s}^{k}, s \leq \kappa_{m}, k=1,2\right)$ to define $\operatorname{RBM}\left(\tilde{F}_{N}\right) X_{t}^{k, m, 1}$, $t \geq 0$, such that

$$
\begin{aligned}
& X_{0}^{k, m, 1}=W_{\kappa_{m}}^{k}, \quad k=1,2, \\
& X_{t}^{1, m, 1} \stackrel{m}{\sim} X_{t}^{2, m, 1}, \quad t \geq 0,
\end{aligned}
$$

and the estimate (3.30) holds. Set

$$
S_{m, 1}=\inf \left\{t \geq 0: \max _{k}\left\|X_{t}^{k, m, 1}-W_{\kappa_{m}}^{k}\right\|_{\infty} \geq\left(1+l_{F}\right) l_{F}^{-m}\right\} .
$$

If $S_{m, 1}>\kappa_{m-1}\left(X^{1, m, 1}, X^{2, m, 1}\right)$ we say that "trial $(m, 1)$ succeeds", and otherwise we say "trial $(m, 1)$ fails"; (3.30) implies the probability of success is at least $p_{2}$. We now define $W^{k}$ on an additional interval in $[0, \infty)$ by

$$
W_{\kappa_{m}+t}^{k}=X_{t}^{k, m, 1}, \quad 0 \leq t \leq S_{m, 1} \wedge \kappa_{m-1}\left(X^{1, m, 1}, X^{2, m, 1}\right)
$$

If trial $(m, 1)$ succeeds we have defined $W^{k}$ on $\left[0, \kappa_{m-1}\right]$, and are ready to repeat the same construction at level $m-1$. If trial $(m, 1)$ fails, we repeat the attempt, using Theorem 3.21 to define $\operatorname{RBM}\left(\tilde{F}_{N}\right), X_{t}^{k, m, 2}, t \geq 0$, satisfying (3.34), (3.30), and with initial conditions

$$
X_{0}^{k, m, 2}=X_{S_{m, 1}}^{k, m, 1}, \quad k=1,2 .
$$

Continuing in this way, we make trials $(m, i), i=1,2, \ldots$ at achieving coupling at level $m-1$, until one succeeds. As the probability of success (conditional on the past) is at least $p_{2}$ for each trial, we have $\kappa_{m-1}<\infty$ a.s.

We use a similar argument to define $W^{k}$ on the initial segment $\left[0, \kappa_{M}\right]$, except that here we use Lemma 3.22 instead of Theorem 3.21. Finally, we note that $T_{C}\left(W^{1}, W^{2}\right)=\kappa_{n}$ for all sufficiently small $n$, and define $W^{k}$ on $\left[T_{C}\left(W^{1}, W^{2}\right), \infty\right)$ by taking $W_{t}^{1}$ to be a $\operatorname{RBM}\left(\tilde{F}_{n}\right)$ for $t \geq T_{C}\left(W^{1}, W^{2}\right)$, and setting $W_{t}^{2}=W_{t}^{1}$.

Set $p=\min \left(p_{2}, p_{3}\right)$, and let $a_{0}, a_{1}, \ldots, a_{M}, \ldots$ be positive integers, to be chosen below. Let $Y_{i}, 0 \leq i \leq M+1$, be the number of the trial at level $i$ that first succeeds. From (3.30) we have

$$
\mathbb{P}\left(Y_{i}>r \mid Y_{i+1}, \ldots, Y_{M+1}\right)<(1-p)^{r}
$$

Let $m_{0} \geq 0$, and let

$$
G=\left\{Y_{i} \leq a_{i}, m_{0} \leq i \leq M+1\right\}
$$

Therefore

$$
\mathbb{P}\left(G^{c}\right) \leq \sum_{i=m_{0}}^{M+1}(1-p)^{a_{i}}
$$


while on $G$ we have

$$
\left\|V_{t}^{k}-x_{k}\right\|_{\infty} \leq \sum_{i=m_{0}}^{M+1} a_{i}\left(1+l_{F}\right) l_{F}^{-i}, \quad \text { for } 0 \leq t \leq \kappa_{m_{0}} .
$$

Now take $a_{i}=\left(2 l_{F} / 3\right)^{i}$; then as $l_{F} \geq 3$ we have $a_{i} \geq i$, so that

$$
\mathbb{P}\left(G^{c}\right) \leq p^{-1}(1-p)^{m_{0}}
$$

while

$$
\sum_{i=m_{0}}^{\infty} a_{i}\left(1+l_{F}\right) l_{F}^{-i} \leq 3\left(1+l_{F}\right)(2 / 3)^{m_{0}} .
$$

Now choose $m_{0}$ to be the smallest integer such that $m_{0} \geq 3,3\left(1+l_{F}\right) l_{F}^{-m_{0}} \leq\left(2 l_{F}\right)^{-1}$, and $p^{-1}(1-p)^{m_{0}} \leq 1 / 2$. Note that $m_{0}$ depends only on $d$ and $l_{F}$. Let $z$ be the center of the cube $D_{0}(x)$. On $G$ we have, for $k=1,2$,

$$
\left\|x_{k}-z\right\|_{\infty} \leq\left\|x_{0}-z\right\|_{\infty}+\left\|x_{k}-x_{0}\right\|_{\infty} \leq \frac{1}{2}+\frac{3}{2} l_{F}^{-2} \leq \frac{2}{3}
$$

Therefore

$$
\sup _{0 \leq t \leq \kappa_{m_{0}}}\left\|V_{t}^{k}-z\right\|_{\infty} \leq\left\|x_{k}-z\right\|_{\infty}+\left(2 l_{F}\right)^{-1} \leq \frac{2}{3}+\left(2 l_{F}\right)^{-1} \leq 1-l_{F}^{-1}
$$

so that on $G, \kappa_{m_{0}}<\sigma\left(z, V^{1}\right) \wedge \sigma\left(z, V^{2}\right)$.

By Proposition 3.23 there exist $\operatorname{RBM}\left(\tilde{F}_{N}\right) U_{t}^{k}$, with $U_{0}^{k}=V_{\kappa_{m_{0}}}^{k}$, such that

$$
\mathbb{P}\left(T_{C}\left(U^{1}, U^{2}\right)<\sigma_{0}\left(z, U^{1}\right) \wedge \sigma_{0}\left(z, U^{2}\right)\right) \geq p_{1}^{n_{1}}
$$

where $n_{1}=d 2^{d}\left(2 l_{F}\right)^{m_{0} d}$. Now define

$$
W_{t}^{k}= \begin{cases}V_{t}^{k} & 0 \leq t \leq \kappa_{m_{0}} \\ U_{t-\kappa_{m_{0}}}^{k} & \kappa_{m_{0}} \leq t\end{cases}
$$

and let $W_{t}^{x}=W_{t}^{1}, W_{t}^{y}=W_{t}^{y} 1_{\left(t<T_{C}\right)}+W_{t}^{x} 1_{\left(t \geq T_{C}\right)}$. We have

$$
\begin{aligned}
& \mathbb{P}\left(T_{C}\left(W^{x}, W^{y}\right)<\sigma_{0}\left(x, W^{x}\right) \wedge \sigma_{0}\left(x, W^{y}\right)\right) \\
& \quad \geq \mathbb{P}(G) \mathbb{P}\left(T_{C}\left(U^{1}, U^{2}\right)<\sigma_{0}\left(z, U^{1}\right) \wedge \sigma_{0}\left(z, U^{2}\right) \mid G\right) \\
& \quad \geq \frac{1}{2} p_{1}^{n_{1}}=p_{4} .
\end{aligned}
$$

Since $p_{4}$ depends only on $d$ and $l_{F}$, this proves (a).

To prove (b), we use (a) iteratively. Let $m$ be the smallest integer divisible by 4 such that $\left(1-p_{4}\right)^{(m-5) / 4}<\varepsilon$, and let $\delta=\frac{1}{2} l_{F}^{-m}$. We define inductively $\operatorname{RBM}\left(\tilde{F}_{N}\right) W^{x}, W^{y}$ 
successively on intervals $\left[T_{i}, T_{i+1}\right]$, stopping times $T_{i}$, and random variables $X_{i}=W_{T_{i}}^{x}$, $Y_{i}=W_{T_{i}}^{y}$ such that $Y_{i} \in D_{m-4 i}\left(X_{i}\right)$.

We take $X_{0}=x, Y_{0}=y$; since $\|x-y\|_{\infty} \leq \delta \leq \frac{1}{2} l_{F}^{-m}$, we have $y \in D_{m}(x)$. Suppose now $W^{x}, W^{y}$ are defined on $\left[0, T_{i}\right]$, and $Y_{i} \in D_{m-4 i}\left(X_{i}\right)$. Apply (a) with $n=m-4 i-2$ to obtain $\operatorname{RBM}\left(\tilde{F}_{N}\right) V_{t}^{x, i}, V_{t}^{y, i}$ with $V_{0}^{x, i}=X_{i}, V_{0}^{y, i}=Y_{i}$ satisfying (3.33). Set $S_{i}=\sigma_{m-4 i-2}\left(X_{i}, V^{x, i}\right) \wedge$ $\sigma_{m-4 i-2}\left(X_{i}, V^{y, i}\right)$ and $T_{C}^{i}=T_{C}\left(V^{x, i}, V^{y, i}\right)$, and let

$$
\begin{gathered}
T_{i+1}=T_{i}+S_{i}, \\
W_{t}^{z}=V_{t-T_{i}}^{z, i}, \quad T_{i} \leq t \leq T_{i+1}, \quad z=x, y, \\
X_{i+1}=W_{T_{i+1}}^{x}, \quad Y_{i+1}=W_{T_{i+1}}^{y} .
\end{gathered}
$$

Note that as $V_{t}^{x, i}=V_{t}^{y, i}$ for $t>T_{C}^{i}$, we have $W_{t}^{x}=W_{t}^{y}$ for $t>T_{C}=T_{C}\left(W^{x}, W^{y}\right)$. If $T_{C}^{i}<$ $S_{i}$ then $Y_{i+1}=X_{i+1} \in D_{m-4 i-4}\left(X_{i+1}\right)$. Otherwise we have, as $X_{i+1}, Y_{i+1} \in D_{m-4 i-2}\left(X_{i}\right)$, that

$$
\begin{gathered}
\left\|X_{i+1}-X_{i}\right\|_{\infty} \vee\left\|Y_{i+1}-Y_{i}\right\|_{\infty} \leq \frac{3}{2} l_{F}^{-(m-4 i-2)}, \\
\left\|X_{i+1}-Y_{i+1}\right\|_{\infty} \leq 2 l_{F}^{-(m-4 i-2)} \leq \frac{1}{2} 4 l_{F}^{-2} l_{F}^{-(m-4 i-4)},
\end{gathered}
$$

so since $4 l_{F}^{-2}<1$ we have $Y_{i+1} \in D_{m-4(i+1)}\left(X_{i+1}\right)$.

For each $i$, we have, by (a)

$$
\mathbb{P}\left(T_{0}^{i}<S_{i} \mid \mathcal{F}_{T_{i}}\right) \geq p_{4}
$$

Let $H=\left\{T_{C}^{i}<S_{i}\right.$ for some $\left.i \leq \frac{1}{4}(m-5)\right\}$, so that $\mathbb{P P}^{\prime}\left(H^{c}\right) \leq\left(1-p_{4}\right)^{(m-5) / 4}<\varepsilon$. On $H$ we have, using (3.35), and writing $l=(m-5) / 4$,

$$
\begin{aligned}
\left\|X_{l}-x\right\|_{\infty} \vee\left\|Y_{l}-y\right\|_{\infty} & \leq \sum_{i=0}^{l} \frac{3}{2} l_{F}^{-m+4 i+2} \\
& \leq \frac{3}{2} l_{F}^{2-m} l_{F}^{4(l+1)}\left(l_{F}^{4}-1\right)^{-1} \\
& \leq \frac{3}{2} l_{F}^{2-m} l_{F}^{-3} l_{F}^{m-5+4}=\frac{3}{2} l_{F}^{2}<\frac{1}{6}
\end{aligned}
$$

So, on $H, W^{x}$ and $W^{y}$ couple before leaving $D_{0}(x)$, and $\mathbb{P}(H) \geq 1-\varepsilon$.

Remark 3.26 Much of the argument above only uses the symmetry of $F_{1}$ with respect to reflection in the sets $H_{i}(1 / 2)$, which is a strictly weaker condition than Hypothesis 2.1 (H1). We do use (H1) however in the proof of Proposition 3.5, when we reflect in the hyperplanes $H_{i j}$.

Remark 3.27 The arguments above use essentially only three properties of the Markov process $W$ : its continuity, the fact that $W$ is symmetric with respect to the symmetries of the cube, and the fact that $A_{0}^{*}$ is polar for $W$. We use this in [BB6], where we couple a "cable process" associated with the graphical pre-carpet. 


\section{Uniform Harnack Inequality}

In this section we use the coupling of Section 3 to prove a Harnack inequality for harmonic functions on $\tilde{F}_{N}$ with constants which are independent of $N$. The proofs use ideas of [LR] and $[\mathrm{Ca}]$. For a function $f$ and a Borel set $A$, define

$$
\operatorname{Osc}_{A} f=\sup _{A} f-\inf _{A} f
$$

Proposition 4.1 There exists $\theta \in(0,1)$ independent of $n$ and $N$ such that if $x_{0} \in \tilde{F}_{N}$, and $h$ is harmonic on $D_{n}\left(x_{0}\right) \cap \tilde{F}_{N}$, then

$$
\operatorname{Osc}_{D_{n+2}\left(x_{0}\right)} h \leq \theta \operatorname{Osc}_{D_{n}\left(x_{0}\right)} h
$$

Proof There is nothing to prove if $\mathrm{Osc}_{D_{n}\left(x_{0}\right)} h$ is infinite, so we may suppose $h$ is bounded on $D_{n}\left(x_{0}\right)$. By adding a constant to $h$, we may suppose $\inf _{D_{n}\left(x_{0}\right)} h=0$. Let $x, y \in D_{n+2}\left(x_{0}\right) \cap$ $\tilde{F}_{N}$, and let $W^{x}, W^{y}$ be the $\operatorname{RBM}\left(\tilde{F}_{N}\right)$ given in Theorem 3.25(a). Let $U=\sigma_{n}\left(x_{0}, W^{x}\right) \wedge$ $\sigma_{n}\left(x_{0}, W^{y}\right)$ and $T_{C}=T_{C}\left(W^{x}, W^{y}\right)$; by (3.33) we have

$$
\mathbb{P}\left(T_{C} \geq U\right) \leq 1-p_{4},
$$

where $p_{4}$ depends only on $d$ and $l_{F}$. Set $\theta=1-p_{4}$. Since $h$ is harmonic we have

$$
\begin{aligned}
h(x) & =\mathbb{E} h\left(W_{U \wedge T_{C}}^{x}\right) \\
& =\mathbb{E}\left[h\left(W_{T_{C}}^{x}\right) ; T_{C}<U\right]+\mathbb{E}\left[h\left(W_{U}^{x}\right) ; T_{C} \geq U\right]
\end{aligned}
$$

and similarly

$$
h(y)=\mathbb{E}\left[h\left(W_{T_{C}}^{y}\right) ; T_{C}<U\right]+\mathbb{E}\left[h\left(W_{U}^{y}\right) ; T_{C} \geq U\right] .
$$

Since $h\left(W_{T_{C}}^{x}\right)=h\left(W_{T_{C}}^{y}\right)$, subtracting (4.2) from (4.1) gives

$$
\begin{aligned}
|h(x)-h(y)| & =\left|\mathbb{E}\left[h\left(W_{U}^{x}\right)-h\left(W_{U}^{y}\right) ; T_{C} \geq U\right]\right| \\
& \leq \mathbb{P}\left(T_{C} \geq U\right) \operatorname{Osc}_{D_{n}\left(x_{0}\right)} h \\
& \leq \theta \operatorname{Osc}_{D_{n}\left(x_{0}\right)} h .
\end{aligned}
$$

Theorem 4.2 There exist $M$ and $\alpha$ depending only on $d$ and $l_{F}$ such that if $x_{0} \in \tilde{F}_{n}$ and $h$ is harmonic in $D_{n}\left(x_{0}\right) \cap \tilde{F}_{N}$, then

$$
|h(x)-h(y)| \leq M|x-y|^{\alpha} l_{F}^{n \alpha} \sup _{D_{n}\left(x_{0}\right)}|h|, \quad x, y \in D_{n+2}\left(x_{0}\right)
$$

Proof By iterating Proposition 4.1,

$$
\operatorname{Osc}_{D_{n+2 j}(x)} h \leq \theta \operatorname{Osc}_{D_{n+2(j-1)}(x)} h \leq \theta^{j} \operatorname{Osc}_{D_{n}(x)}|h| \leq 2 \theta^{j} \sup _{D_{n}\left(x_{0}\right)}|h| .
$$


Let $\|x-y\|_{\infty}=r$, and choose $j$ such that $y \in D_{n+2 j}(x)-D_{n+2(j+1)}(x)$; by Lemma 2.3

$$
\frac{3}{2} l_{F}^{-n-2 j} \geq r \geq \frac{1}{2} l_{F}^{-n-2 j-2} .
$$

So $c_{1} \theta^{j} \geq\left(l_{F}^{n} r\right)^{\log (1 / \theta) / 2 \log l_{F}} \geq c_{2} \theta^{j}$, and hence by (4.3),

$$
|h(y)-h(x)| \leq \operatorname{Osc}_{D_{n+2 j}(x)} h \leq c_{3} l_{F}^{n \alpha} r^{\alpha} \sup _{D_{n}\left(x_{0}\right)}|h|,
$$

where $\alpha=\log (1 / \theta) / 2 \log l_{F}$.

Theorem 4.3 (Uniform Harnack Inequality) There exists $c_{1}$ depending only on $d$ and $l_{F}$ such that if $x_{0} \in \tilde{F}_{N}$ and $h$ is nonnegative and harmonic in $D_{n}\left(x_{0}\right) \cap \tilde{F}_{N}$, then

$$
h(x) \leq c_{1} h(y), \quad x, y \in D_{n+4}\left(x_{0}\right) .
$$

Proof Let $z$ be the center of $D_{n}\left(x_{0}\right)$. By looking at $h+\varepsilon$ and then letting $\varepsilon \downarrow 0$, we may assume $h$ is bounded below by a positive constant in $D_{n}\left(x_{0}\right)$. Multiplying by a constant, we may assume $\inf _{D_{n+4}\left(x_{0}\right)} h=1$. Since $\tilde{F}_{N}$ is a Lipschitz domain, $h$ is bounded and continuous on $A=B\left(z, l_{F}^{-n}\left(1-l_{F}^{-1}\right)\right)$; we need to show that we can bound $h$ in $D_{n+4}\left(x_{0}\right)$ by a constant independent of $n$ and $N$.

By Corollary 3.24 we have, for $x \in A$, and $\delta=\delta\left(d, l_{F}\right)$,

$$
\mathbb{P}^{y}\left(\mathcal{T}_{D_{n+j}(x)}<\sigma_{n}\left(x_{0}\right)\right)>\delta^{j}, \quad j \geq 3 .
$$

This yields an estimate on the minimum of $h$ on $D_{n+j}(x)$; we have

$$
1=h(y) \geq \mathbb{E}^{y}\left[h\left(X\left(\mathcal{T}_{D_{n+j}(x)}\right)\right) ; \mathcal{T}_{D_{n+j}(x)}<\sigma_{n}\left(x_{0}\right)\right] \geq \delta^{j} \inf _{D_{n+j}(x)} h,
$$

so that

$$
\inf _{D_{n+j}(x)} h \leq \delta^{-j}, \quad x \in A, j \geq 3 .
$$

Now choose $M \geq 3$ so that $\theta^{-M} \geq \delta^{-2} /\left(\delta^{-1}-1\right)$. Let $j_{0}=2 M-2$. If $x \in A, D_{n+j-2 M}(x) \subset$ $D_{n}\left(x_{0}\right)$ for $j \geq j_{0}$, and so from Proposition 4.1

$$
\begin{aligned}
\operatorname{Osc}_{D_{n+j-2 M}(x)} h & \geq \theta^{-M} \operatorname{Osc}_{D_{n+j}(x)} h \\
& \geq \frac{\delta^{-2}}{\delta^{-1}-1} \operatorname{Osc}_{D_{n+j}(x)} h, \quad j \geq j_{0}, x \in A .
\end{aligned}
$$

Let $K=2 M+3$. Suppose there exists $x^{\prime} \in D_{n+4}\left(x_{0}\right)$ such that $h\left(x^{\prime}\right) \geq \delta^{-K-j_{0}-2}$. We use induction to construct a sequence $x_{j}, j \geq j_{0}$ such that $x_{j_{0}}=x^{\prime}$ and

$$
\begin{gathered}
h\left(x_{j}\right) \geq \delta^{-K-j-1}, \\
\left\|x_{j}-z\right\|_{\infty} \leq \frac{3}{2} l_{F}^{-n-4}+\frac{3}{2} l_{F}^{-n-3} \sum_{i=j_{0}}^{j} l_{F}^{-j} .
\end{gathered}
$$


Suppose we have constructed $x_{j_{0}}, \ldots, x_{j}$. Since by (4.8) $x_{j} \in A$, we have by (4.5) that

$$
\inf _{D_{n+K+j}\left(x_{j}\right)} h \leq \delta^{-K-j}
$$

while by (4.7)

$$
h\left(x_{j}\right) \geq \delta^{-K-j-1} .
$$

Hence

$$
\operatorname{Osc}_{D_{n+K+j}\left(x_{j}\right)} h \geq\left(\delta^{-1}-1\right) \delta^{-K-j} .
$$

By (4.6),

$$
\operatorname{Osc}_{D_{n+K+j-2 M}\left(x_{j}\right)} h \geq \delta^{-K-j-2} .
$$

Since $h$ is nonnegative, this implies that there must exist a point $x_{j+1} \in D_{n+j+3}\left(x_{j}\right)$ such that $h\left(x_{j+1}\right) \geq \delta^{-K-j-2}$. As $\left\|x_{j+1}-x_{j}\right\|_{\infty} \leq \frac{3}{2} l_{F}^{-n-j-3}, x_{j+1}$ satisfies (4.7) and (4.8).

By induction, we therefore have a sequence $x_{j}, j \geq j_{0}$ in $A$ with $h\left(x_{j}\right) \rightarrow \infty$, which contradicts the fact that $h$ is bounded on $A$. It follows that $h$ is bounded on $D_{n+4}\left(x_{0}\right)$ by $\delta^{-K-j_{0}-2}$.

Remark 4.4 Note that the proofs of the results in this section do not use the symmetry assumption Hypothesis 2.1 (H1) directly, but only through Corollary 3.24 and Theorem 3.25. So if $\tilde{F}_{N}$ is a generalized Sierpinski carpet at level $N$, for which $F_{1}$ satisfies Hypothesis 2.1 (H2)-(H4), and in addition we know that Corollary 3.24 and Theorem 3.25 hold for $\tilde{F}_{N}$, then all the results in this section hold for $\tilde{F}_{N}$.

Remark 4.5 The proof of Theorem 1.1. is immediate from Theorem 4.3.

\section{Constants and Inequalities}

\section{Constants}

We define the resistance constant $R_{n}$ by

$$
R_{n}^{-1}=\inf \left\{\int_{l_{F}^{n} F_{n}}|\nabla f|^{2} d x: f=0 \text { on } x_{1}=0, f=1 \text { on } x_{1}=l_{F}^{n}\right\} .
$$

Thus $R_{n}$ is the resistance between two opposite faces of the set $l_{F}^{n} F_{n}$. In [McG] (see also [KZ] and for the case $d=2[\mathrm{BB} 3])$, it is shown that there exists a constant $\rho_{F}$ and constants $c_{1}$, $c_{2}$ such that

$$
c_{1} \rho_{F}^{n} \leq R_{n} \leq c_{2} \rho_{F}^{n} .
$$

The proof uses a subadditivity argument, which does not yield the value of $\rho_{F}$. We call $\rho_{F}$ the resistance scale factor of $F$, and define the time scale factor by $t_{F}=\left(m_{F}\right)\left(\rho_{F}\right)$. We define the fractal dimension, dimension of the walk, and spectral dimension of $F$ by

$$
\begin{gathered}
d_{f}=\log m_{F} / \log l_{F}, \\
d_{w}=\log t_{f} / \log l_{F}, \\
d_{s}=2 d_{f} / d_{w}=2 \log m_{F} / \log t_{F} .
\end{gathered}
$$


$d_{f}$ is the Hausdorff dimension (and also the packing dimension ) of $F$ and $\tilde{F}$.

Using standard shorting and cutting arguments (see [DS], and also [BB3, Prop. 5.3]) we have the following estimates for $\rho_{F}$.

\section{Proposition 5.1}

(a) $t_{F} \geq l_{F}^{2}$.

(b) $\rho_{F} \leq 2^{1-d} l_{F}$.

Proof (a) Note that $l_{F}^{n} F_{n}$ consists of $m_{F}$ sets each congruent to $l_{F}^{n-1} F_{n-1}$. For $1 \leq i \leq l_{F}$ let $a_{i}$ be the number of sets congruent to $l_{F}^{n-1} F_{n-1}$ contained in $l_{F}^{n} F_{n} \cap\left\{x:(i-1) l_{F}^{n-1} \leq\right.$ $\left.x_{1} \leq i l_{F}^{n-1}\right\}$. Then $\sum a_{i}=m_{F}$, and applying shorts on each of the sets $\left\{x_{1}=i l_{F}^{n-1}\right\}$ we have from Kirchoff's laws

$$
R_{n} \geq R_{n-1} \sum_{i=1}^{l_{F}} a_{i}^{-1}
$$

Using (5.2) it follows that

$$
\rho_{F} \geq \sum_{i=1}^{l_{F}} a_{i}^{-1}
$$

By Cauchy-Schwarz,

$$
l_{F}^{2}=\left(\sum_{i=1}^{l_{F}} a_{i}^{\frac{1}{2}} a_{i}^{-\frac{1}{2}}\right)^{2} \leq\left(\sum_{i=1}^{l_{F}} a_{i}\right)\left(\sum_{i=1}^{l_{F}} a_{i}^{-1}\right)=m_{F}\left(\sum_{i=1}^{l_{F}} a_{i}^{-1}\right) .
$$

(a) is now clear.

(b) Using Hypothesis 2.1 (H4) we have that the two sides $\left\{x_{1}=0\right\}$ and $\left\{x_{1}=l_{F}^{n}\right\}$ of $l_{F}^{n} F_{n}$ are connected by $2^{d-1}$ disjoint blocks, each consisting of $l_{F}$ copies of $l_{F}^{n-1} F_{n-1}$ arranged in series. (In the case $d=3$ we are considering the cubes that touch the four edges connecting $\left\{x_{1}=0\right\}$ and $\left.\left\{x_{1}=l_{F}^{n}\right\}\right)$. Therefore

$$
R_{n} \leq R_{n-1} 2^{-(d-1)} l_{F},
$$

from which (b) follows immediately.

Remark 5.2 Note that while (a) is true for any GSC satisfying Hypotheses 2.1 (H1) and (H2), (b) relies on (H4), and does not always hold for more general GSCs.

Corollary 5.3 For GSCs which satisfy Hypotheses 2.1 (H1)-(H4) the following inequalities hold:

$$
\begin{gathered}
d_{w} \geq 2, \quad d_{s} \leq d_{f}<d, \\
d_{w} \leq 1+d_{f}-(d-1)\left(\log 2 / \log l_{F}\right)<1+d_{f} \\
d_{s}>\frac{2 d_{f}}{1+d_{f}}>1 .
\end{gathered}
$$


Proof (5.6) and (5.7) are immediate from Proposition 5.1 and the definitions of $d_{f}, d_{w}, d_{s}$. Hypothesis $2.1(\mathrm{H} 2)$ implies that $m_{F}>l_{F}$, so $d_{f}>1$, and (5.8) follows from (5.7).

Remarks 5.4 1. Note that the proof of Proposition 5.1(a) shows that $t_{F}>l_{F}^{2}$ provided that the $a_{i}$ are not all equal. In fact we have a proof that strict inequality holds in Proposition 5.1(a) for any GSC. However, the proof is rather longer than that given above. As a consequence, we know that $d_{w}>2$ and $d_{s}<d_{f}$ in (5.6).

2. Let $1 \leq b \leq a, d \geq 2$, and consider the GSC given by taking $l_{F}=a$, and $F_{1}=$ $F_{0}-((a-b) / 2 a,(a+b) / 2 a)^{d}$. So $F_{1}$ is obtained by removing a central block of $b^{d}$ cubes of side $a^{-1}$. We denote this GSC by SC $(a, b, d)$. We have $m_{F}=a^{d}-b^{d}$, and the estimates (5.4) and (5.5) give

$$
\frac{a-b}{a^{d-1}}+\frac{b}{a^{d-1}-b^{d-1}} \leq \rho_{F} \leq \frac{a}{a^{d-1}-b^{d-1}} .
$$

In the particular case of the standard 3-dimensional SC, $\operatorname{SC}(3,1,3)$, this implies that $25 / 72 \leq \rho_{F} \leq 27 / 72$, so that

$$
2.0028<d_{w}<2.0729, \quad 2.8614<d_{s}<2.9615 \text {. }
$$

This may be compared with the estimate

$$
d_{s} \geq d_{i}=\frac{\log 26}{\log (26 / 8)} \approx 2.7642
$$

obtained in [O1] using an isoperimetric inequality.

\section{Processes}

Let $\partial_{a} F_{n}=\left\{x \in F_{n}: x_{1} \vee \cdots \vee x_{d}=1\right\}$. We let $W_{t}^{n}$ be Brownian motion on $\tilde{F}_{n}$ with normal reflection on $\partial \tilde{F}_{n}$. Let $\tau_{n}^{W}=\inf \left\{t: W_{t}^{n} \in \partial_{a} F_{n}\right\}$. Let $G_{n}=\left\{x \in F_{n}: x_{1} \vee \cdots \vee x_{d} \leq \frac{1}{2}\right\}$. Then there exist $c_{1}, c_{2}$ such that for all $n \geq 0$

$$
c_{1}\left(t_{F} / l_{F}^{2}\right)^{n} \leq \inf _{x \in G_{n}} \mathbb{E}^{x} \tau_{n}^{W} \leq \sup _{x \in F_{n}} \mathbb{E}^{x} \tau_{n}^{W} \leq c_{2}\left(t_{F} / l_{F}^{2}\right)^{n}
$$

this is proved just as in [BB1, Prop. 4.2] and [BB2, Prop. 2.2 and 2.3]. We make the remark that $\left(t_{F} / l_{F}^{2}\right)^{-n}$ is also comparable to the first eigenvalue for the Laplacian on $F_{n}$ with Dirichlet boundary conditions on $\partial_{a} F_{n}$ and Neumann boundary conditions elsewhere; see [BB2, Prop. 2.2].

Let $X_{t}^{n}=W^{n}\left(\left(t_{F} / l_{F}^{2}\right)^{n} t\right)$ and let $\mathbb{P}_{n}^{x}$ be the law of $X_{t}^{n}$ when $X_{0}^{n}=x$. We have the following estimates on the $\mathbb{P}_{n} \cdot$ laws of $\sigma_{r}(x)=\sigma_{r}(x, X)$.

\section{Proposition 5.5}

(a) For $0 \leq r \leq n, x \in \tilde{F}_{n}$,

$$
c_{1} t_{F}^{-r} \leq \mathbb{E}_{n}^{x} \sigma_{r}(x) \leq c_{2} t_{F}^{-r}
$$


(b) Let $n \geq 0$. If $m \leq n$ and $t \geq l_{F}^{n-m} / t_{F}^{n}$, then

$$
\mathbb{P}_{n}^{x}\left(\sigma_{m}(x) \leq t\right) \leq c_{3} \exp \left(-c_{4}\left(t_{F}^{-m} / t\right)^{1 /\left(d_{w}-1\right)}\right) .
$$

$$
\text { If } m \leq n \text { and } t \geq l_{F}^{n-m} / t_{F}^{n} \text {, or if } m>n \text { and } t>0 \text {, then }
$$

$$
\mathbb{P}_{n}^{x}\left(\sigma_{m}(x) \leq t\right) \leq c_{5} \exp \left(-c_{6}\left(l_{F}^{2 n} / t_{F}^{n}\right)\left(l_{F}^{-2 m} / t\right)\right) .
$$

(c) For $x \in \tilde{F}_{n}, \lambda>l_{F}^{-n}$,

$$
\mathbb{P}_{n}^{x}\left(\sup _{s \leq t}\left|X_{s}^{n}-X_{0}^{n}\right| \geq \lambda\right) \leq c_{7} \exp \left(-c_{8}\left(\lambda^{d_{w}} / t\right)^{1 /\left(d_{w}-1\right)}\right) .
$$

Proof As the proofs are similar to those in [BB1, Prop. 4.4] and [BB4, (3.1), Prop. 3.3 and Theorem 3.4] we only give a sketch of the argument.

Note that (5.11) follows from (5.10) using scaling, which proves (a).

We turn to the proof of (b). It is easy to see (5.11) holds if $r \leq n+2$. By scaling we may suppose $n=0$ and $r>2$. In this case, $D_{r}(x)$ is a subset of the region above a Lipschitz function. Let $\bar{v}(y, z)$ be the Green function for a RBM in $\tilde{F}_{0}$ killed on exiting $D_{r}(x)$. By Corollary 3.3 of $[\mathrm{BH}]$ and integration,

$$
\mathbb{E}^{x} \sigma_{r}^{W}(x)=\int_{\tilde{F}_{0}} \bar{v}(x, y) d y \leq c_{9} l_{F}^{-2 r} .
$$

As in the proof of Proposition 2.5, if $d \geq 3$, there exists $K$ not depending on $r$ such that $\bar{v}(y, z) \geq c_{10}|y-z|^{2-d}$ for $y, z \in D_{r+K}(x)$. An integration then gives

$$
\mathbb{E}^{x} \sigma_{r}^{W}(x) \geq \int_{D_{r+K}(x)} \bar{v}(x, y) d y \geq c_{11} l_{F}^{-2 r} .
$$

The case $d=2$ is similar.

Since $X_{t}$ is a time change of $W_{t}$, we then have

$$
c_{12} t_{F}^{-n} l_{F}^{-2 r+2 n} \leq \mathbb{E}_{n}^{x} \sigma_{r}^{X}(x) \leq c_{13} t_{F}^{-n} l_{F}^{-2 r+2 n}, \quad r>n+2 .
$$

It follows as in [BB1, Lemma 4.3] that for $t \geq 0, x \in \tilde{F}_{n}$,

$$
\mathbb{P}_{n}^{x}\left(\sigma_{r}(x) \leq t\right) \leq c_{14}+c_{15} a_{n r} t
$$

where $c_{14} \in(0,1)$ and $a_{n r}=t_{F}^{n}$ if $r \leq n+2$, and $a_{n r}=l_{F}^{2 r-2 n} t_{F}^{n}$ if $r>n+2$. If $x(t)$ is a continuous path in $\tilde{F}_{n}$ we call an $r$-crossing a segment of the path $t_{1} \leq s<t_{2}$ where $x\left(t_{2}\right) \in \partial D_{n}\left(x\left(t_{1}\right)\right)$ and $x(s) \in D_{n}\left(x\left(t_{1}\right)\right)$ for $t_{1} \leq s<t_{2}$. If $m \leq r$ then any path from $x$ to $D_{m}(x)^{c}$ must include at least $l_{F}^{r-m} / 4$ disjoint $r$-crossings. So, writing $M=l_{F}^{r-m} / 4$, $\sigma_{m}(x) \geq \sum_{i=1}^{M} V_{i}$, where the $V_{i}$ satisfy the bounds in (5.15). By [BB1, Lemma 1.1],

$$
\log \mathbb{P}_{n}^{x}\left(\sigma_{m}(x) \leq t\right) \leq 2\left(a_{n r} M t / c_{14}\right)^{1 / 2}-M \log \left(1 / c_{14}\right) .
$$


It therefore follows that for $r \geq m$,

$$
\log \mathbb{P}^{x}\left(\sigma_{m}(x) \leq t\right) \leq c_{15}\left[\left(t_{F}^{r} l_{F}^{r-m} t\right)^{1 / 2}-l_{F}^{r-m}\right] \text { for } r \leq n+2
$$

and

$$
\log \mathbb{P}^{x}\left(\sigma_{m}(x) \leq t\right) \leq c_{16}\left[\left(l_{F}^{2 r-2 n} t_{F}^{n} l_{F}^{r-m} t\right)^{1 / 2}-l_{F}^{r-m}\right] \text { for } r>n+2
$$

The bounds in (b) then follow on choosing $r$ to minimize the right hand sides of (5.16) and (5.17).

(c) follows easily from (b); for a similar proof see, for example, [BB4, Theorem 3.4(b)].

Definition 5.6 Let $A$ and $B$ be Borel sets. For $A \subset \tilde{F}$ write $\partial_{\tilde{F}} A$ for the relative boundary of $A$ in $\tilde{F}$. A local isometry of $\tilde{F}$ is a triple $(\varphi, A, B)$ where $A, B \subset \tilde{F}$ and $\varphi$ is an isometry between $A$ and $B$ and also between $\partial_{\tilde{F}} A$ and $\partial_{\tilde{F}} B$. A Markov process $Y=\left((\mathbb{0})^{x}, Y_{t}\right), x \in$ $\tilde{F}, t>0$, is said to be invariant with respect to the local isometries of $\tilde{F}$ if for every local isometry $(\varphi, A, B)$ and $x \in A$,

$$
\mathbb{O}_{2}{ }^{x}\left(\varphi\left(Y_{t \wedge \tau(A)}\right) \in \cdot, t \geq 0\right)=\left(\mathbb{Q}^{\varphi(x)}\left(Y_{t \wedge \tau(B)} \in \cdot t \geq 0\right)\right.
$$

We will also say that $Y$ is locally isotropic.

As in [BB1] we have the theorem

Theorem 5.7 There exists a strong Markov process $X=\left(\mathbb{P}^{x}, X_{t}\right), x \in \tilde{F}, t \geq 0$, with state space $\tilde{F}$ such that $X$ has a Feller transition semigroup which is $\mu$-symmetric, $X$ has continuous paths, and the process $X$ is invariant with respect to the local isometries of $\tilde{F}$.

As a consequence of our results on the transition densities of $X_{t}$ later on, we shall see that $X_{t}$ actually has a strong Feller transition semigroup.

The proof of Theorem 5.7 is the same as that in [BB1, Sect. 6]. We briefly review the outline of the proof and refer the reader to that paper for details. Define

$$
U_{n}^{\lambda} f(x)=\mathbb{E}^{x} \int_{0}^{\infty} e^{-\lambda t} f\left(X_{t}^{n}\right) d t
$$

By the Harnack inequality and the modulus of continuity estimates for harmonic functions proved in Section 4 of this paper, it is not hard to see that when $f$ is bounded and continuous, then $\left\{U_{n}^{\lambda} f\right\}_{n=1}^{\infty}$ is equicontinuous on compact sets. By a diagonalization and limit argument, there exists a subsequence $n^{\prime}$ such that $U_{n^{\prime}}^{\lambda} f$ converges uniformly on compacts, say to $U^{\lambda} f$, for all $\lambda>0$ and $f$ bounded and continuous. Since $\left\{\mathrm{P}_{n^{\prime}}^{x}\right\}$ is tight, for each $x$ there exist convergent subsequences. Any limit point $\mathbb{P}^{x x}$ satisfies $\mathbb{E}^{x} \int_{0}^{\infty} e^{-\lambda t} f\left(X_{t}\right) d t=U^{\lambda} f(x)$ for $f$ bounded and continuous, from which one deduces that $\mathrm{PP}_{n^{\prime}}^{x}$ converges. If one calls the limit $\mathbb{P}^{x}$ and lets $X_{t}$ be the canonical process on $F$, one then can show that $\left(\mathbb{P}^{x}, X_{t}\right)$ has the strong Markov property as well as the other required properties. It is then straightforward to extend $\left(\mathbb{P}^{x}, X_{t}\right)$ to a process on $\tilde{F}$. 
Many properties of $X$ follow almost immediately from the corresponding properties of the $X^{n}$. Thus from Proposition 5.5 we deduce

Proposition 5.8 For $r \in \mathbb{Z}, t>0$,

$$
\begin{aligned}
\mathbb{P}^{x}\left(\sigma_{r}(x) \leq t\right) & \leq c_{1} \exp \left(-c_{2}\left(t_{F}^{r} t\right)^{-1 /\left(d_{w}-1\right)}\right) \\
c_{3} t_{F}^{-r} & \leq \mathbb{E}^{x} \sigma_{r}(x) \leq c_{4} t_{F}^{-r}
\end{aligned}
$$

and

$$
\mathbb{P}^{x}\left(\sup _{s \leq t}\left|X_{s}-X_{0}\right| \geq \lambda\right) \leq c_{5} \exp \left(-c_{6}\left(\lambda^{d_{w}} / t\right)^{1 /\left(d_{w}-1\right)}\right), \quad \lambda>0 .
$$

We also have the weak scaling property: the $1 l^{r} l_{F}^{x}$ law of $l_{F}^{-r} X\left(t_{F}^{r} t\right)$ satisfies the estimates (5.19)-(5.21).

Let $P_{t}$ be the semigroup of the process $X_{t}$ (acting on $\left.L^{2}(\tilde{F}, \mu)\right)$, and $(\mathcal{L}, \mathcal{D}(\mathcal{L}))$ be the infinitesimal generator of $\left(P_{t}\right)$. Since $X$ is $\mu$-symmetric, we have for $f, g \in L^{2}(\tilde{F}, \mu)$,

$$
\int f(x) P_{t} g(x) \mu(d x)=\int P_{t} f(x) g(x) \mu(d x) .
$$

By [FOT] there exists a Dirichlet form $\mathcal{E}$ with domain $\mathcal{D}(\mathcal{E}) \subset L^{2}(\tilde{F}, \mu)$ associated with the semigroup $\left(P_{t}\right)$. See [FOT, Chapter 1] for details of the relations between $X, P_{t}$ and $(\mathcal{E}, \mathcal{D}(\mathcal{E}))$; here we just note that $\mathcal{D}(\mathcal{L})$ is dense in $\mathcal{D}(\mathcal{E})$ and that

$$
\mathcal{E}(f, g)=-\int \mathcal{L} f(x) g(x) \mu(d x), \quad f \in \mathcal{D}(\mathcal{L}), \quad g \in \mathcal{D}(\mathcal{E}) .
$$

If $h: \tilde{F} \rightarrow \mathbb{R}$ we say $h$ is harmonic (with respect to $X$ ) if $h \in \mathcal{D}(\mathcal{L})$ and $\mathcal{L} h=0$, or, equivalently, if $h\left(X_{t}\right)$ is a local martingale.

Theorem 5.9 Let $x_{0} \in \tilde{F}, n \in \mathbb{Z}$, and $h$ be nonnegative and harmonic in $D_{n}\left(x_{0}\right) \cap \tilde{F}$. There exist $c_{1}, c_{2}$ and $\alpha$, depending only on $d$ and $l_{F}$ such that

(a)

$$
h(x) \leq c_{2} h(y), \quad x, y \in D_{n+4}\left(x_{0}\right)
$$

$$
|h(x)-h(y)| \leq c_{1}|x-y|^{\alpha} l_{F}^{n \alpha} \sup _{D_{n}\left(x_{0}\right)}|h|, \quad x, y \in D_{n+2}\left(x_{0}\right),
$$

Proof (a) By (5.19) we have that $X_{t}$ leaves a point $x$ immediately. It follows from the symmetry of $X$ that if $x \in \partial D_{m}(0)$, then $\mathbb{P}^{x}\left(\tau_{D_{m}(0)}>0\right)>0$, and by the Blumenthal 0-1 law this probability must be 1 . This shows that if $f$ is a continuous function on $\partial D_{m}(0)$, then $f\left(X_{\sigma_{m}(0)}\right)$ is a continuous functional of the path except for a null set, so $u_{n}(x)=\mathbb{E}_{n}^{x} f\left(X_{\sigma_{m}(0)}\right)$ converges (along a subsequence) to $u(x)=\mathbb{E}^{x} f\left(X_{\sigma_{m}(0)}\right)$. By Theorem 4.3 the functions $u_{n}$ 
satisfy (5.23) with constants independent of $n$, and therefore $u$ also satisfies (5.23). This proves the result when $u$ is non-negative and continuous on $D_{n}\left(x_{0}\right) \cap \tilde{F}$; by a limit argument we then obtain the result for all positive harmonic functions.

(b) This is standard using an argument of Moser [M].

Remark 5.10 By very analogous methods, we can construct a process $\hat{X}$ on domains of the form $\tilde{F} \cap D_{n}\left(x_{0}\right)$ with normal reflection on $\partial D_{n}\left(x_{0}\right)$. We let $\widehat{W}_{t}^{m}$ be $\operatorname{RBM}\left(\tilde{F}_{0} \cap D_{m}\left(x_{0}\right)\right)$, set $\hat{X}_{t}=\widehat{W}^{m}\left(\left(t_{F} / l_{F}^{2}\right)^{n} t\right)$, and proceed as in the proof of Theorem 5.7.

For use in Section 6 we note the estimates,

$$
\mathbb{P}^{x}\left(\sigma_{r}(x, \hat{X}) \leq t\right) \leq c_{1} \exp \left(-c_{2}\left(t_{F}^{r} t\right)^{-1 /\left(d_{w}-1\right)}\right), \quad t>0, r \geq n,
$$

and

$$
\mathbb{E}^{x} \sigma_{r}(x, \hat{X}) \leq c_{3} t_{F}^{-r}, \quad r \geq n
$$

Remark 5.11 Note that the process $X$ in Theorem 5.7 is only obtained as a subsequential limit of the processes $X^{n}$. If we had $X=\lim X^{n}$ then it would follow easily that $X$ was self-similar, that is, that the $\mathbb{P}^{x x}$ law of $l_{F}^{-1} X_{t}$ would be equal to the $\mathbb{P}^{p x} / l_{F}$ law of $X_{t / t_{F}}$. See Section 9 for some further remarks on this point.

However, a self-similar process $Y_{t}$ on $\tilde{F}$ can be constructed as follows. By Proposition 5.1 we have $\rho_{F} m_{F} \geq l_{F}^{2}$, so that $\rho_{F} \geq l_{F}^{d} m_{F}^{-d} l_{F}^{2-d} \geq l_{F}^{1-d}$. So $F$ satisfies the "good borders" hypothesis in [KZ], and we may now follow the argument of [KZ] to construct a diffusion $Y_{t}$ on $\tilde{F}$ that is also self-similar. We refer the reader to [KZ] for details. Briefly, in that paper $\mathcal{E}_{n}$ is defined to be the Dirichlet form for a graphical approximation to $Y^{n}, \frac{1}{n} \sum_{j=1}^{n} \mathcal{E}_{j}$ is shown to have subsequential limit points, and if $\mathcal{E}$ is one of those subsequential limit points, it is shown that $\mathcal{E}$ is a closable Dirichlet form. The process $Y_{t}$ is then the Markov process associated with $\mathcal{E}$.

\section{Transition Density Estimates}

Let $\left(\mathbb{P}^{x}, W_{t}\right)$ be a $\operatorname{RBM}\left(\tilde{F}_{0}\right)$. We have the following estimates on the transition densities of $W_{t}$.

Proposition 6.1 $W_{t}$ has continuous transition densities $q(t, x, y)$ with respect to Lebesgue measure $\mu_{0}$ on $\tilde{F}_{0}$, which satisfy

$$
q(t, x, y)=q(t, y, x) \leq q(t, x, x)^{\frac{1}{2}} q(t, y, y)^{\frac{1}{2}},
$$

$q(t, x, x)$ is nonincreasing in $t$ for each $x \in \tilde{F}_{0}$, and for each $t_{0}$

$$
q(t, x, y) \leq c_{1}\left(t_{0}\right) t^{-d / 2}, \quad 0 \leq t \leq t_{0}, \quad x, y \in \tilde{F}_{0} .
$$

Proof Fix $x, y \in \tilde{F}_{0}$ and select $m<0$ so that $x, y \in D_{m+4}\left(x_{0}\right) \cap \tilde{F}_{0}$. Let $C$ be a small subset of $D_{m+4}\left(x_{0}\right) \cap \tilde{F}_{0}$ containing $y$. Let $S_{1}=\sigma_{m}\left(x_{0}\right), U_{i}=\inf \left\{t>S_{i}: W_{t} \in D_{m+2}\left(x_{0}\right)\right\}$, and 
$S_{i+1}=\inf \left\{t>U_{i}: W_{t} \notin D_{m}\left(x_{0}\right)\right\}$. Then

$$
\begin{aligned}
\mathbb{P}^{x}\left(W_{t} \in C\right)= & \mathbb{P}^{x}\left(W_{t} \in C, t<S_{1}\right)+\mathbb{P}^{x}\left(W_{t} \in C, U_{1}<t<S_{2}\right) \\
& +\mathbb{P}^{x}\left(W_{t} \in C, U_{2}<t<S_{3}\right)+\cdots
\end{aligned}
$$

If $\bar{q}(t, x, y)$ is the transition density for $W_{t}$ killed on exiting $D_{m}\left(x_{0}\right)$, the first term on the right of (6.3) is

$$
\int_{C} \bar{q}(t, x, z) d z
$$

The second term is

$$
\int_{C} \mathbb{E}^{x}\left[\int_{0}^{t} \bar{q}\left(t-s, W_{U_{1}}, z\right) ; U_{1} \in d s\right] d z
$$

Note that this is less than

$$
\sup _{w \in \partial D_{m+2}\left(x_{0}\right)} \sup _{z \in D_{m+4}\left(x_{0}\right)} \sup _{r \leq t} \bar{q}(r, w, z) \mathbb{P}^{x}\left(U_{1} \leq t\right) \mu_{0}(C),
$$

and the succeeding terms of (6.3) are bounded similarly. Provided $t$ is small enough, (5.13) and (2.10) imply that the $i$-th term of (6.3) is bounded by $c_{2} \rho^{i} t^{-d / 2} \mu_{0}(C)$, where $\rho<1$. Therefore $\mathbb{P}^{x}\left(W_{t} \in d y\right)$ is absolutely continuous with respect to Lebesgue measure, the density $q(t, x, y)$ satisfies

$$
q(t, x, z)=\bar{q}(t, x, z)+\mathbb{E}^{x}\left[\int_{0}^{t} \bar{q}\left(t-s, W_{U_{1}}, z\right) ; U_{1} \in d s\right]+\cdots
$$

and the series is uniformly convergent in a neighborhood of $y$. Since for each $w$ we know that $\bar{q}(t, w, z)$ is continuous in $z$, this implies that $q(t, x, z)$ is continuous in $z$ for $z$ in a neighborhood of $y$.

Taking $y=x$, we obtain from (6.5) that

$$
q(t, x, x) \leq c_{3} t^{-d / 2}, \quad t \leq t_{1}, \quad x \in \tilde{F}_{0} .
$$

Since $q(t, x, y)<\infty$ for $t$ small enough, the semigroup property shows that $q(t, x, y)<\infty$ for all $t$. Letting $m \rightarrow-\infty$, (2.13) implies (6.1). Finally, (6.2) is implied by (6.6), (6.1), and the semigroup property.

Proposition 6.2 There exist $c_{1}$ and $c_{2}$ such that $q(t, x, y) \geq c_{1} t^{-d / 2}$ if $|x-y| \leq c_{2} t^{\frac{1}{2}}, t \leq 1$.

Proof Let $\bar{q}(t, x, y)$ be the transition density for $W$ killed on exiting $\tilde{F}_{0} \cap D_{4}\left(x_{0}\right)$. Let $D^{\prime}$ be the region above the graph of a Lipschitz function in some coordinate system such that $D^{\prime} \cap D_{4}\left(x_{0}\right)=\tilde{F}_{0} \cap D_{4}\left(x_{0}\right)$. Let $q^{\prime}(t, x, y)$ be the transition densities for RBM in $D^{\prime}$. Let $S_{i}$ and $U_{i}$ be defined as in Proposition 6.1, and as in (6.5),

$$
q^{\prime}(t, x, y)=\bar{q}(t, x, y)+\mathbb{E}^{x}\left[\int_{0}^{t} q^{\prime}\left(t-s, W_{U_{1}}, y\right) ; U_{1} \in d s\right], \quad x, y \in D_{8}\left(x_{0}\right)
$$


By $[\mathrm{BH}]$, Theorem 3.4,

$$
q^{\prime}(t, x, y) \geq c_{3} t^{-d / 2} \exp \left(-c_{4}|x-y|^{2} / t\right), \quad x, y \in D_{8}\left(x_{0}\right)
$$

while $[\mathrm{BH}]$, Theorem 3.1 says that

$$
q^{\prime}(t-s, w, y) \leq c_{5} t^{-d / 2} \exp \left(-c_{6}|w-y|^{2} /(t-s)\right)
$$

Since $\mathbb{P}^{x}\left(U_{1} \leq t\right) \leq c_{7} \exp \left(-c_{8} / t\right)$ by (5.13) for $t$ sufficiently small and $x \in D_{8}\left(x_{0}\right)$, then if we take $t_{0}$ sufficiently small

$$
\bar{q}(t, x, y) \geq c_{9} t^{-d / 2} \exp \left(-c_{10}|x-y|^{2} / t\right), \quad x, y \in D_{8}\left(x_{0}\right), \quad t \leq t_{0},
$$

and so

$$
\bar{q}(t, x, y) \geq c_{11} t^{-d / 2}, \quad x, y \in D_{8}\left(x_{0}\right), \quad|x-y| \leq c_{12} t^{\frac{1}{2}}, \quad t \leq t_{0} .
$$

The proposition now follows by repeated applications of the semigroup property.

Lemma 6.3 Let $x \in \tilde{F}_{0}$. Then writing $\sigma_{-n}(x)=\sigma_{-n}(x, W)$,

$$
\begin{gathered}
\mathbb{P}^{x}\left(\sigma_{-n}(x) \leq t\right) \leq c_{1} \exp \left(-c_{2}\left(t t_{F}^{-n}\right)^{-1 /\left(d_{w}-1\right)}\right), \quad t \geq l_{F}^{n}, \quad n \geq 0, \\
\mathbb{P}^{x}\left(\sigma_{-n}(x) \leq t\right) \leq c_{3} \exp \left(-c_{4} l_{F}^{2 n} / t\right), \quad 0 \leq t \leq l_{F}^{n}, \quad n \in \mathbb{Z}, \\
\mathbb{P}^{y}\left(\sigma_{-n}(x) \geq t\right) \leq c_{5} \exp \left(-c_{6} t t_{F}^{-n}\right), \quad t>0, \quad x \in \tilde{F}_{0}, \quad y \in D_{-n}(x), \quad n \geq 0 .
\end{gathered}
$$

Proof (6.7) and (6.8) are just restatements of (5.12) and (5.13). To prove (6.9), note first the estimate $\mathbb{E}^{y} \sigma_{-n}(y) \leq c_{7} t_{F}^{n}$ given in (5.11). Let $t_{0}=2 c_{7}^{-1} t_{F}^{2}$. Then $\mathbb{P}^{y}\left(\sigma_{-n-2}(y)>\right.$ $\left.t_{0} t_{F}^{n}\right) \leq \frac{1}{2}$ for $y \in \tilde{F}_{0}$. If $y \in D_{-n}(x)$, then $\sigma_{-n-2}(y) \geq \sigma_{-n}(x)$, so $\mathbb{P}^{y}\left(\sigma_{-n}(x)>t_{0} t_{F}^{n}\right) \leq \frac{1}{2}$. Iterating and using the strong Markov property we deduce that $\mathbb{P}^{y}\left(\sigma_{-n}(x)>k t_{0} t_{F}^{n}\right) \leq 2^{-k}$ for $k \geq 1$, and (6.9) follows easily.

Integrating these bounds we obtain:

\section{Lemma 6.4}

(a) There exists $c_{1}=c_{1}(p)$ such that

$$
\mathbb{E}^{y} \sigma_{-n}(x)^{p} \leq c_{1} t_{F}^{p n}, \quad y \in D_{-n}(x), \quad p>0, \quad n \geq 0 .
$$

(b) There exist constants $c_{2}, c_{3}$, such that

$$
\mathbb{E}^{x} e^{-\lambda \sigma_{-n}(x)} \leq c_{2} \exp \left(-c_{3}\left(\lambda t_{F}^{n}\right)^{1 / d_{w}}\right), \quad 0 \leq \lambda \leq 1, \quad n \geq 0
$$

Lemma 6.5 There exists $c_{1}$ such that if $x_{0} \in \tilde{F}_{0}$ and $A \subset D_{6}\left(x_{0}\right)$ then

$$
\begin{gathered}
\mathbb{E}^{y} \int_{0}^{\sigma-n\left(x_{0}\right)} 1_{A}\left(W_{s}\right) d s \leq c_{1} \mu_{0}(A) t_{F}^{n\left(1-d_{s} / 2\right)}, \quad n \geq 0 \\
y \in\left(D_{-n}\left(x_{0}\right)-D_{-n+2}\left(x_{0}\right)\right) \cap \tilde{F}_{0}
\end{gathered}
$$


Proof Let $n, x_{0}$ be fixed, let $\bar{q}_{n}(t, x, y)$ be the transition density of $W$ killed on exiting $D_{-n}\left(x_{0}\right)$, and let $\bar{v}(x, y)=\int \bar{q}_{n}(t, x, y) d t$. So

$$
\mathbb{E}^{y} \int_{0}^{\sigma_{-n}\left(x_{0}\right)} 1_{A}\left(W_{s}\right) d s=\int_{A} \bar{v}(y, z) \mu_{0}(d z) .
$$

Let $z \in A$. Then $\bar{v}(\cdot, z)$ is harmonic on $\left(D_{-n}\left(x_{0}\right)-\{z\}\right) \cap \tilde{F}_{0}$, and is zero on the boundary of $D_{-n}\left(x_{0}\right)$. Thus it is sufficient to bound (6.12) in the case $y \in \partial D_{-n+2}\left(x_{0}\right)$. So let $y_{0} \in$ $\partial D_{-n+2}\left(x_{0}\right) \cap \tilde{F}_{0}$. Note that if $m=-n+3$ then $D_{m}\left(y_{0}\right) \subset D_{-n}\left(x_{0}\right)$, and $D_{m}\left(y_{0}\right) \cap$ $D_{-n+4}\left(x_{0}\right)=\varnothing$. So $\bar{v}(\cdot, z)$ is harmonic on $D_{m}\left(y_{0}\right)$ and applying Theorem 4.3 in $D_{m}\left(y_{0}\right)$ we have

$$
\bar{v}\left(y_{0}, z\right) \leq c_{2} \bar{v}(y, z), \quad y \in D_{m+2}\left(y_{0}\right) .
$$

Therefore

$$
\begin{aligned}
\bar{v}\left(y_{0}, z\right) & \leq c_{2} \mu_{0}\left(D_{m+2}\left(y_{0}\right)\right)^{-1} \int_{D_{m+2}\left(y_{0}\right)} \bar{v}(y, z) \mu_{0}(d y) \\
& \leq c_{3} m_{F}^{-n} \int_{D_{-n}\left(x_{0}\right)} \bar{v}(y, z) \mu_{0}(d y) \\
& =c_{3} t_{F}^{-n d_{s} / 2} \mathbb{E}^{z} \sigma_{-n}\left(x_{0}\right) \leq c_{4} t_{F}^{-n d_{s} / 2} t_{F}^{n} .
\end{aligned}
$$

As this estimate holds for any $z \in A$, using (6.12) proves the lemma.

Lemma 6.6 Let $p>0$. There exists $c_{1}=c_{1}(p)$ such that if $x_{0} \in \tilde{F}_{0}$ and $A \subset D_{6}\left(x_{0}\right)$ then

$$
\mathbb{E}^{y} \int_{0}^{\sigma_{-n}\left(x_{0}\right)} t^{p} 1_{A}\left(W_{t}\right) d t \leq c_{1} \mu_{0}(A) t_{F}^{n\left(1+p-d_{s} / 2\right)}, \quad n \geq 0, \quad y \in \partial D_{-n+1}\left(x_{0}\right) .
$$

Proof Fix $x_{0}$ and $A$, and let $\bar{q}_{n}(t, x, y)$ be the transition density of $W$ killed on exiting $D_{-n}\left(x_{0}\right)$. Set

$$
w(x, y)=\int_{0}^{\infty} \bar{q}_{n}(t, x, y) t^{p} d t
$$

note that $w(x, y)=w(y, x)$. Set

$$
g(y)=\mathbb{E}^{y} \int_{0}^{\sigma_{-n}\left(x_{0}\right)} t^{p} 1_{A}\left(W_{t}\right) d t=\int_{A} w(y, z) \mu_{0}(d z) .
$$

Let $y_{0} \in \partial D_{-n+2}\left(x_{0}\right)$. Then $D_{-n+4}\left(y_{0}\right) \cap A=\varnothing$, and so if $y \in D_{-n+4}\left(y_{0}\right)$, then writing $T=\sigma_{-n+4}\left(y_{0}\right)$, and using Lemmas 6.4 and 6.5,

$$
\begin{aligned}
g(y) & =\mathbb{E}^{y} \int_{T}^{\sigma_{-n}\left(x_{0}\right)} t^{p} 1_{A}\left(W_{t}\right) d t \\
& \leq c_{2} \mathbb{E}^{y}\left(T^{p} \mathbb{E}^{W_{T}} \int_{0}^{\sigma_{-n}\left(x_{0}\right)} 1_{A}\left(W_{t}\right) d t\right)+c_{3} \mathbb{E}^{y}\left(\mathbb{E}^{W_{T}} \int_{0}^{\sigma_{-n}\left(x_{0}\right)} t^{p} 1_{A}\left(W_{t}\right) d t\right) \\
& \leq c_{4} \mu_{0}(A)\left(t_{F}^{n}\right)^{p+1-d_{s} / 2}+c_{3} \mathbb{E}^{y} g\left(W_{T}\right) .
\end{aligned}
$$


Now let

$$
h(y)=\mathbb{E}^{y} g\left(W_{T}\right) .
$$

$h$ is harmonic on $D_{-n+4}\left(y_{0}\right)$, so applying Theorem 4.3 in $D_{-n+4}\left(y_{0}\right)$, we have

$$
h\left(y_{0}\right) \leq c_{5} h(y), \quad y \in D_{-n+6}\left(y_{0}\right) .
$$

Using the definitions of $g$ and $h$ and the strong Markov property,

$$
\begin{aligned}
h(y) & =\mathbb{E}^{y} g\left(W_{T}\right) \\
& =\mathbb{E}^{y} \mathbb{E}^{W_{T}} \int_{0}^{\sigma_{-n}\left(x_{0}\right)} t^{p} 1_{A}\left(W_{T}\right) d t \\
& =\mathbb{E}^{y} \int_{T}^{\sigma_{-n}\left(x_{0}\right)}(t-T)^{p} 1_{A}\left(W_{t}\right) d t \\
& \leq \mathbb{E}^{y} \int_{0}^{\sigma_{-n}\left(x_{0}\right)} t^{p} 1_{A}\left(W_{T}\right) d t=g(y) .
\end{aligned}
$$

We deduce that

$$
\begin{aligned}
h\left(y_{0}\right) & \leq c_{5} \mu_{0}\left(D_{-n+6}\left(y_{0}\right)\right)^{-1} \int_{D_{-n+6}\left(y_{0}\right)} g(y) \mu_{0}(d y) \\
& \leq c_{6} m_{F}^{-n} \int_{D_{-n}\left(y_{0}\right)} \int_{A} w(y, z) \mu_{0}(d z) \mu_{0}(d y) \\
& =c_{6} m_{F}^{-n} \int_{A} \mu_{0}(d z) \int_{D_{-n}\left(y_{0}\right)} w(z, y) \mu_{0}(d y) .
\end{aligned}
$$

However,

$$
\int_{D_{-n}\left(y_{0}\right)} w(z, y) \mu_{0}(d y)=\mathbb{E}^{z} \int_{0}^{\sigma_{-n}\left(x_{0}\right)} t^{p} d t=c_{7} \mathbb{E}^{z} \sigma_{-n}\left(x_{0}\right)^{p+1} \leq c_{8}\left(t_{F}^{n}\right)^{1+p}
$$

Therefore

$$
h\left(y_{0}\right) \leq c_{9} \mu_{0}(A)\left(t_{F}^{n}\right)^{1+p-d_{s} / 2},
$$

and using (6.13) it follows that

$$
\mathbb{E}^{y_{0}} \int_{0}^{\sigma_{-n}\left(x_{0}\right)} t^{p} 1_{A}\left(W_{t}\right) d t \leq c_{10} \mu_{0}(A)\left(t_{F}^{n}\right)^{1+p-d_{s} / 2}
$$

for $y_{0} \in \partial D_{-n+2}\left(x_{0}\right) \cap \tilde{F}_{0}$. Now if $y_{1} \in \partial D_{-n+1}\left(x_{0}\right)$, then applying (6.14) in the case $n+1$ we have

$$
\begin{aligned}
\mathbb{E}^{y_{1}} \int_{0}^{\sigma_{-n}\left(x_{0}\right)} t^{p} 1_{A}\left(W_{t}\right) d t & \leq \mathbb{E}^{y_{1}} \int_{0}^{\sigma_{-n-1}\left(x_{0}\right)} t^{p} 1_{A}\left(W_{t}\right) d t \\
& \leq c_{11} \mu_{0}(A)\left(t_{F}^{n}\right)^{1+p-d_{s} / 2}
\end{aligned}
$$


proving the lemma.

Choose $p>d>d_{s}$, and for $\lambda>0$ write

$$
g_{\lambda, p}(x, y)=\int_{0}^{\infty} q(t, x, y) t^{p} e^{-\lambda t} d t .
$$

Proposition 6.7 There exists $c_{1}=c_{1}(p)$ such that

$$
g_{\lambda, p}(x, x) \leq c_{1} \lambda^{-p-1+d_{s} / 2}, \quad x \in \tilde{F}_{0}, \lambda \leq 1 .
$$

Proof (In this proof, we suppress the dependence of the constants $c_{i}$ on $p$.) Fix $x \in \tilde{F}_{0}$, and write $\sigma_{-n}=\sigma_{-n}(x)$. Let $A \subset D_{6}(x)$, and set

$$
\begin{aligned}
V(A) & =\mathbb{E}^{x} \int_{0}^{\infty} 1_{A}\left(W_{s}\right) s^{p} e^{-\lambda s} d s \\
& =\mathbb{E}^{x} \int_{0}^{\sigma_{0}} 1_{A}\left(W_{t}\right) t^{p} e^{-\lambda t} d t+\sum_{n=0}^{\infty} \mathbb{E}^{x} \int_{\sigma_{-n}}^{\sigma_{-n-1}} 1_{A}\left(W_{t}\right) t^{p} e^{-\lambda t} d t .
\end{aligned}
$$

Write $\bar{q}(t, x, y)$ for the transition density of $W$ killed at $\sigma_{0}(x)$. Then by (2.12)

$$
\bar{q}(t, x, y) \leq c_{2} t^{-d / 2} e^{-c_{3} t}, \quad t>0 .
$$

Therefore

$$
\begin{aligned}
\mathbb{E}^{x} \int_{0}^{\sigma_{0}(x)} 1_{A}\left(W_{t}\right) t^{p} e^{-\lambda t} d t & =\int_{A} \int_{0}^{\infty} \bar{q}(t, x, y) t^{p} e^{-\lambda t} d t \mu_{0}(d y) \\
& \leq c_{2} \int_{A} \mu_{0}(d y) \int_{0}^{\infty} t^{p-d / 2} e^{-c_{3} t} d t=c_{4} \mu_{0}(A) .
\end{aligned}
$$

Write $J_{n}$ for the $n$-th term of the sum in (6.15), and note that

$$
\begin{aligned}
J_{n}= & \mathbb{E}^{x}\left(e^{-\lambda \sigma_{-n}} \mathbb{E}^{W_{\sigma_{-n}}} \int_{0}^{\sigma_{-n-1}} 1_{A}\left(W_{t}\right)\left(\sigma_{-n}+t\right)^{p} e^{-\lambda t} d t\right) \\
\leq & c_{5}\left(\mathbb{E}^{x} e^{-\lambda \sigma_{-n}} \sigma_{-n}^{p} \mathbb{E}^{W_{\sigma_{-n}}} \int_{0}^{\sigma_{-n-1}} 1_{A}\left(W_{t}\right) d t\right) \\
& +c_{5} \mathbb{E}^{x}\left(e^{-\lambda \sigma_{-n}} \mathbb{E}^{W_{\sigma_{-n}}} \int_{0}^{\sigma_{-n-1}} t^{p} 1_{A}\left(W_{t}\right) d t\right) .
\end{aligned}
$$

Using the bounds in Lemmas 6.4-6.6, we therefore have

$$
\begin{aligned}
J_{n} \mu_{0}(A)^{-1} & \leq c_{5} \mathbb{E}^{x}\left(\sigma_{-n}^{p} e^{-\lambda \sigma_{-n}}\right) t_{F}^{n\left(1-d_{s} / 2\right)}+c_{5} \mathbb{E}^{x}\left(e^{-\lambda \sigma_{-n}}\right) t_{F}^{n\left(1+p-d_{s} / 2\right)} \\
& \leq c_{5} t_{F}^{n\left(1-d_{s} / 2\right)}\left(\left(\mathbb{E}^{x}\left(\sigma_{-n}^{2 p}\right)\right)^{1 / 2}\left(E^{x}\left(e^{-\lambda \sigma_{-n}}\right)\right)^{1 / 2}+t_{F}^{n p} E^{x} e^{-\lambda \sigma_{-n}}\right) \\
& \leq c_{6} t_{F}^{n\left(1+p-d_{s} / 2\right)} \exp \left(-c_{7}\left(\lambda t_{F}^{n}\right)^{1 / d_{w}}\right) .
\end{aligned}
$$


Writing $\beta=1+p-d_{s} / 2$, we deduce from the calculations above that

$$
V(A) \leq c_{8} \mu_{0}(A)\left(1+\sum_{n=0}^{\infty}\left(t_{F}^{\beta}\right)^{n} \exp \left(-c_{9}\left(\lambda t_{F}^{n}\right)^{1 / d_{w}}\right)\right)
$$

Let $m_{0}$ be such that $t_{F}^{m_{0}} \geq 1 / \lambda>t_{F}^{m_{0}-1}$. Then, as $\beta>0$, the sum above is bounded by $c_{10} \mu_{0}(A) t_{F}^{m_{0} \beta}$. So

$$
V(A)=\int_{A} g_{\lambda, p}(x, y) \mu_{0}(d y) \leq c_{11} \mu_{0}(A) \lambda^{-1-p+d_{s} / 2}
$$

and as $g_{\lambda, p}(x, y)$ is continuous, this proves the proposition.

Theorem 6.8 For $x \in \tilde{F}_{0}$,

$$
q(t, x, y) \leq \begin{cases}c_{1} t^{-d / 2}, & 0<t \leq 1, \\ c_{1} t^{-d_{s} / 2}, & 1 \leq t<\infty .\end{cases}
$$

Proof The bound for $t \in(0,1]$ is given by (6.2). If $t \geq 1$, let $\lambda=t^{-1}$. Since $q(t, x, x)$ is non-increasing in $t$,

$$
\begin{aligned}
c_{2} t^{1+p-d_{s} / 2} & \geq g_{1 / t, p}(x, x) \\
& \geq \int_{t / 2}^{t} q(s, x, x) s^{p} e^{-s / t} d s \\
& \geq q(t, x, x) \int_{t / 2}^{t} s^{p} e^{-s / t} d s=c_{3} t^{1+p} q(t, x, x) .
\end{aligned}
$$

This proves the theorem if $x=y$, and the general case now follows from (6.1).

Theorem 6.9 There exist $c_{1}, \ldots, c_{8} \in(0, \infty)$ such that if $x, y \in \tilde{F}_{0}$ and

(a) $t \geq 1,|x-y| \leq t$, then

$$
\begin{aligned}
c_{1} t^{-d_{s} / 2} \exp \left(-c_{2}\left(\frac{|x-y|^{d_{w}}}{t}\right)^{1 /\left(d_{w}-1\right)}\right) \\
\leq q(t, x, y) \leq c_{3} t^{-d_{s} / 2} \exp \left(-c_{4}\left(\frac{|x-y|^{d_{w}}}{t}\right)^{1 /\left(d_{w}-1\right)}\right)
\end{aligned}
$$

(b) if $t \geq 1,|x-y| \geq t$, or if $t \leq 1$, then writing $D(t)=d, t \leq 1, D(t)=d_{s}$ for $t>1$,

$$
c_{5} t^{-D(t) / 2} \exp \left(-c_{6}|x-y|^{2} / t\right) \leq q(t, x, y) \leq c_{7} t^{-D(t) / 2} \exp \left(-c_{8}|x-y|^{2} / t\right) .
$$


Proof The argument for the upper bounds in (a) and (b) follows that of [BB4] quite closely.

Fix $x, y, t$, and choose small neighborhoods $C_{x}, C_{y}$ of $x$ and $y$. Let $A_{x}=\left\{z \in \tilde{F}_{0}\right.$ : $|x-z| \leq|x-y|\}$, and define $A_{y}$ similarly. Let $\nu_{x}=\left.\mu_{0}\right|_{C_{x}}, \nu_{y}=\left.\mu_{0}\right|_{C_{y}}$, and let

$$
S=\inf \left\{t \geq 0:\left|W_{t}-W_{0}\right|>\frac{1}{3}|x-y|\right\} .
$$

As $\mu_{0}\left(A_{x} \cap A_{y}\right)=0$,

$$
\begin{aligned}
\int_{C_{y}} \int_{C_{x}} q\left(t, x^{\prime}, y^{\prime}\right) \mu_{0}\left(d x^{\prime}\right) \mu_{0}\left(d y^{\prime}\right)= & \mathbb{P}^{\nu_{x}}\left(W_{t} \in C_{y}\right) \\
= & \mathbb{P}^{\nu_{x}}\left(W_{t} \in C_{y}, W_{t / 2} \in A_{x}\right) \\
& \quad+\mathbb{P}^{\nu_{x}}\left(W_{t} \in C_{y}, W_{t / 2} \in A_{y}\right) .
\end{aligned}
$$

We begin with the second term in (6.18).

$$
\begin{aligned}
\mathbb{P}^{\nu_{x}}\left(W_{t} \in C_{y}, W_{t / 2} \in A_{y}\right) & =\mathbb{P}^{\nu_{x}}\left(S<t / 2, W_{t} \in C_{y}, W_{t / 2} \in A_{y}\right) \\
& \leq \mathbb{P}^{\nu_{x}}(S<t / 2) \sup _{y^{\prime} \in A_{y}} \mathbb{P}^{y^{\prime}}\left(W_{t / 2} \in C_{y}\right) \\
& \leq \mu_{0}\left(C_{x}\right) \sup _{x^{\prime} \in C_{x}} \mathbb{P}^{x^{\prime}}(S<t / 2) \sup _{y^{\prime} \in A_{y}} \mathbb{P}^{y^{\prime}}\left(W_{t / 2} \in C_{y}\right) .
\end{aligned}
$$

For the first term in (6.18) we have, using the symmetry of $W$,

$$
\mathbb{P}^{\nu_{x}}\left(W_{t} \in C_{y}, W_{t / 2} \in A_{x}\right)=\mathbb{P}^{\nu_{y}}\left(W_{t} \in C_{x}, W_{t / 2} \in A_{x}\right),
$$

which can be bounded in exactly the same way as (6.19). Therefore, as $q$ is continuous it follows from (6.18), (6.19) and (6.1) that

$$
q(t, x, y) \leq 2 \sup _{x^{\prime}} \mathbb{P}^{x x^{\prime}}(S<t / 2) \sup _{z} q(t / 2, z, z) .
$$

The upper bounds in (a) and (b) now follow immediately from (6.20) on using the bounds given in Lemma 6.3 and Theorem 6.8.

We now turn to the lower bounds in (a) and (b). Using a standard chaining argument such as in the proof of Theorem 7.4 of [BB4], these can be proved once we establish the estimates

$$
\begin{aligned}
& q(t, x, y) \geq c_{9} t^{-d_{s} / 2}, \quad|x-y| \leq c_{10} t^{1 / d_{w}}, \quad t \geq 1, \\
& q(t, x, y) \geq c_{11} t^{-d / 2}, \quad|x-y| \leq c_{12} t^{1 / 2}, \quad t \leq 1 .
\end{aligned}
$$

(6.22) is proved in Proposition 6.2, so we just prove (6.21).

We deduce from the upper bound (6.16) that there exists $c_{13}$ such that

$$
q(t, x, x) \geq c_{13} t^{-d_{s} / 2}, \quad x \in \tilde{F}_{0}, \quad t \geq 1 ;
$$


the proof is as follows ( $c f$. Lemma 7.1 in [BB4]). By (5.19)

$$
\mathbb{P}^{\mathrm{P}}\left(\sigma_{r}(x) \leq t\right) \leq c_{14} \exp \left(-c_{15}\left(t_{F}^{r} t\right)^{-1 /\left(d_{w}-1\right)}\right)
$$

Pick $a$ such that $c_{14} \exp \left(-c_{15} a^{-1 /\left(d_{w}-1\right)}\right) \leq \frac{1}{2}$ and let $r=\left[\log (2 a / t) / \log t_{F}\right]$. Then

$$
\mathbb{P}^{x}\left(W_{s} \in D_{r}(x)\right) \geq \mathbb{P}^{x}\left(\sigma_{r}(x)>t / 2\right) \geq \frac{1}{2}
$$

and

$$
\mu_{0}\left(D_{r}(x)\right) \leq c_{16} m_{F}^{-r} \leq c_{17} t^{d_{s} / 2}
$$

By Cauchy-Schwarz,

$$
\begin{aligned}
1 / 4 & \leq\left[\mathbb{P}^{x}\left(W_{t / 2} \in D_{r}(x)\right)\right]^{2}=\left(\int_{D_{r}(x)} q(t / 2, x, y) \mu_{0}(d y)\right)^{2} \\
& \leq \mu_{0}\left(D_{r}(x)\right) \int_{D_{r}(x)} q(t / 2, x, y)^{2} \mu_{0}(d y) \\
& \leq \mu_{0}\left(D_{r}(x)\right) q(t, x, x) .
\end{aligned}
$$

(6.23) now follows immediately.

By (6.16) there exists $c_{18}$ such that

$$
q(t, x, y) \leq c_{18} t^{-d_{s} / 2}, \quad x, y \in \tilde{F}_{0}
$$

Now fix $t \geq 1$ and $x \in \tilde{F}_{0}$. Take $c_{19}=\left(c_{13} \wedge c_{18}\right) / 2$ and let $A=\{y: q(t / 2, x, y)>$ $\left.c_{19} t^{-d_{s} / 2}\right\}$. Then

$$
\begin{aligned}
c_{13} t^{-d_{s} / 2} & \leq q(t, x, x)=\int q(t / 2, x, y) q(t / 2, x, y) \mu_{0}(d y) \\
& \leq \int_{A} c_{18} t^{-d_{s} / 2} q(t / 2, x, y) \mu_{0}(d y)+\int_{A^{c}} c_{19} t^{-d_{s} / 2} q(t / 2, x, y) \mu_{0}(d y) .
\end{aligned}
$$

Therefore

$$
\begin{aligned}
c_{13} & \leq c_{18} \mathrm{P}^{x}\left(W_{t / 2} \in A\right)+c_{19} \mathrm{P}^{x}\left(W_{t / 2} \in A^{c}\right) \\
& =c_{19}+\left(c_{18}-c_{19}\right) \mathrm{P}^{x}\left(W_{t / 2} \in A\right) .
\end{aligned}
$$

So, if $c_{20}=\left(c_{13}-c_{19}\right) /\left(c_{18}-c_{19}\right)$, then $\mathrm{PP}^{x}\left(W_{t / 2} \in A\right) \geq c_{20}$.

Let $\varepsilon=c_{20} / 4$, and choose $m$ such that

$$
\mathbb{P}^{y}\left(\sigma_{m}(x) \geq t / 2\right)<\varepsilon, \quad y \in D_{m}(x) .
$$

By (6.9) this will hold if $m$ is chosen so that $t t_{F}^{m} \geq c_{21} \geq t t_{F}^{m-1}$, for a suitable $c_{21}$. Write $D=D_{m}(x)$. As $\varepsilon$ depends only on $d$ and $l_{F}$, by Theorem 3.25(b) there exists $\delta>0$, 
depending only on $d$ and $l_{F}$, such that if $|x-y| \leq \delta l_{F}^{-m}$, then there exist $\operatorname{RBM}\left(\tilde{F}_{0}\right), W^{x}$, $W^{y}$, starting at $x, y$, respectively, such that

$$
\mathbb{P}\left(T_{C}\left(W^{x}, W^{y}\right)<\mathcal{T}_{D}\left(W^{x}\right) \wedge \mathcal{T}_{D}\left(W^{y}\right)\right)>1-\varepsilon .
$$

Therefore, writing $T_{C}=T_{C}\left(W^{x}, W^{y}\right)$,

$$
\begin{aligned}
1-\varepsilon & <\mathbb{P}\left(T_{C}<\mathcal{T}_{D}\left(W^{x}\right) \wedge \mathcal{T}_{D}\left(W^{y}\right)\right) \\
& \leq \mathbb{P}\left(T_{C} \leq t / 2\right)+P\left(\mathcal{T}_{D}\left(W^{x}\right) \geq t / 2\right)+P\left(\mathcal{T}_{D}\left(W^{y}\right) \geq t / 2\right),
\end{aligned}
$$

so that

$$
\mathbb{P}\left(T_{C} \leq t / 2\right) \geq 1-3 \varepsilon .
$$

Thus

$$
\begin{aligned}
\mathbb{P}\left(W_{t / 2}^{y} \in A\right) & \geq \mathbb{P}\left(W_{t / 2}^{x} \in A, T_{C} \leq t / 2\right) \\
& \geq \mathbb{P}\left(W_{t / 2}^{x} \in A\right)-\mathbb{P}\left(T_{C}>t / 2\right) \\
& \geq c_{20}-3 \varepsilon=c_{20} / 4
\end{aligned}
$$

So if $|x-y| \leq \delta l_{F}^{-m}$, then

$$
\begin{aligned}
q(t, x, y) & \geq \int_{A} q(t / 2, x, z) q(t / 2, z, y) \mu_{0}(d z) \\
& \geq c_{19} t^{-d_{s} / 2} \mathrm{P}^{y}\left(W_{t / 2} \in A\right) \geq c_{19} c_{20} t^{-d_{s} / 2} / 4 .
\end{aligned}
$$

Since $l_{F}^{-m} \leq c_{21} 1^{1 / d_{w}}$ and $\delta$ depends only on $d$ and $l_{F}$, the estimate (6.21) follows.

Observe that Theorem 6.9 is the same as Theorem 1.4.

For $\lambda \geq 0$ let

$$
v^{\lambda}(x, y)=\int_{0}^{\infty} q(t, x, y) e^{-\lambda t} d t
$$

be the $\lambda$-potential kernel density for $W$. Let $v=v^{0} ; v$ is the Green function for $W$. Integrating the bounds in Theorem 6.9 we have:

Corollary 6.10 Let $d_{s}>2$. Then

$$
\begin{gathered}
c_{1}|x-y|^{d-2} \leq v(x, y) \leq c_{2}|x-y|^{d-2} \quad \text { if }|x-y| \leq 1, \\
c_{3}|x-y|^{d_{w}-d_{f}} \leq v(x, y) \leq c_{4}|x-y|^{d_{w}-d_{f}} \quad \text { if }|x-y| \geq 1 .
\end{gathered}
$$

Recall that for $n \geq 0$ the process $X^{n}$ on $\tilde{F}_{n}$ is given by

$$
X_{t}^{n}=l_{F}^{-n} W_{t\left(t_{F}^{n}\right)}, \quad t \geq 0 .
$$


Let $p_{n}(t, x, y)$ be the transition density of $X^{n}$ with respect to $\mu_{n}$. Then from (6.24) we have the scaling relation

$$
p_{n}(t, x, y)=m_{F}^{n} q\left(t_{F}^{n} t, l_{F}^{n} x, l_{F}^{n} y\right)
$$

Theorem 6.9(a) therefore implies:

Corollary 6.11 There exist $c_{1}, c_{2}, c_{3}, c_{4} \in(0, \infty)$ such that if $n \geq 0, x, y \in \tilde{F}_{n}$ and $t \geq t_{F}^{-n}$, $|x-y| \leq l_{F}^{n\left(d_{w}-1\right)} t$, then

$$
\begin{gathered}
c_{1} t^{-d_{s} / 2} \exp \left(-c_{2}\left(\frac{|x-y|^{d_{w}}}{t}\right)^{1 /\left(d_{w}-1\right)}\right) \\
\leq p_{n}(t, x, y) \leq c_{3} t^{-d_{s} / 2} \exp \left(-c_{4}\left(\frac{|x-y|^{d_{w}}}{t}\right)^{1 /\left(d_{w}-1\right)}\right) .
\end{gathered}
$$

Remark 6.12 Taking a limit along a subsequence shows that the density $p(t, x, y)$ of $X_{t}$ with respect to $\mu$ exists and gives the bounds in Theorem 1.3.

Define the $\lambda$-resolvent of $X$ by $U^{\lambda} f(x)=\mathbb{E}^{x} \int_{0}^{\infty} e^{-\lambda s} f\left(X_{s}\right) d s$ for $\lambda \geq 0$ and write $U$ for $U^{0}$. Set also

$$
u^{\lambda}(x, y)=\int_{0}^{\infty} e^{-\lambda t} p(t, x, y) d t
$$

and write $u(x, y)=u^{0}(x, y)$. Then $u^{\lambda}$ is the density of the $U^{\lambda}$, and $u$ is a Green function for $X$. The following bounds for $u^{\lambda}(x, y)$ are obtained by integrating those for $p(t, x, y)$; in (b) and (c) below one uses Laplace's method.

\section{Corollary 6.13}

(a) If $d_{s}>2$ then

$$
c_{1}|x-y|^{d_{w}-d_{f}} \leq u(x, y) \leq c_{2}|x-y|^{d_{w}-d_{f}}, \quad x, y \in \tilde{F}
$$

(b) If $d_{s}<2$ then for $\lambda>0, x, y \in \tilde{F}$,

$$
c_{3} \lambda^{d_{s} / 2-1} \exp \left(-c_{4} \lambda^{1 / d_{w}}|x-y|\right) \leq u^{\lambda}(x, y) \leq c_{5} \lambda^{d_{s} / 2-1} \exp \left(-c_{6} \lambda^{1 / d_{w}}|x-y|\right) .
$$

(c) If $d_{s}=2$ then writing $r=\lambda^{1 / d_{w}}|x-y|$,

$$
c_{7}\left(\log ^{+}(1 / r)+e^{-c_{8} r}\right) \leq u^{\lambda}(x, y) \leq c_{9}\left(\log ^{+}(1 / r)+e^{-c_{10} r}\right)
$$

Since there are only countably many GSCs, and (as far as we know) there is no simple expression for $d_{s}$, it is unlikely that any GSC actually satisfies case (c) above. However, we include it for completeness.

We now show that $p(t, x, y)$ is $C^{\infty}$ in $t$ and each of the partial derivatives is Hölder continuous in $x$ and $y$. Let $m<-2$ be fixed and let $\bar{P}_{t}$ be defined on $D_{m}(0)$ by

$$
\bar{P}_{t} f(x)=\mathbb{E}^{x}\left[f\left(X_{t}\right) ; t<\sigma_{m}(0)\right] .
$$


Let $\bar{p}(t, x, y)$ be the corresponding transition density for $X_{t}$ killed on exiting $D_{m}(0)$. Since $\bar{p}(t, x, y) \leq p(t, x, y)$, we have

$$
\bar{p}(t, x, y) \leq c_{1} t^{-d_{s} / 2}, \quad x, y \in D_{m}(0) \cap \tilde{F}, \quad t \geq 0 .
$$

Let $\bar{u}^{\lambda}(x, y)=\int_{0}^{\infty} e^{-\lambda t} \bar{p}(t, x, y) d t$ and let $\bar{U}^{\lambda}$ be the corresponding resolvent operator. Write $\bar{U}$ for $\bar{U}^{0}$.

\section{Proposition 6.14}

(a) There exist $c_{1}$ and $\beta$ not depending on $m$ such that if $f$ is bounded, then

$$
\left|\bar{U}^{\lambda} f(x)-\bar{U}^{\lambda} f(y)\right| \leq c_{1}\left(1+\lambda^{-1}\right)|x-y|^{\beta}\|f\|_{\infty}, \quad x, y \in D_{m+2}(0) \cap \tilde{F} .
$$

(b) $\bar{U}^{\lambda} f$ is continuous in $D_{m}(0) \cap \tilde{F}$ with a modulus of continuity that depends only on $\lambda, m$ and $\|f\|_{\infty}$.

Proof (a) Let $\bar{U}_{D_{n}\left(x_{0}\right)}$ be the Green operator for $X_{t}$ killed on exiting $D_{n}\left(x_{0}\right)$. If $x_{0} \in D_{m}(0)$ and $D_{n}\left(x_{0}\right) \cap \tilde{F} \subset D_{m}(0) \cap \tilde{F}$, then for bounded $h$

$$
\bar{U} h(x)=\bar{U}_{D_{n}\left(x_{0}\right)} h(x)+\mathbb{E}^{x} \bar{U} h\left(X_{\sigma_{n}\left(x_{0}\right)}\right), \quad x \in D_{n}\left(x_{0}\right) .
$$

The second term is harmonic in $D_{n+2}\left(x_{0}\right)$, and so by Theorem 5.9 there exist $c_{2}$ and $\alpha$ (independent of $m$ ) such that

$$
\left|\mathbb{E}^{\alpha} \bar{U} h\left(X_{\sigma_{n}\left(x_{0}\right)}\right)-\mathbb{E}^{y} \bar{U} h\left(X_{\sigma_{n}\left(x_{0}\right)}\right)\right| \leq c_{2}|x-y|^{\alpha} l_{F}^{n \alpha}\|\bar{U} h\|_{\infty}
$$

if $x, y \in D_{n+2}\left(x_{0}\right)$. On the other hand, by (5.20),

$$
\left.\| \bar{U}_{D_{n}\left(x_{0}\right)}\right)\left\|_{\infty} \leq c_{3} t_{F}^{-n}\right\| h \|_{\infty} .
$$

Taking $x=x_{0}$ we therefore deduce that if $n, y$ satisfy

$$
D_{n}(x) \cap \tilde{F} \subset D_{m}(0) \cap \tilde{F}, \quad y \in D_{n+2}(x)
$$

then

$$
\begin{aligned}
|\bar{U} h(x)-\bar{U} h(y)| & \leq 2 c_{3} t_{F}^{-n}\|h\|_{\infty}+c_{2}|x-y|^{\alpha} l_{F}^{n \alpha}\|\bar{U} h\|_{\infty}, \\
& \leq c_{4}\left(\|h\|_{\infty}+\|\bar{U} h\|_{\infty}\right)\left(t_{F}^{-n}+l_{F}^{-n}|x-y|^{\alpha}\right) .
\end{aligned}
$$

Now suppose $x \in D_{m+2}(0)$ and $|x-y| \leq l_{F}^{-2} / 2$. Then if $n \geq 0$, (6.30) is satisfied. Choosing $n$ be the greatest integer less than $\alpha \log |x-y|^{-1} /\left(\left(\alpha+d_{w}\right) \log l_{F}\right)$ it follows from (6.31) that

$$
|\bar{U} h(x)-\bar{U} h(y)| \leq c_{5}\left(\|h\|_{\infty}+\|\bar{U} h\|_{\infty}\right)|x-y|^{\beta},
$$

where $\beta=\alpha d_{w}\left(\alpha+d_{w}\right)^{-1}$. 
By the resolvent identity,

$$
\bar{U}^{\lambda} f=\bar{U}\left(f-\lambda \bar{U}^{\lambda} f\right)
$$

Set $h=f-\lambda \bar{U}^{\lambda} f$; as $\left\|\bar{U}^{\lambda} f\right\|_{\infty} \leq \lambda^{-1}\|f\|_{\infty}$, we deduce $\|h\|_{\infty} \leq 2\|f\|_{\infty}$. As $\bar{U} h=\bar{U}^{\lambda} f$, $\|\bar{U} h\|_{\infty} \leq \lambda^{-1}\|f\|_{\infty}$, and so $\|h\|_{\infty}+\|\bar{U} h\|_{\infty} \leq\left(2+\lambda^{-1}\right)\|f\|_{\infty}$. Therefore

$$
\left|\bar{U}^{\lambda} f(x)-\bar{U}^{\lambda} f(y)\right| \leq c_{6}\left(1+\lambda^{-1}\right)|x-y|^{\beta}\|f\|_{\infty} .
$$

Finally, if $|x-y| \leq 1$ then we can find a chain $x=x_{0}, x_{1} \cdots x_{k}=y$ with $\left|x_{i-1}-x_{i}\right| \leq l_{F}^{-2} / 2$ and $k \leq c_{7}\left(d, l_{F}\right)$. So, using the triangle inequality (a) follows.

(b) Let $A=\partial_{r} D_{m}(0)$ be the relative boundary of $D_{m}(0) \cap \tilde{F}$ in $\tilde{F}$. Write $D=D_{m}(0) \cap$ $\tilde{F}-A$. If $x \in D$ then there exists $n_{0} \geq 2$ such that $D_{n_{0}}(x) \cap \tilde{F} \subset D$. If $y \in D_{n_{0}+2}(x) \cap \tilde{F}$ and $y \neq x$ choose $n$ so that $y \in D_{2 n}(x)-D_{2 n+2}(x)$. Then (6.30) holds, and so by (6.31)

$$
\begin{aligned}
|\bar{U} h(x)-\bar{U} h(y)| & \leq c_{8}\left(\|h\|_{\infty}+\|\bar{U} h\|_{\infty}\right)\left(t_{F}^{-n}+l_{F}^{-n \alpha}\right) \\
& \leq c_{9}\left(\|h\|_{\infty}+\|\bar{U} h\|_{\infty}\right)|x-y|^{\gamma}
\end{aligned}
$$

for some $\gamma>0$. Thus $\bar{U} h$ is continuous on $D$.

We now show $\bar{U} h$ vanishes continuously on $A$. For $n \geq 1$ set $B_{n}=\bigcup_{z \in A} D_{n}(z) \cap \tilde{F}$, $b_{n}=\sup _{x \in B_{n}}|\bar{U} h(x)|$. Note that by (5.20) $b_{1} \leq c_{3} t_{F}^{-m}\|h\|_{\infty}$.

By Corollary 3.24, there exists $\delta$ depending only on $l_{F}, d$ such that

$$
\mathbb{P}^{x}\left(X_{\sigma_{n}(x)} \notin D_{m}(0)\right) \geq \delta, \quad x \in B_{n+2} .
$$

Since $\bar{U} h=0$ outside $D_{m}(0)$,

$$
\begin{aligned}
|\bar{U} h(x)| & \leq c_{3} t_{F}^{-n}\|h\|_{\infty}+\left|\mathbb{E}^{x} \bar{U} h\left(X_{\sigma_{n}(x)}\right)\right| \\
& \leq c_{3} t_{F}^{-n}\|h\|_{\infty}+(1-\delta) b_{n-2}, \quad x \in B_{n+2} .
\end{aligned}
$$

Thus

$$
b_{n+2} \leq c_{3} t_{F}^{-n}\|h\|_{\infty}+(1-\delta) b_{n} .
$$

Thus $b_{n} \rightarrow 0$ as $n \rightarrow \infty$. We therefore have that $\bar{U} h$ is continuous on $D_{m}(0) \cap \tilde{F}$ with a modulus of continuity depending on $m$ and $\|h\|_{\infty}$. Using the resolvent identity as in (a), we obtain (b).

Proposition 6.15 There exist reals $0 \leq \lambda_{1} \leq \lambda_{2} \leq \cdots$ and bounded continuous functions $\varphi_{i}$ on $D_{m}\left(x_{0}\right)$ such that

(a) the only subsequential limit point of $\left\{\lambda_{i}\right\}$ is $\infty$;

(b) the $\varphi_{i}$ form a complete orthonormal system for $L^{2}\left(D_{m}\left(x_{0}\right), \mu\right)$;

(c) we have the expansion

$$
\bar{p}(t, x, y)=\sum_{i=1}^{\infty} e^{-\lambda_{i} t} \varphi_{i}(x) \varphi_{i}(y)
$$

where the convergence is absolute and uniform for each $t>0$. 
Proof We first show that if $\bar{P}_{t} f=0$ a.e. for $f \in L^{2}$, then $f=0$ a.e. By [FOT], Lemma 1.4.3 the semigroup $\left(\bar{P}_{t}\right)$ is strongly continuous on $L^{2}(F, \mu)$, so that $\left\|\bar{P}_{t} f-f\right\|_{2} \rightarrow 0$ as $t \downarrow 0$. If $\bar{P}_{t} f=0$, then

$$
0=\int f\left(\bar{P}_{t} f\right)=\int\left(\bar{P}_{t / 2} f\right)\left(\bar{P}_{t / 2} f\right),
$$

or $\bar{P}_{t / 2} f=0$ a.e. By induction $\bar{P}_{t / 2^{n}} f=0$ a.e., and so $f=0$ a.e.

$\bar{p}(t, x, y)$ is symmetric and bounded. Since $\mu\left(D_{m}\left(x_{0}\right)\right)<\infty$, then

$$
\int_{D_{m}\left(x_{0}\right)} \int_{D_{m}\left(x_{0}\right)} \bar{p}(t, x, y)^{2} \mu(d x) \mu(d y)<\infty .
$$

By [RS-N], $\bar{P}_{t}$ is a self-adjoint completely continuous operator on $L^{2}$, and by the proofs in [Bas2, Sect. II.4], there exist $\tilde{\varphi}_{i}$ that form a complete orthonormal system of functions in $L^{2}$ with

$$
\bar{p}(t, x, y)=\sum_{i=1}^{\infty} e^{-\lambda_{i} t} \tilde{\varphi}_{i}(x) \tilde{\varphi}_{i}(y)
$$

for $\mu^{2}$ a.e. pair $(x, y)$. Moreover the convergence is absolute and takes place in $L^{\infty}\left(D_{m}\left(x_{0}\right) \times\right.$ $\left.D_{m}\left(x_{0}\right)\right)$, and (a) holds.

Next note $\bar{P}_{t} \tilde{\varphi}_{i}=e^{-\lambda_{i} t} \tilde{\varphi}_{i}$ a.e., so $\bar{U}^{\lambda} \tilde{\varphi}_{i}=\left(\lambda+\lambda_{i}\right)^{-1} \tilde{\varphi}_{i}$ a.e. Setting $\varphi_{i}=\left(\lambda+\lambda_{i}\right) \bar{U}^{\lambda} \tilde{\varphi}_{i}$, we have $\varphi_{i}=\tilde{\varphi}_{i}$ a.e., while by Proposition $6.14 \varphi_{i}$ is continuous.

If $s<t$,

$$
e^{\lambda_{i} t} \bar{P}_{t} \tilde{\varphi}_{i}(x)=e^{\lambda_{i} s} \bar{P}_{s}\left(e^{\lambda_{i}(t-s)} \bar{P}_{t-s} \tilde{\varphi}_{i}\right)(x)=e^{\lambda_{i} s} \bar{P}_{s} \tilde{\varphi}_{i}(x)
$$

because $e^{\lambda_{i}(t-s)} \bar{P}_{t-s} \tilde{\varphi}_{i}=\tilde{\varphi}_{i}$ a.e. and $\bar{P}_{s}$ has a density. It follows that for each $x$ and $i$, $e^{\lambda_{i} t} \bar{P}_{t} \tilde{\varphi}_{i}(x)$ is constant in $t$. Writing $w_{i}(x)$ for $e^{\lambda_{i} t} \bar{P}_{t} \tilde{\varphi}_{i}(x)$,

$$
\bar{U}^{\lambda} \tilde{\varphi}_{i}(x)=\int_{0}^{t} e^{-\lambda t} \bar{P}_{t} \tilde{\varphi}_{i}(x) d t=\int_{0}^{\infty} e^{-\lambda t} e^{-\lambda_{i} t} w_{i}(x) d t=\left(\lambda+\lambda_{i}\right)^{-1} w_{i}(x) .
$$

Hence

$$
e^{\lambda_{i} t} \bar{P}_{t} \tilde{\varphi}_{i}(x)=w_{i}(x)=\varphi_{i}(x)
$$

for all $t$ and $x$. Since $\int \tilde{\varphi}_{i}^{2}(x) \mu(d x)=1$, from Cauchy-Schwarz and Remark 6.12,

$$
\varphi_{i}(x)=e^{\lambda_{i} t} \bar{P}_{t} \tilde{\varphi}_{i}(x) \leq e^{\lambda_{i} t}\left(\int \tilde{\varphi}_{i}^{2}(y) \mu(d y)\right)^{\frac{1}{2}}\left(\int_{D_{m}\left(x_{0}\right)} \bar{p}(t, x, y)^{2} \mu(d y)\right)^{\frac{1}{2}},
$$

and so $\varphi_{i}$ is bounded in $x$.

Let

$$
r(t, x, y)=\sum_{i=1}^{\infty} e^{-\lambda_{i} t} \varphi_{i}(x) \varphi_{i}(y) .
$$

As the $\varphi_{i}$ are orthonormal, then

$$
\int r(t / 2, x, z) r(t / 2, z, y) \mu(d z)=\sum_{i=1}^{\infty} e^{-\lambda_{i} t / 2} e^{-\lambda_{i} t / 2} \varphi_{i}(x) \varphi_{i}(y)=r(t, x, y) .
$$


If $f=\sum a_{i} \varphi_{i} \in L^{2}$,

$$
\int f(y) r(t, x, y) \mu(d y)=\sum e^{-\lambda_{i} t} a_{i} \varphi_{i}(x)
$$

while

$$
\bar{P}_{t} f(x)=\sum a_{i} \bar{P}_{t} \tilde{\varphi}_{i}(x)=\sum a_{i} e^{-\lambda_{i} t} \varphi_{i}(x) .
$$

Thus $r(t, x, \cdot)$ is a transition density for $\bar{P}_{t}$ and $r(t, x, \cdot)=\bar{p}(t, x, \cdot)$ a.e. We then have by $(6.33)$ that

$$
\begin{aligned}
\bar{p}(t, x, y) & =\int \bar{p}(t / 2, x, z) \bar{p}(t / 2, z, y) \mu(d z) \\
& =\int r(t / 2, x, z) r(t / 2, z, y) \mu(d y) \\
& =r(t, x, y) .
\end{aligned}
$$

From [Bas2, Sect. II.4], the sum in (6.33) is absolutely and uniformly convergent; consequently $\bar{p}(t, x, y)=r(t, x, y)$ is jointly continuous in $x$ and $y$.

Fix $t_{0}>0$, write $\lambda=t_{0}^{-1}$, and let

$$
\begin{gathered}
s_{k}(x, y)=\sum_{i=1}^{\infty}\left(\lambda+\lambda_{i}\right)\left(-\lambda_{i}\right)^{k} e^{-\lambda_{i} t_{0}} \varphi_{i}(x) \varphi_{i}(y), \quad k=0,1,2, \ldots, \\
S_{k} f(x)=\int f(y) s_{k}(x, y) \mu(d y) .
\end{gathered}
$$

Proposition 6.16 There exist constants $c_{1}(k)$ independent of $m$ such that if $f \in L^{1}$ then

$$
\left\|S_{k} f\right\|_{\infty} \leq c_{1}(k) t_{0}^{-k-1-d_{s} / 2}\|f\|_{1} .
$$

Proof Note first that $\sup _{r \geq 0}(\lambda+r) r^{k} e^{-r t_{0} / 2}=c_{1}(k) t_{0}^{-k-1}$ for some function $c_{1}(k)$. So, using Cauchy-Schwarz,

$$
\begin{aligned}
\left|s_{k}(x, y)\right| & \leq \sum_{i=1}^{\infty}\left(\lambda+\lambda_{i}\right) \lambda_{i}^{k} e^{-\lambda_{i} t_{0}}\left|\varphi_{i}(x)\right|\left|\varphi_{i}(y)\right| \\
& \leq\left(\sum_{i=1}^{\infty}\left(\lambda+\lambda_{i}\right) \lambda_{i}^{k} e^{-\lambda_{i} t_{0}} \varphi_{i}(x)^{2}\right)^{1 / 2}\left(\sum_{i=1}^{\infty}\left(\lambda+\lambda_{i}\right) \lambda_{i}^{k} e^{-\lambda_{i} t_{0}} \varphi_{i}(y)^{2}\right)^{1 / 2} \\
& \leq \sup _{x}\left(\sup _{i}\left(\lambda+\lambda_{i}\right) \lambda_{i}^{k} e^{-\lambda_{i} t_{0} / 2} \sum_{i=1}^{\infty} e^{-\lambda_{i} t_{0} / 2} \varphi_{i}(x)^{2}\right) \\
& \leq c_{1}(k) t_{0}^{-k-1} \sup _{x} \bar{p}\left(t_{0} / 2, x, x\right) \\
& \leq c_{2}(k) t_{0}^{-k-1-d_{s} / 2} .
\end{aligned}
$$


The result is now immediate.

Let $\partial_{t}$ be an abbreviation for $\partial / \partial t$ and $\partial_{t}^{k}$ an abbreviation for $\partial^{k} / \partial t^{k}$.

Proposition 6.17 For each $k \geq 0, t>0, \partial_{t}^{k} \bar{p}(t, x, y)$ is continuous in $x$ and $y$ on $D_{m}(0) \cap \tilde{F}$. Further there exist constants $c_{1}(k), c_{2}(k), \beta$, not depending on $m$, such that

$$
\begin{gathered}
\left|\partial_{t}^{k} \bar{p}(t, x, y)\right| \leq c_{1}(k) t^{-k-d_{s} / 2}, \quad x, y \in D_{m}(0) \cap \tilde{F}, \\
\left|\partial_{t}^{k} p(t, x, y)-\partial_{t}^{k} p\left(t, x^{\prime}, y\right)\right| \leq c_{2}(k)(1+t)\left|x-x^{\prime}\right|^{\beta} t^{-k-1-d_{s} / 2} \\
\text { for } x, x^{\prime}, \quad y \in D_{m}(0) \cap \tilde{F}, \quad\left|x-x^{\prime}\right| \leq 1 .
\end{gathered}
$$

Proof Let $f(z)=\bar{p}(s, z, y)$. Then

$$
\bar{P}_{t_{0}} f(x)=\int \bar{p}\left(t_{0}, x, z\right) \bar{p}(s, z, y) \mu(d z)=\bar{p}\left(t_{0}+s, x, y\right) .
$$

Therefore, using the eigenvalue expansion,

$$
\partial_{t}^{k} \bar{p}\left(t_{0}+s, x, y\right)=\partial_{t}^{k} \bar{P}_{t_{0}} f(x)=\bar{U}^{\lambda} S_{k} f(x) .
$$

Thus $\partial_{t}^{k} \bar{p}\left(t_{0}+s, x, y\right)$ is continuous on $D_{m}(0)$ by Proposition 6.14(b). As $\|f\|_{1}=1$ we have, using Proposition 6.16,

$$
\begin{aligned}
\left|\partial_{t}^{k} \bar{p}\left(t_{0}+s, x, y\right)\right| & \leq\left\|\bar{U}^{\lambda} S_{k} f\right\|_{\infty} \\
& \leq \lambda^{-1}\left\|S_{k} f\right\|_{\infty} \\
& \leq \lambda^{-1} c_{3}(k) t_{0}^{-1-k-d_{s} / 2}
\end{aligned}
$$

If $x, x^{\prime} \in D_{m+2}(0) \cap \tilde{F}$ and $\left|x-x^{\prime}\right| \leq 1$ then by Proposition 6.14(a)

$$
\begin{aligned}
\left|\partial_{t}^{k} \bar{p}\left(t_{0}+s, x, y\right)-\partial_{t}^{k} \bar{p}\left(t_{0}+s, x^{\prime}, y\right)\right| & \leq\left|\bar{U}^{\lambda} S_{k} f(x)-\bar{U}^{\lambda} S_{k} f\left(x^{\prime}\right)\right| \\
& \leq c_{4}\left(1+\lambda^{-1}\right)\left|x-x^{\prime}\right|^{\beta}\left\|S_{k} f\right\|_{\infty} \\
& \leq c_{5}(k)\left(1+t_{0}\right)\left|x-x^{\prime}\right|^{\beta} t_{0}^{-1-k-d_{s} / 2} .
\end{aligned}
$$

The result follows immediately from these bounds.

Theorem 6.18 There exist constants $c_{1}(k), c_{2}(k)$, depending only on $k$, and $\beta>0$ such that for $x, x^{\prime}, y \in \tilde{F}, t>0, k \geq 0$

$$
\left|\partial_{t}^{k} p(t, x, y)\right| \leq c_{1}(k) t^{-k-d_{s} / 2}
$$

Writing $R=\left|x-x^{\prime}\right| t^{-1 / d_{w}}$,

$$
\left|\partial_{t}^{k} p(t, x, y)-\partial_{t}^{k} p\left(t, x^{\prime}, y\right)\right| \leq c_{2}(k) \max \left(R^{\beta}, R^{d_{w}}\right) t^{-k-d_{s} / 2} .
$$


Proof As the bounds in Proposition 6.17 are independent of $m$ it follows by the argument of [BB4, Sect. 4], that $\partial_{t}^{k} p(t, x, y)$ is continuous and satisfies the bounds (6.34) and (6.35). This proves (6.36).

To prove (6.37) we use weak scaling. Let $X_{t}^{\prime}=l_{F}^{n} X\left(t / t_{F}^{n}\right)$, and let $p^{\prime}(t, x, y)$ be the transition density of $X^{\prime}$. Then $p^{\prime}$ also satisfies (6.34) and (6.35), and

$$
p(t, x, y)=m_{F}^{n} p^{\prime}\left(t_{F}^{n} t, l_{F}^{n} x, l_{F}^{n} y\right)
$$

Write $\theta=l_{F}^{n}$; if $\theta\left|x-x^{\prime}\right| \leq 1$, then using (6.35) it follows that

$$
\left|\partial_{t}^{k} p(t, x, y)-\partial_{t}^{k} p\left(t, x^{\prime}, y\right)\right| \leq c_{3}(k)\left(\theta^{-d_{w}}+t\right) \theta^{\beta}\left|x-x^{\prime}\right|^{\beta} t^{-k-1-d_{s} / 2}
$$

Optimizing over $\theta \in\left\{l_{F}^{n}, n \in \mathbb{Z}\right\}$ we obtain (6.37).

Proof of Theorem 1.3 The continuity and symmetry of $p(t, x, y)$ follow from Propositions 6.15 and 6.17, while the bounds in (1.4) follow from Corollary 6.11.

Proof of Theorem 1.2 Combine Theorem 1.3 and Theorem 5.7.

Remark 6.19 We can use the spectral theorem to obtain an expansion of $p(t, \cdot, \cdot)$ on $\tilde{F} \times \tilde{F}$.

Proposition 6.20 Suppose $\bar{p}(t, x, y)=\bar{p}_{D_{0}\left(x_{0}\right)}(t, x, y)$ is the transition density for $X_{t}$ killed on exiting $D_{0}\left(x_{0}\right)$. Let $t_{0} \in\left[1, l_{F}^{d}\right]$ and $c_{1}>0$. Then there exists $c_{2}$ such that $\bar{p}(t, x, y) \geq c_{2} t_{0}^{-d_{s} / 2}$ if $|x-y| \leq c_{1} t_{0}^{1 / d_{w}}$ and $x, y \in D_{2}\left(x_{0}\right)$.

Proof We have $\bar{p}(t, x, y) \leq p(t, x, y) \leq c_{3} t^{-d_{s} / 2}$ by Theorem 1.3. Just as in the proof of (6.23) there exists $r>2$ such that

$$
\bar{p}(t, x, x) \geq c_{4} t^{-d_{s} / 2}
$$

provided $x \in D_{r}\left(x_{0}\right)$. Just as in the proof of (6.21), we have

$$
\bar{p}(t, x, y) \geq c_{5} t^{-d_{s} / 2}, \quad|x-y| \leq c_{6} t^{1 / d_{w}}, \quad x, y \in D_{r+2}\left(x_{0}\right) .
$$

Finally we obtain our proposition by using the chaining argument in the proof of Theorem 7.4 of [BB4].

Proposition 6.21 Suppose $\widehat{p}(t, x, y)=\widehat{p}_{D_{0}\left(x_{0}\right)}(t, x, y)$ is the transition density for $X_{t}$ with reflection on $\partial D_{0}\left(x_{0}\right)$. There exists $c_{1}$ such that

$$
\widehat{p}(t, x, y) \geq c_{1} t^{-d_{s} / 2}, \quad t \geq 1
$$

Proof The proof is very similar to that for the unreflected process. In view of (5.26), if $x \in D_{r+2}(y)$, then $D_{r+4}(y) \subseteq D_{r}(x)$ and

$$
\mathbb{E}^{x} \sigma_{r+4}(y) \leq \mathbb{E}^{x} \sigma_{r}(x) \leq c_{2} t_{F}^{-r} .
$$


If $x \notin D_{r+2}(y)$, then $\mathbb{E}^{x} \sigma_{r+4}(y)=0$. Therefore,

$$
E^{x} \sigma_{r}(y) \leq c_{2} t_{F}^{-(r-4)} \leq c_{3} t_{F}^{-r}, \quad x, y \in F .
$$

$\sigma_{r}(y)$ is an additive functional for $X_{t}$ and by (6.38) and subadditivity (see [BK]),

$$
\mathbb{E}^{x} \sigma_{r}(y)^{p} \leq c_{4}(p) t_{F}^{-r p}, \quad p \geq 1, \quad x, y \in F .
$$

Proceeding just as in the proofs of Lemma 6.8, Proposition 6.7, and Theorem 6.8, we deduce

$$
\widehat{p}(t, x, y) \leq c_{5} t^{-d_{s} / 2} .
$$

Just as in the proof of (6.23),

$$
\widehat{p}(t, x, x) \geq c_{6} t^{-d_{s} / 2}, \quad x \in F,
$$

and as in the proof of $(6.21)$,

$$
\widehat{p}(t, x, y) \geq c_{7} t^{-d_{s} / 2}
$$

if $|x-y| \leq c_{8} t^{1 / d_{w}}$. An application of the chaining argument of Theorem 7.4 in [BB4] completes the proof.

\section{Inequalities}

In this section we apply the estimates obtained in Section 6 to deduce a number of Sobolev and related inequalities for the processes $X$ and $W$. Let $U$ be the potential operator for $X_{t}$, and $(\mathcal{E}, \mathcal{D}(\mathcal{E}))$ be the Dirichlet form defined in Section 5. From [CKS] and the upper bound $p(t, x, y) \leq c_{1} t^{-d_{s} / 2}$ given in Theorem 1.3 , we deduce immediately

Theorem 7.1 (Nash Inequality) There exists $c_{1}$ such that

$$
\|f\|_{2}^{2+4 / d_{s}} \leq c_{1} \mathcal{E}(f, f)\|f\|_{1}^{4 / d_{s}}, \quad f \in \mathcal{D}(\varepsilon) .
$$

Theorem 7.2 (Sobolev Inequality) Suppose $d_{s}>2$. Let $q=2 d_{s} /\left(d_{s}-2\right)$. Then there exists $c_{1}$ such that

$$
\|f\|_{q} \leq c_{1} \varepsilon(f, f)^{\frac{1}{2}}, \quad f \in \mathcal{D}(\varepsilon) .
$$

Thus Theorem 1.5 is proved.

Recall from Remark 5.11 the construction of Brownian motion on $D_{n}\left(x_{0}\right) \cap \tilde{F}$ with reflection on the boundaries of $D_{n}\left(x_{0}\right)$. Let $\widehat{p}(t, x, y)$ be the transition densities for this reflecting Brownian motion. Normalized Lebesgue measure on $D_{n}\left(x_{0}\right) \cap F_{m}$ is an invariant measure for the approximating processes $X_{t}^{m}$, and a limit argument shows that $\left.\mu\right|_{D_{n}\left(x_{0}\right)}$ is an invariant measure for reflecting Brownian motion. We write $\mathcal{E}_{D_{n}\left(x_{0}\right)}(f, f)$ for the Dirichlet form of this process, and let $\mathcal{D}_{n, x_{0}}$ be its domain. For $A \in \mathcal{B}(\tilde{F})$ let $f_{A}=\mu(A)^{-1} \int_{A} f d \mu$.

We now give a proof of the Poincare inequality, modeled after the proof in [SC].

Theorem 7.3 (Poincaré Inequality) There exists $c_{1}$ such that

$$
\int_{D_{n}\left(x_{0}\right)}\left|f-f_{D_{n}\left(x_{0}\right)}\right|^{2} \leq c_{1} l_{F}^{-n d_{w}} \mathcal{E}_{D_{n}\left(x_{0}\right)}(f, f), \quad f \in \mathcal{D}_{n, x_{0}} .
$$


Proof Let $t=l_{F}^{-n d_{w}}$. By Proposition 6.21 and weak scaling,

$$
\widehat{p}(t, x, y)=\widehat{p}_{D_{n}\left(x_{0}\right)}(t, x, y) \geq c_{2} l_{F}^{n d_{f}}
$$

Let $\widehat{P}_{t} f(x)=\int f(y) \widehat{p}(t, x, y) \mu(d y)$. Since $\left.\mu\right|_{D_{n}\left(x_{0}\right)}$ is an invariant measure for $\widehat{p}(t, x, y)$, then

$$
\int_{D_{n}\left(x_{0}\right)} \widehat{P}_{t} f(x) \mu(d x)=\iint \widehat{p}(t, x, y) f(y) 1_{D_{n}\left(x_{0}\right)}(x) \mu(d y) \mu(d x)=\int_{D_{n}\left(x_{0}\right)} f(y) \mu(d y) .
$$

Now from (7.1), for $x \in D_{n+1}\left(x_{0}\right)$,

$$
\widehat{P}_{t}\left(f-\widehat{P}_{t} f\right)^{2}(x) \geq c_{2} l_{F}^{n d_{f}} \int_{D_{n+1}\left(x_{0}\right)}\left|f-\widehat{P}_{t} f\right|^{2} \geq c_{2} l_{F}^{n d_{f}} \int_{D_{n+1}\left(x_{0}\right)}\left|f-f_{D_{n+1}\left(x_{0}\right)}\right|^{2}
$$

Integrating both sides over $D_{n+1}\left(x_{0}\right)$,

$$
\int_{D_{n+1}\left(x_{0}\right)} \widehat{P}_{t}\left(f-\widehat{P}_{t} f\right)^{2}(x) \mu(d x) \geq c_{3} \int_{D_{n+1}\left(x_{0}\right)}\left|f-f_{D_{n+1}\left(x_{0}\right)}\right|^{2}
$$

The left-hand side of (7.3) is equal to

$$
\begin{aligned}
\int_{D_{n}\left(x_{0}\right)} \widehat{P}_{t}\left(f^{2}\right)-\int_{D_{n}\left(x_{0}\right)}\left(\widehat{P}_{t} f\right)^{2} & =\int_{D_{n}\left(x_{0}\right)} f^{2}-\int_{D_{n}\left(x_{0}\right)}\left(\widehat{P}_{t} f\right)^{2} \\
& =-\int_{0}^{t} \int_{D_{n}\left(x_{0}\right)} \partial_{s}\left(\widehat{P}_{s} f\right)^{2} \mu(d y) d s \\
& =c_{4} t \mathcal{E}_{D_{n}\left(x_{0}\right)}(f, f) .
\end{aligned}
$$

The conclusion of Theorem 7.3 now follows by applying the techniques of Jerison [Je].

Suppose $d_{s}>2$. For $A \subset \tilde{F}$ define $C(A)$, the capacity of $A$, by

$$
C(A)=\sup \{\nu(A): U \nu \leq 1\}
$$

It is standard [FOT] that $C(A)$ can also be defined as

$$
C(A)=\inf \{\mathcal{E}(f, f): f=1 \text { on } A, f(x) \rightarrow 0 \text { as } x \rightarrow \infty\}
$$

if all points of $\partial A \cap \tilde{F}$ are regular for $A^{c}$.

Theorem 7.4 (Mass-Capacity Inequality) Let $d_{s}>2$. Then for $A \in \mathcal{B}(\tilde{F})$

$$
\mu(A) \leq c_{1} C(A)^{d_{s} /\left(d_{s}-2\right)}
$$


Proof Choose $n \in \mathbb{Z}$ such that $m_{F}^{-n} \leq \mu(A) \leq m_{F}^{-n+1}$. Write $\mu_{A}=\left.\mu\right|_{A}$. Then

$$
U\left(\mu_{A}\right)(x)=\int_{A} u(x, y) \mu(d y)=\int_{D_{n}(x)} u(x, y) \mu(d y)+\int_{A-D_{n}(x)} u(x, y) \mu(d y) .
$$

Now $\mu\left(D_{m}(x)-D_{m+1}(x)\right) \leq c_{2} m_{F}^{-m}$, while by Corollary 6.13 we have

$$
u(x, y) \leq c_{3}\left(l_{F}^{-m}\right)^{d_{w}-d_{f}}=c_{3} t_{F}^{-m} m_{F}^{m}, \quad y \in D_{n}(x)^{c} .
$$

Therefore

$$
\int_{D_{m}(x)-D_{m+1}(x)} u(x, y) \mu(d y) \leq c_{4} t_{F}^{-m}
$$

Summing over $m \geq n$ to bound the first term in (7.4) and using the estimate (7.5) in the second, we deduce that

$$
\begin{aligned}
U\left(\mu_{A}\right)(x) & \leq c_{5} t_{F}^{-n}+c_{3} t_{F}^{-n} m_{F}^{n} \mu_{A}(A) \\
& \leq c_{6} t_{F}^{-n} \leq c_{7} \mu(A)^{2 / d_{s}} .
\end{aligned}
$$

So if $\nu=\left(c_{7} \mu(A)^{2 / d_{s}}\right)^{-1} \mu_{A}$, then $U \nu \leq 1$, and therefore $C(A) \geq \nu(A)=c_{7}^{-1} \mu(A)^{1-2 / d_{s}}$.

We now proceed to obtain the Sobolev, mass-capacity, and Poincare inequalities for the pre-carpet. We start with the mass-capacity inequality. We let $C_{0}(A)$ denote the capacity of $A$ with respect to $\operatorname{RBM}\left(\tilde{F}_{0}\right)$. Recall that $\mu_{0}$ is Lebesgue measure restricted to $\tilde{F}_{0}$.

Theorem 7.5 Suppose $d_{s}>2$. There exists $c_{1}$ such that if $A \in \mathcal{B}\left(\tilde{F}_{0}\right)$ and $\mu_{0}(A) \geq 1$, then

$$
C_{0}(A) \geq c_{1} \mu_{0}(A)^{\left(d_{s}-2\right) / d_{s}} .
$$

Proof This is very similar to the proof of Theorem 7.4, except that we use Corollary 6.10 instead of Corollary 6.13. Let $A \subset \tilde{F}_{0}$, with $\mu_{0}(A) \geq 1$. Then choosing $n$ such that $m_{F}^{-n} \leq$ $\mu_{0}(A) \leq m_{F}^{-n+1}$, we have $n \leq 1$. Corollary 6.10 implies that

$$
\int_{D_{0}(x)} u(x, y) \mu(d y) \leq c_{2}
$$

and the remainder of the proof follows that of Theorem 7.4.

Remark 7.6 Write

$$
\mathcal{E}_{0}(f, f)=\frac{1}{2} \int_{\tilde{F}_{0}}|\nabla f|^{2} d x
$$

for the Dirichlet form associated with $W$. By [FOT, Sect. 2.2], the capacity of $A$ is equal to

$$
\inf \left\{\mathcal{E}_{0}(f, f): f \geq 1 \text { on } A, f \rightarrow 0 \text { as }\|x\|_{\infty} \rightarrow \infty\right\} .
$$

We now proceed to the Sobolev inequality.

Theorem 7.7 Suppose $d_{s}>2$. Let $q=d_{s} /\left(d_{s}-2\right)$. 
(a) There exists $c_{1}$ such that if $f$ is nonnegative and $r$ is such that $\mu_{0}(\{x: f(x)>r\}) \geq 1$, then

$$
\|f\|_{2 q} \leq c_{1} \varepsilon_{0}(f, f)^{1 / 2}+\left\|(f-r)^{+}\right\|_{2 q} .
$$

(b) There exists $c_{2}$ such that if $r$ and s are such that $\mu_{0}\left(\left\{x: f^{+}(x)>r\right\}\right) \geq 1$ and $\mu_{0}\left(\left\{x: f^{-}(x)>s\right\}\right) \geq 1$, then

$$
\|f\|_{2 q} \leq c_{2} \varepsilon_{0}(f, f)^{1 / 2}+\left\|\left(f^{+}-r\right)^{+}\right\|_{2 q}+\left\|\left(f^{-}-s\right)^{+}\right\|_{2 q} .
$$

Proof We have

$$
\|f\|_{2 q} \leq\|f \wedge r\|_{2 q}+\left\|(f-r)^{+}\right\|_{2 q} .
$$

So if we show

$$
\|f \wedge r\|_{2 q} \leq c_{1} \mathcal{E}_{0}(f \wedge r, \wedge r)^{1 / 2}
$$

(a) will follow since $\mathcal{E}_{0}(f \wedge r, f \wedge r) \leq \mathcal{E}_{0}(f, f)$.

We follow the proof of [Ma, pp. 110-112]. Let $\Phi(x, \xi)=|\xi|$ and $p=2$; then the $(p-\Phi)$-capacity of Maz'ja is, using Remark 7.6, the same as our capacity. Let $\mathcal{N}_{t}=\{x$ : $f(x) \wedge r>t\}$. By our assumption on $r, \mathcal{N}_{t}$ is either empty or $\mu_{0}\left(\mathcal{N}_{t}\right) \geq 1$. So using Theorem 7.5, $\mu_{0}\left(\mathcal{N}_{t}\right) \leq c_{3} C_{0}(A)^{q}$. We now obtain (a) by the proof in [Ma].

To prove (b), we write

$$
\|f\|_{2 q} \leq\left\|f^{+}\right\|_{2 q}+\left\|f^{-}\right\|_{2 q}
$$

and apply (a) to $f^{+}$and $f^{-}$, observing that $f^{+}=-(-f \wedge 0)$, so

$$
\mathcal{E}_{0}\left(f^{+}, f^{+}\right)=\varepsilon_{0}(-f \wedge 0,-f \wedge 0) \leq \varepsilon_{0}(-f,-f)=\varepsilon_{0}(f, f)
$$

and similarly for $\mathcal{E}_{0}\left(f^{-}, f^{-}\right)$.

Remark 7.8 The Sobolev inequality for $\mathcal{E}_{0}$ is more complicated than that for $\mathcal{E}$, as $q(t, x, x)$ behaves differently at 0 and $\infty$. We give here two other Sobolev inequalities for $\mathcal{E}_{0}$, both of which follow from the bounds in Theorem 6.9.

First, from [V2] we have

$$
\|f\|_{2 q} \leq c_{1}\left(\mathcal{E}_{0}(f, f)+\|f\|_{\infty}\right)
$$

We also have, from [Co],

$$
\left\|P_{1} f\right\|_{2 q} \leq c_{2} \mathcal{E}_{0}(f, f)
$$

Here $P_{1}$ can be replaced by other "regularizing" operators which smooth out short range fluctuations in $f$.

From [CKS, Theorem 2.9] we have the following conditional Nash inequality for $\mathcal{E}_{0}$.

Theorem 7.9 For each $c_{1}>0$ there exists $c_{2}$ such that

$$
\|f\|_{2}^{2+4 / d_{s}} \leq c_{2} \mathcal{E}_{0}(f, f)\|f\|_{1}^{4 / d_{s}} \quad \text { whenever } \quad \mathcal{E}_{0}(f, f) \leq c_{1}\|f\|_{1}^{2} .
$$


Finally we give a Poincaré inequality for $\tilde{F}_{0}$. Write

$$
\mathcal{E}_{0, D_{n}\left(x_{0}\right)}(f, f)=\int_{D_{n}\left(x_{0}\right) \cap \tilde{F}_{0}}|\nabla f(x)|^{2} d x .
$$

Theorem 7.10 There exists $c_{1}$ such that if $x_{0} \in \tilde{F}, n \leq 0$, then

$$
\int_{D_{n}\left(x_{0}\right)}\left|f-f_{D_{n}\left(x_{0}\right)}\right|^{2} \leq c_{1} t_{F}^{-n} \mathcal{E}_{0, D_{n}\left(x_{0}\right)}(f, f) .
$$

The proof is very similar to that of Theorem 7.3.

As remarked in the introduction, the bounds in Theorem 6.9 are sufficient to show that, while (by Theorem 4.3) an elliptic Harnack inequality holds for $\tilde{F}_{0}$, the usual type of parabolic Harnack inequality fails for any GSC for which $d_{w}>2$. (See Remark 5.4.)

Let $B \subset \mathbb{R}^{d}$ be open, and $0<t_{1}<t_{2} \leq \infty$. A function $u=u(t, x), t \in\left(t_{1}, t_{2}\right)$, $x \in B \cap \tilde{F}_{0}$, is parabolic on $\left(t_{1}, t_{2}\right) \times\left(B \cap \tilde{F}_{0}\right)$ if

$$
\frac{\partial u}{\partial t}=\frac{1}{2} \Delta u \quad \text { on } \quad\left(t_{1}, t_{2}\right) \times\left(B \cap \tilde{F}_{0}\right),
$$

and $\partial u / \partial n=0$ almost everywhere on $B \cap \partial \tilde{F}_{0}$. Let $x \in \tilde{F}_{0}, r>0, t>4 r^{2}$, and set

$$
\begin{gathered}
Q_{-}=\left[t-4 r^{2}, t-3 r^{2}\right] \times\left(B(x, 2 r) \cap \tilde{F}_{0}\right) \\
Q_{+}=\left[t-r^{2}, t\right] \times\left(B(x, r) \cap \tilde{F}_{0}\right) .
\end{gathered}
$$

The standard parabolic Harnack inequality (see for example [FS]) would assert that if $u$ is parabolic and non-negative then

$$
\sup _{Q_{-}} u \leq c_{1} \inf _{Q_{+}} u
$$

where the constant $c_{1}$ is independent of $x, r, t$ and $u$.

Proposition 7.11 Suppose the GSCF is such that $d_{w}>2$. Then the parabolic Harnack inequality (7.9) fails for $\tilde{F}_{0}$.

Proof Fix $x \in \tilde{F}_{0}$, and let $r>1$. Let $y \in \tilde{F}_{0}$ be chosen so that $|x-y|=r$, and let $u(s, z)=q(s, y, z)$. Take $t=5 r^{2}$. Then by (6.16)

$$
\sup _{Q_{-}} u \geq q\left(r^{2}, y, y\right) \geq c_{1} r^{-d_{s}},
$$

while (since $|x-y|=r<r^{2}<t$ )

$$
\begin{aligned}
\inf _{Q_{+}} u & \leq q\left(5 r^{2}, x, y\right) \\
& \leq c_{2} r^{-d_{s}} \exp \left(-c_{3}\left(r^{d_{w}} / 5 r^{2}\right)^{1 /\left(d_{w}-1\right)}\right) \\
& =c_{2} r^{-d_{s}} \exp \left(-c_{4} r^{\left(d_{w}-2\right) /\left(d_{w}-1\right)}\right) .
\end{aligned}
$$


Taking $r$ sufficiently large, (7.9) fails.

We can, however, give a different form for a parabolic Harnack inequality for $\tilde{F}_{0}$. Let $x \in \tilde{F}_{0}, r>1, t>4 r^{d_{w}}$, let $n$ be such that $l_{F}^{-n} \leq r<l_{F}^{-n+1}$, and set

$$
\begin{gathered}
R_{-}=\left[t-4 r^{d_{w}}, t-3 r^{d_{w}}\right] \times\left(D_{n}(x) \cap \tilde{F}_{0}\right), \\
R_{+}=\left[t-r^{d_{w}}, t\right] \times\left(D_{n+2}(x) \cap \tilde{F}_{0}\right) .
\end{gathered}
$$

Let $0 \leq t_{1}<t-4 r^{d_{w}}<t<t_{2}$, and $B \subset \mathbb{R}^{d}$ be an open set with $D_{n}(x) \subset B$. Let $u$ be non-negative and parabolic on $\left(t_{1}, t_{2}\right) \times\left(B \cap \tilde{F}_{0}\right)$.

Theorem 7.12 There exists a constant $c_{1}$, independent of $x, r, t, u$ such that

$$
\sup _{R_{-}} u \leq c_{1} \inf _{R_{+}} u
$$

The proof follows from the estimates on $q(t, x, y)$ in Theorem 6.9 by the same argument as that used in [FS].

We now discuss the functions in $\mathcal{D}(\mathcal{E})$ in a little more detail. If $f \in \mathcal{D}(\mathcal{E})$ then by [FOT, Lemma 1.3.4]

$$
\mathcal{E}(f, f)=\lim _{t \downarrow 0} \mathcal{E}_{t}(f, f)
$$

where

$$
\mathcal{E}_{t}(f, f)=t^{-1}\left(\left(I-P_{t}\right) f, f\right)
$$

Set

$$
\Gamma_{t}(f)(x)=(2 t)^{-1} \int p_{t}(x, y)(f(x)-f(y))^{2} \mu(d y)
$$

Then

$$
\begin{aligned}
\int \Gamma_{t}(f)(x) \mu(d x) & =\frac{1}{2} t^{-1} \iint p_{t}(x, y)(f(x)-f(y))^{2} \mu(d y) \mu(d x) \\
& =t^{-1} \iint f(x) p_{t}(x, y)(f(x)-f(y)) \mu(d y) \mu(d x) \\
& =\mathcal{E}_{t}(f, f)
\end{aligned}
$$

Proposition 7.13 Suppose the GSCF is such that $d_{w}>2$. Let $f \in C^{1}\left(\mathbb{R}^{d}\right)$, and suppose $g=\left.f\right|_{\tilde{F}} \in \mathcal{D}(\mathcal{E})$. Then $g$ is constant.

Proof Suppose $g$ is non-constant. Then we can find $x_{0} \in \tilde{F}$ such that $f$ is approximately linear (with non-zero gradient) in a neighbourhood of $x_{0}$. It follows that we can find sufficiently small constants $a_{0}, a_{1}, a_{2}, a_{3}$ such that whenever $x \in B_{0}=B\left(x_{0}, a_{0}\right)$ and $0<t<a_{1}$, there exists $y_{0} \in B\left(x, t^{1 / d_{w}}\right)$ such that

$$
|f(y)-f(x)| \geq a_{2} t^{1 / d_{w}} \quad \text { for } y \in B\left(y_{0}, a_{3} t^{1 / d_{w}}\right)
$$


So, writing $B_{1}=B\left(y_{0}, a_{3} t^{1 / d_{w}}\right)$, and $c_{1}, c_{2}$ for positive constants depending on the $a_{i}$,

$$
\begin{aligned}
\Gamma_{t}(f)(x) & \geq(2 t)^{-1} \int_{B_{1}} p_{t}(x, y)(f(x)-f(y))^{2} \mu(d y) \\
& \geq c_{1} t^{-1} \mu\left(B_{1}\right) t^{-d_{f} / d_{w}} t^{2 / d_{w}} \geq c_{2} t^{\left(2-d_{w}\right) / d_{w}} .
\end{aligned}
$$

Hence

$$
\varepsilon_{t}(f, f) \geq \int_{B_{0}} \Gamma_{t}(f)(x) \mu(d x) \geq c_{2} \mu\left(B_{0}\right) t^{\left(2-d_{w}\right) / d_{w}} .
$$

Hence $\lim _{t \downarrow 0} \mathcal{E}_{t}(f, f)=\infty$, so $f \notin \mathcal{D}(\mathcal{E})$.

Remark 7.14 For the Sierpinski gasket, see [BP, Corollary 9.2] (or [BST, Theorem 4.4] for an analytic proof) for an analogous result for $\mathcal{D}(\mathcal{L})$.

Remark 7.15 There are several recent papers on Sobolev inequalities and capacity on general metric spaces (including fractals) — see, for example, $[\mathrm{H} 1]$ and $[\mathrm{KM}]$. Specialized to a GSC $\tilde{F}$, Hajłasz [H1] defines the space $L^{1,2}(\tilde{F}, \mu)$ to be the set of $f: \tilde{F} \rightarrow \mathbb{R}$ such that there exists $E \subset \tilde{F}$ and $g \in L^{2}(\tilde{F}, \mu)$ such that for $x, y \in \tilde{F}-E$,

$$
|f(x)-f(y)| \leq|x-y|(g(x)+g(y)) .
$$

The norm $\|f\|_{L^{1,2}}$ is defined to be the infimum of $\|g\|_{2}$ for $g$ satisfying the above.

As an example of the kind of results that can be obtained, Hajłasz proves [H1, Lemma 2] the Poincaré inequality

$$
\int_{B}\left|f(x)-f_{B}\right|^{2} \leq c r^{2}\|f\|_{L^{1,2}},
$$

where $B=B(x, r) \cap \tilde{F}$. Thus this Poincaré inequality has the usual $r^{2}$ scaling, rather than the $r^{d_{w}}$ scaling of Theorem 7.3.

While the relation between these two approaches is not clear to us in general, we can remark that Hajłasz's space $L^{1,2}(\tilde{F}, \mu)$ is very different from $\mathcal{D}(\mathcal{E})$. For example, if $f \in$ $C_{K}^{1}\left(\mathbb{R}^{d}\right)$ and $f_{0}=\left.f\right|_{\tilde{F}}$ then $f_{0} \in L^{1,2}(\tilde{F}, \mu)$, while, by Proposition 7.13, $f_{0} \in \mathcal{D}(\mathcal{E})$ only if $f_{0}$ is constant.

\section{Properties of the Process}

In this section we list a number of properties of the processes $X$ and $W$.

\section{(a) Spectral Dimension}

Let $x_{0}$ be fixed and let $\bar{p}(t, x, y)$ be the transition densities of $X_{t}$ killed on exiting $D_{0}\left(x_{0}\right)$. By Proposition 6.15 we may write

$$
\bar{p}(t, x, y)=\sum_{i=1}^{\infty} e^{-\lambda_{i} t} \varphi_{i}(x) \varphi_{i}(y)
$$


for suitable eigenvalues $\lambda_{i}$ and eigenfunctions $\varphi_{i}$ (cf. [Bas2, Section II.4]). Just as in [BP, pp. 618-619],

$$
\lim _{\lambda \rightarrow \infty} \frac{\log \#\left\{\lambda_{j}: \lambda_{j} \leq \lambda\right\}}{\log \lambda}=d_{s}
$$

which says that $d_{s}$ agrees with what is known in the mathematical physics literature as the spectral dimension.

\section{(b) Transience, Recurrence and Regularity of Points}

Let $T_{x}=\inf \left\{t>0: X_{t}=x\right\}$.

Theorem 8.1 $X$ is transient if and only if $d_{s}>2$. Points are regular for themselves if and only if $d_{s}<2$. More precisely, if $x, y \in \tilde{F}$ then:

(a) if $d_{s}<2$, then $\mathbb{P}^{x}\left(T_{y}<\infty\right)=1$ and $\mathbb{P}^{x}\left(T_{x}=0\right)=1$,

(b) if $d_{s}=2$, then $\mathbb{P}^{x}\left(T_{y}<\infty\right)=0, \mathbb{P}^{x}\left(T_{x}=0\right)=0$, and $\mathbb{P}^{x}\left(\mathcal{T}_{D_{n}(y)}<\infty\right)=1$ for all $n$,

(c) if $d_{s}>2$, then $\mathbb{P}^{x}\left(T_{y}<\infty\right)=0, \mathbb{P}^{x}\left(T_{x}=0\right)=0$, and $\mathbb{P}^{x}\left(\lim _{t \rightarrow \infty}\left|X_{t}-y\right|=\infty\right)=1$.

Proof (a) may be proved as in [BB4, Theorem 8.1].

(b) Let $\lambda>0$. By Corollary 6.13 (c) $u^{\lambda}(x, y)<\infty$ for $x \neq y$, while $u^{\lambda}(x, x)=\infty$ for all $x$. Since $u^{\lambda}\left(X_{t}, y\right)$ is a non-negative $\mathbb{P}^{x}$-supermartingale, it follows that $X_{t}$ cannot hit $y$.

Since $X_{t}$ has a density, $\mathbb{E}^{x} \int_{0}^{\sigma_{m}\left(x_{0}\right)} 1_{\{x\}}\left(X_{s}\right) d s \leq \int_{0}^{\infty} p(s, x, x) d s=0$, or $X_{t}$ leaves $\{x\}$ immediately. By the preceding paragraph and the strong Markov property, $X_{t}$ never returns to $x$.

Let $\bar{u}(x, y)$ be the Green function for $X_{t}$ killed on exiting $D_{0}(y)$. We observe that there exist $c_{1}$ and $c_{2}$ such that

$$
\bar{u}(x, y)=\int_{0}^{\infty} \bar{p}(t, x, y) d t \geq c_{1} \int_{c_{2}|x-y| d_{w}}^{1} t^{-d_{s} / 2} d t \geq-c_{3} \log |x-y|, \quad x, y \in D_{2}(y) .
$$

From (2.12), Theorem 6.9, and integration we obtain $\bar{u}(x, y) \leq-c_{4} \log |x-y|$. Since $\bar{u}\left(X_{t \wedge \sigma_{n+m}(y)}, y\right)$ is a martingale and $\bar{u}(x, y)=0$ if $x \in \partial D_{0}(y)$, this martingale is a timechange of a one-dimensional Brownian motion. Since $\sigma_{0}(y)$ has finite expectation, it is finite almost surely, and we conclude that

$$
\mathbb{P P}_{F}^{l_{F}^{m-n} x}\left(\mathcal{T}_{D_{n+m}(y)}<\sigma_{0}(y)\right) \rightarrow 1 \quad \text { as } \quad m \rightarrow-\infty
$$

By weak scaling, $\mathrm{P}^{x x}\left(\mathcal{T}_{D_{n}(y)}<\sigma_{m}(y)\right) \rightarrow 1$ as $m \rightarrow-\infty$, which completes the proof of (b).

(c) The first two assertions are proved as in (b). $u\left(X_{t}, y\right)$ is a nonnegative supermartingale, hence it converges a.s. It is clear that the only possible limit value is 0 . Therefore $\left|X_{t}\right| \rightarrow \infty$, a.s. 


\section{(c) Modulus of Continuity}

There exists $c_{1}$ such that

$$
\lim _{\delta \rightarrow 0} \sup _{\substack{\leq \leq s, t \leq T \\|s-t| \leq \delta}} \frac{\left|X_{t}-X_{s}\right|}{|s-t|^{1 / d_{w}}(\log (1 /|s-t|))^{\left(d_{w}-1\right) / d_{w}}} \leq c_{1}, \quad \text { a.s. }
$$

The proof is the same as that of the corresponding result for the Sierpinski gasket in [BP].

\section{(d) Occupation Times}

Proposition 8.2 Suppose $d_{s}>2$ and let

$$
A_{t}(r)=\sup _{x \in F_{0}} \int_{0}^{t} 1_{B(x, r)}\left(X_{s}\right) d s .
$$

Then there exists $c_{1}<\infty$ such that

$$
\limsup _{r \rightarrow 0} \frac{A_{\infty}(r)}{r^{d_{w}} \log (1 / r)} \leq c_{1}
$$

Proof Let $C_{t}(x, r)=\int_{0}^{t} 1_{B(x, r)}\left(X_{s}\right) d s$. By Corollary 6.10

$$
\sup _{y} \mathbb{E}^{y} C_{\infty}(x, r) \leq \sup _{y} \int_{B(x, r)}|z-y|^{d_{w}-d_{f}} \mu(d z) \leq c_{2} r^{d_{w}} .
$$

Since $C_{t}(x, r)$ is an additive functional, by subadditivity (see [BK]), there exist $c_{3}$ and $c_{4}$ such that

$$
\sup _{y} \mathbb{E}^{y} \exp \left(c_{3} C_{\infty}(x, r) / r^{d_{w}}\right) \leq c_{4}
$$

So

$$
\mathbb{P}^{\mathrm{p}}\left(C_{\infty}(x, r)>\lambda r^{d_{w}}\right) \leq c_{5} e^{-c_{6} \lambda} .
$$

There exist $M=c_{7} r^{-d}$ balls of radius $4 r$ such that if $x \in[0,1]^{d}$, then $B(x, r)$ is contained in one of these $M$ balls. Then

$$
\mathbb{P}^{y}\left(A_{\infty}(r)>c_{8} \log (1 / r) r^{d_{w}}\right) \leq c_{5} c_{7} r^{-d} e^{-c_{6} c_{8} \log (1 / r)} .
$$

If we choose $c_{8}$ sufficiently large (but depending only on $c_{6}$ and $d$ ), then

$$
\mathbb{P}^{y}\left(A_{\infty}(r) \geq c_{8} \log (1 / r) r^{d_{w}}\right) \leq r^{-2} .
$$

We apply this estimate for $r_{j}=2^{-j}, j=1,2, \ldots$ By the Borel-Cantelli lemma,

$$
\mathbb{P}^{y}\left(A_{\infty}\left(r_{j}\right) \geq c_{8} \log \left(1 / r_{j}\right) r_{j}^{d_{w}} \text { i.o. }\right)=0 .
$$

Since $A_{\infty}(r)$ increases in $r$ as does $\log (1 / r) r^{d_{w}}$ for $r$ small, while $\log (1 / 2 r)(2 r)^{d_{w}}$ is bounded by a constant times $\log (1 / r) r^{d_{w}}$, the proposition follows. 


\section{(e) Local Times}

If $d_{s}<2$, the argument of [BB4] shows $X_{t}$ has a jointly continuous local time. If $d_{s} \geq 2$, Theorem 8.1 shows that a point $y$ is never hit, so a local time at $y$ cannot exist.

\section{(f) Hausdorff Dimension of the Range}

Proposition 8.3 Suppose $d_{s}>2$. If $R(\omega)=\left\{X_{t}(\omega): 0 \leq t \leq 1\right\}$, then with probability one the Hausdorff dimension of $R(\omega)$ is $d_{w}$.

This provides some justification for calling $d_{w}$ the "dimension" of the walk. (See $[\mathrm{AO}]$, [RT].) If $d_{s}<2$, then the continuity of the local times of $X$ implies that the range of $X_{t}$ includes an open subset of $F$, and hence the Hausdorff dimension of the range is $d_{f}$.

Proof We first show the dimension of $R(\omega)$ is less than or equal to $d_{w}$. Let $\varepsilon>0$. By the modulus of continuity result in (c), there exists $M$ (depending on $\omega$ ) such that

$$
\left|X_{t}-X_{s}\right| \leq M|s-t|^{1 /\left(d_{w}+\varepsilon\right)}
$$

if $0 \leq s, t \leq 1$. Let $j$ be an integer, $\delta=1 /(j+1)$, and $t_{i}=i \delta, i=0,1, \ldots, j$. Then the set of balls $B\left(X_{t_{i}}(\omega), M \delta^{1 /\left(d_{w}+\varepsilon\right)}\right)$ covers $R(\omega)$. We also have

$$
\sum_{i=0}^{j}\left(M \delta^{1 /\left(d_{w}+\varepsilon\right)}\right)^{d_{w}+\varepsilon}=M^{d_{w}+\varepsilon} \sum_{i=0}^{j} \delta=M^{d_{w}+\varepsilon}<\infty
$$

for all $j$. This implies that the dimension of $R(\omega)$ does not exceed $d_{w}+\varepsilon$, and since $\varepsilon$ is arbitrary, $\operatorname{dim} R(\omega) \leq d_{w}$.

Next we show that $\operatorname{dim} R(\omega)$ is at least $d_{w}$. By Proposition 8.2 there exists $M^{\prime}$ (depending on $\omega)$ such that $A_{1}(r)(\omega) \leq M^{\prime} r^{d_{w}-\varepsilon}$ if $r \leq 1$. Suppose there exists a sequence of balls $B\left(x_{1}, r_{1}\right), B\left(x_{2}, r_{2}\right), \ldots$ that cover $R(\omega)$ with $\sup _{i} r_{i} \leq \frac{1}{2}$ and $\sum_{i} r_{i}^{d_{w}-2 \varepsilon} \leq 1 / M^{\prime}$. We have

$$
\int_{0}^{1} 1_{B\left(x_{i}, r_{i}\right)}\left(X_{s}\right) d s \leq A_{1}\left(r_{i}\right) \leq M^{\prime} r_{i}^{d_{w}-\varepsilon}
$$

Summing over $i$,

$$
1=\int_{0}^{1} 1_{R(\omega)}\left(X_{s}\right) d s \leq M^{\prime} \sum_{i} r_{i}^{d_{w}-\varepsilon} \leq\left(\frac{1}{2}\right)^{\varepsilon} M^{\prime} \sum_{i} r_{i}^{d_{w}-2 \varepsilon} \leq\left(\frac{1}{2}\right)^{\varepsilon},
$$

a contradiction. Therefore there cannot exist such a sequence of balls, and so $\operatorname{dim} R(\omega) \geq$ $d_{w}-2 \varepsilon$. However, $\varepsilon$ is arbitrary.

\section{(g) Self-Intersections}

By $[\mathrm{Ro}]$ and $[\mathrm{FiS}], X_{t}$ intersects itself if and only if

$$
\int_{D_{1}(x)}\left(u^{1}(x, y)\right)^{2} \mu(d y)<\infty
$$


where $u^{1}$ is the 1 -resolvent for $X_{t}$. Note that

$$
\int_{D_{1}(x)}\left(u^{1}(x, y)\right)^{2} \mu(d y)=\sum_{i=1}^{\infty} \int_{D_{i}(x)-D_{i+1}(x)}\left(u^{1}(x, y)\right)^{2} \mu(d y) .
$$

$\mu\left(D_{i}(x)\right) \leq c_{1} l_{F}^{-i d_{f}}$ and it is easy to see that $\mu\left(D_{i}(x)-D_{i+1}(x)\right) \geq c_{2} l_{F}^{-i d_{f}}$. Using the estimates for $u^{1}(x, y)$, which are obtained similarly to those for $u(x, y)$, it follows that $X_{t}$ has self-intersections if and only if

$$
\sum_{i=1}^{\infty}\left[\left(l_{F}^{-i}\right)^{d_{w}-d_{f}}\right]^{2} l_{F}^{-i d_{f}}<\infty
$$

that is, if $2\left(d_{w}-d_{f}\right)+d_{f}>0$, or if $d_{s}=2 d_{f} / d_{w}<4$.

\section{(h) Zero-One Law}

An event $A$ is a tail event if it is in $\sigma\left(X_{u} ; u \geq t\right)$ for all $t>0$. We prove that the tail $\sigma$-field is trivial.

Theorem 8.4 Suppose $A$ is a tail event. Then either $\mathbb{P}^{x}(A)$ is 0 for all $x$ or else it is 1 for all $x$.

Proof Let $\varepsilon>0$ and fix $x_{0} \in \tilde{F}$. By the martingale convergence theorem, $\mathbb{E}^{x_{0}}\left[1_{A} \mid \mathcal{F}_{t}\right] \rightarrow 1_{A}$ almost surely as $t \rightarrow \infty$. Choose $t_{0}$ large enough so that

$$
\mathbb{E}^{x_{0}}\left|\mathbb{E}^{x_{0}}\left[1_{A} \mid \mathcal{F}_{t_{0}}\right]-1_{A}\right|<\varepsilon
$$

Write $Y$ for $\mathbb{E}^{x_{0}}\left[1_{A} \mid \mathcal{F}_{t_{0}}\right]$. Using ${ }^{\prime}(5.21)$, choose $M$ large so that

$$
\mathbb{P}^{x_{0}}\left(\sup _{s \leq t_{0}}\left|X_{s}-x_{0}\right|>M t_{0}^{1 / d_{w}}\right)<\varepsilon
$$

For each $t$, by Theorem 6.18 we have the continuity of $P_{t} f(x)$ in $x$ with a modulus depending only on $t$ and $\|f\|_{\infty}$. We use scaling and choose $t_{1}$ large so that

$$
\left|P_{t_{1}} f(x)-P_{t_{1}} f\left(x_{0}\right)\right|<\varepsilon\|f\|_{\infty}, \quad\left|x-x_{0}\right| \leq M t_{0}^{1 / d_{w}} .
$$

We note

$$
\left|\mathbb{P}^{x_{0}}(A)-\mathbb{E}^{x_{0}}(Y ; A)\right|=\left|\mathbb{E}^{x_{0}}\left(1_{A} ; A\right)-\mathbb{E}^{x_{0}}(Y ; A)\right|<\varepsilon
$$

Since $A$ is a tail event, there exists $C$ such that $A=C \circ \theta_{t_{0}+t_{1}}$. Let $f(z)=\mathbb{P} z(C)$. By the Markov property at time $t_{1}$,

$$
\mathbb{E}^{w}\left(1_{C} \circ \theta_{t_{1}}\right)=\mathbb{E}^{w} \mathbb{E}^{X\left(t_{1}\right)} 1_{C}=\mathbb{E}^{w} f\left(X_{t_{1}}\right)=P_{t_{1}} f(w) .
$$


By the Markov property at time $t_{0}$ and (8.6),

$$
\mathbb{E}^{x_{0}}(Y ; A)=\mathbb{E}^{x_{0}}\left[Y \mathbb{E}^{X\left(t_{0}\right)}\left(1_{C} \circ \theta_{t_{1}}\right)\right]=\mathbb{E}^{x_{0}}\left[Y P_{t_{1}} f\left(X_{t_{0}}\right)\right]
$$

while

$$
\mathbb{P}^{x_{0}}(A)=\mathbb{E}^{x_{0}} 1_{A}=\mathbb{E}^{x_{0}} \mathbb{E}^{X\left(t_{0}\right)}\left(1_{C} \circ \theta_{t_{1}}\right)=\mathbb{E}^{x_{0}}\left[P_{t_{1}} f\left(X_{t_{0}}\right)\right]
$$

$$
\text { If }\left|X_{t_{0}}-x_{0}\right| \leq M t_{0}^{1 / d_{w}} \text {, then }\left|P_{t_{1}} f\left(X_{t_{0}}\right)-P_{t_{1}} f\left(x_{0}\right)\right|<\varepsilon \text { by (8.4). Since }
$$

$$
\begin{aligned}
& \mathbb{E}^{x_{0}}\left[Y P_{t_{1}} f\left(X_{t_{0}}\right)\right]=\mathbb{E}^{x_{0}}\left[Y P_{t_{1}} f\left(X_{t_{0}}\right) ;\left|X_{t_{0}}-x_{0}\right| \leq M t_{0}^{1 / d_{w}}\right] \\
& +\mathbb{E}^{x_{0}}\left[Y P_{t_{1}} f\left(X_{t_{0}}\right) ;\left|X_{t_{0}}-x_{0}\right|>M t_{0}^{1 / d_{w}}\right] \\
& \left|\mathbb{E}^{x_{0}}\left[Y P_{t_{1}} f\left(X_{t_{0}}\right) ;\left|X_{t_{0}}-x_{0}\right| \leq M t_{0}^{1 / d_{w}}\right]-P_{t_{1}} f\left(x_{0}\right) \mathbb{E}^{x_{0}}\left[Y ;\left|X_{t_{0}}-x_{0}\right| \leq M t_{0}^{1 / d_{w}}\right]\right| \leq \varepsilon,
\end{aligned}
$$

and

$$
\mathbb{E}^{x_{0}}\left[Y ;\left|X_{t_{0}}-x_{0}\right| \leq M t_{0}^{1 / d_{w}}\right]=\mathbb{E}^{x_{0}} Y-\mathbb{E}^{x_{0}}\left[Y ;\left|X_{t_{0}}-x_{0}\right|>M t_{0}^{1 / d_{w}}\right],
$$

then

$$
\left|\mathbb{E}^{x_{0}}\left[Y P_{t_{1}} f\left(X_{t_{0}}\right)\right]-P_{t_{1}} f\left(x_{0}\right) \mathbb{E}^{x_{0}} Y\right| \leq 3 \varepsilon
$$

Similarly

$$
|| \mathbb{E}^{x_{0}} P_{t_{1}} f\left(X_{t_{0}}\right)-P_{t_{1}} f\left(x_{0}\right) \mid \leq 3 \varepsilon
$$

Combining (8.5), (8.7), (8.8), (8.9), and (8.10),

$$
\left|\mathbb{P}^{x_{0}}(A)-\mathbb{P}^{x_{0}}(A) \mathbb{E}^{x_{0}} Y\right| \leq 7 \varepsilon
$$

Using this and (8.2),

$$
\left|\mathrm{P}^{x_{0}}(A)-\mathrm{P}^{x_{0}}(A) \mathrm{P}^{x_{0}}(A)\right| \leq 10 \varepsilon .
$$

Since $\varepsilon$ is arbitrary, we deduce $\mathbb{P}^{x_{0}}(A)=\left[\mathbb{P}^{x_{0}}(A)\right]^{2}$, or $\mathbb{P}^{x_{0}}(A)$ is 0 or 1 . Since $\mathbb{P}^{x}(A)=$ $\mathbb{E}^{x} P_{t_{1}} f\left(X_{t_{0}}\right)=P_{t_{0}}\left(P_{t_{1}} f\right)(x)$ is continuous in $x$ and $\tilde{F}$ is connected, then $\mathbb{P}^{x}(A)$ is either identically 0 or identically 1 .

Remarks 8.5 1. An event $A$ is invariant if $A \circ \theta_{t}=A$ for all $A$. In particular, invariant events are in the tail $\sigma$-field, hence trivial. It follows that there are no nonconstant bounded harmonic functions on $\tilde{F}$.

2. Since we have similar estimates for the transition densities of Brownian motion on the nested fractals, the same proof shows there is a zero-one law for (unbounded) nested fractals as well. 


\section{(i) Rate of Escape}

Proposition 8.6 Suppose $d_{s}>2$. Then

$$
\liminf _{t \rightarrow \infty} \frac{\left|X_{t}-x\right|}{t^{1 / d_{w}}(\log t)^{\gamma}}
$$

equals 0 with $\mathbb{P}^{x x}$ probability one if $\gamma>1 /\left(d_{w}-d_{f}\right)$ and equals $\infty$ with $\mathbb{P}^{x x}$ probability one if $\gamma<1 /\left(d_{w}-d_{f}\right)$.

Proof By Corollary 6.13(a) and the fact that $u\left(X_{t}, x\right)$ is a local martingale, there exist $0<$ $c_{1}<c_{2}<\infty$ such that

$$
c_{1}\left(1 \wedge(r /|y-x|)^{d_{w}-d_{f}}\right) \leq \mathbb{P}^{p} y\left(X_{t} \text { ever hits } B(x, r)\right) \leq c_{2}\left(1 \wedge(r /|y-x|)^{d_{w}-d_{f}}\right) .
$$

Using this, for any $q>1$ and any $z \in \tilde{F}$,

$$
\begin{aligned}
\mathbb{P}^{z}\left(\left|X_{t}-x\right| \leq r_{n} \text { for some } t>q^{n}\right) \\
\quad=\mathbb{E}^{z} \mathbb{P}^{X_{q^{n}}}\left(\left|X_{t}-x\right| \leq r_{n} \text { for some } t \geq 0\right) \\
\quad=\int \mathbb{P}^{y}\left(\left|X_{t}-x\right| \leq r_{n} \text { for some } t \geq 0\right) \mathbb{P}^{z}\left(X_{q^{n}} \in \mu(d y)\right) \\
\quad \leq c_{2} \int\left(\frac{r_{n}}{|y-x|} \wedge 1\right)^{d_{w}-d_{f}} p\left(q^{n}, z, y\right) \mu(d y) .
\end{aligned}
$$

Set $r_{n}=c_{3} q^{n / d_{w}}\left(\log q^{n}\right)^{-\gamma}$. If

$$
A_{n}=\left\{\left|X_{t}-x\right| \leq r_{n} \text { for some } t \geq q^{n}\right\},
$$

then

$$
\mathbb{P}^{z}\left(A_{n}\right) \leq c_{4}\left(\log q^{n}\right)^{-\gamma\left(d_{w}-d_{f}\right)}
$$

By the Borel-Cantelli lemma we see that if $\gamma>1 /\left(d_{w}-d_{f}\right)$, then $\mathbb{P}^{x}\left(A_{n}\right.$ i.o. $)=0$, no matter what $c_{3}$ is.

For the other direction

$$
\begin{aligned}
P^{x}\left(\left|X_{t}-x\right| \leq r_{n} \text { for some } t \in\left[q^{n}, q^{n+1}\right]\right)= & \mathbb{E}^{x} \mathbb{P}^{X_{q^{n}}}\left(\left|X_{t}-x\right| \leq r_{n} \text { for some } t \geq 0\right) \\
& -\mathbb{E}^{x} \mathbb{P}^{X_{q^{n+1}}}\left(\left|X_{t}-x\right| \leq r_{n} \text { for some } t \geq 0\right) .
\end{aligned}
$$

Let $r_{n}=c_{5} q^{n / d_{w}}\left(\log q^{n}\right)^{-\gamma}$. As above, the second term on the right is bounded by

$$
c_{6}\left(\log q^{n}\right)^{-\gamma\left(d_{w}-d_{f}\right)} q^{-1 / d_{w}} .
$$


On the other hand, the first term on the right is bounded below by

$$
\int \mathbb{P}^{y}\left(\left|X_{t}-x\right| \leq r_{n} \text { for some } t \geq 0\right) p\left(q^{n}, x, y\right) \mu(d y) \geq c_{7}\left(\log q^{n}\right)^{-\gamma\left(d_{w}-d_{f}\right)}
$$

Set $B_{n}=\left\{\left|X_{t}-x\right| \leq r_{n}\right.$ for some $\left.t \in\left[q^{n}, q^{n+1}\right]\right\}$. Then provided $q$ is sufficiently large,

$$
\mathbb{P}^{x}\left(B_{n}\right) \geq c_{8}\left(\log q^{n}\right)^{-\gamma\left(d_{w}-d_{f}\right)}
$$

If $\gamma<1 /\left(d_{w}-d_{f}\right)$, then $\sum \mathbb{P}^{x}\left(B_{n}\right)=\infty$.

Let $\varepsilon>0$. If $j \geq i+2$, then

$$
\begin{aligned}
\mathbb{P}^{x}\left(B_{i} \cap B_{j}\right) & =\mathbb{E}^{x}\left[\mathbb{P}^{X}\left(q^{i+1}\right)\left(\left|X_{t}-x\right| \leq r_{j} \text { for some } t \in\left[q^{j}-q^{i+1}, q^{j+1}-q^{i+1}\right]\right) ; B_{i}\right] \\
& \leq \mathbb{E}^{x}\left[\sup _{y} \mathbb{P}^{y}\left(\left|X_{t}-x\right| \leq r_{j} \text { for some } t \geq q^{j}-q^{i+1}\right) ; B_{i}\right] .
\end{aligned}
$$

As in the proof of (8.11), the right hand side is at most $c_{9} \mathrm{P}^{\mathrm{P} x}\left(B_{i}\right)\left(\log q^{j}\right)^{-\gamma\left(d_{w}-d_{f}\right)}$. Using (8.12), this in turn is bounded by $c_{10} \mathrm{P}^{x}\left(B_{i}\right) \mathrm{P}^{x}\left(B_{j}\right)$, so

$$
\mathbb{P}^{x}\left(B_{i} \cap B_{j}\right) \leq c_{10} \mathbb{P}^{x}\left(B_{i}\right) \mathbb{P}^{x}\left(B_{j}\right) .
$$

By a Borel-Cantelli lemma for dependent events (see, e.g., [Bas2, Prop. II.5.14]), $\mathbb{P}^{x}\left(B_{n}\right.$ i.o. $)>0$. The result now follows from Theorem 8.4 , the zero-one law.

\section{(j) Process on the Pre-Carpet}

The properties of $X$ above which relate to large scale behavior of the process have analogues for the process $W$ on $\tilde{F}_{0}$, with almost identical proofs. We summarize some of them in the following theorem.

\section{Theorem 8.7}

(a) If $d_{s} \leq 2$ then $\mathbb{P}^{\mathrm{P} x}\left(\mathcal{T}_{D_{n}(y)}^{W}<\infty\right)=1$ for all $x, y \in \tilde{F}_{0}$, so that $W$ is neighborhoodrecurrent.

(b) If $d_{s}>2$ then $W$ is transient, and

$$
\liminf _{t \rightarrow \infty} \frac{\left|W_{t}-x\right|}{t^{1 / d_{w}}(\log t)^{\gamma}}
$$

equals 0 with probability one if $\gamma>1 /\left(d_{w}-d_{f}\right)$ and equals $\infty$ with probability one if $\gamma<1 /\left(d_{w}-d_{f}\right)$.

(c) The tail $\sigma$-field for $W$ is trivial. 


\section{Examples and Open Problems}

\section{Examples}

We have already, in Example 5.4, defined the the carpet $\operatorname{SC}(a, b, d)$, where $a, b, d$ are integers, $d \geq 2$, and $1 \leq b<a$. Note that the symmetry assumption Hypothesis 2.1 (H1) implies that $a=b(\bmod 2)$, and that therefore $b \leq a-2$. Recall that for $\operatorname{SC}(a, b, d)$, $m_{F}=a^{d}-b^{d}$, and that

$$
\frac{a-b}{a^{d-1}}+\frac{b}{a^{d-1}-b^{d-1}} \leq \rho_{F} \leq \frac{a}{a^{d-1}-b^{d-1}} .
$$

Since $b^{d-1} \leq(a-2)^{d-1}<(a-2) a^{d-2}=a^{d-1}-2 a^{d-2}$, we have $\rho_{F}<\frac{1}{2} a^{3-d}$. In particular $\rho_{F}<1$ whenever $d>2$, so that $\operatorname{SC}(a, b, d)$ is transient for all $d \geq 3$. (This generalizes a result of Zhou $[Z])$. This result is not surprising, since all these spaces contain a copy of $\mathbb{R}_{+}^{2}$. $\mathrm{SC}(a, b, 2)$ is recurrent by Corollary 5.3 , since $d_{s} \leq d_{f}<2$.

To obtain a GSC in dimension 3 for which the processes $W$ or $X$ are recurrent, we need to ensure that $\tilde{F}$ does not contain a copy of $\mathbb{R}_{+}^{2}$. Take $d=3$, let $1 \leq b \leq a-2$, write $I_{a b}=((a-b) / 2 a,(a+b) / 2 a)$, let $\alpha(x)=\sum_{i=1}^{3} 1_{I_{a b}}\left(x_{i}\right)$, and let

$$
F_{1}=\left\{x \in F_{0}: \alpha(x) \leq 1\right\} .
$$

Call the resulting GSC a Menger Sponge (see [Man, p. 134]) or MS(a,b). For the basic $M S(3,1), F_{1}$ consists of $F_{0}$ with the central cube and the 6 adjacent cubes removed. (See [Man, p. 145] for an excellent picture.) Note that $m_{F}=a^{3}-\left(b^{3}+3 b^{2}(a-b)\right)$. Using shorts and cuts, as in Proposition 5.1, we can easily estimate $\rho_{F}$ for these sets: we obtain

$$
\frac{a-b}{a^{2}-b^{2}}+\frac{b}{(a-b)^{2}} \leq \rho_{F} \leq \frac{a}{(a-b)^{2}} .
$$

In particular, for $M S(3,1)$ we have $\frac{1}{2} \leq \rho_{F} \leq 3 / 4$, so that $M S(3,1)$ is transient. However, if $a=6$ and $b=4$ then we have $11 / 10 \leq \rho_{F} \leq 3 / 2$, so that $M S(6,4)$ is recurrent. On the other hand, as $m_{F}=56$, we have $d_{f}=\log 56 / \log 6 \approx 2.25>2$, so the family of Menger Sponges includes sets with Hausdorff dimension greater than 2, but spectral dimension less than 2.

\section{Open Problems}

We conclude this paper with some open questions concerning these processes. (See also the problems in [Bas3].)

1. Spectral Dimension Of the three "dimensions" mentioned in this paper, $d_{f}, d_{w}$, and $d_{s}$, the first is given explicitly in terms of $l_{F}$ and $m_{F}$. The other two are defined in terms of the resistance exponent $\rho_{F}$, which we only know how to define as a limit:

$$
\log \rho_{F}=\lim _{n \rightarrow \infty} \frac{\log R_{n}}{n} .
$$


Is there a more explicit equation which defines $\rho_{F}$ ? S. R. S. Varadhan has asked if $\rho_{F}$ could be defined by some kind of variational equation.

2. Uniqueness There are two different kinds of uniqueness we can consider. The first relates to the construction of $X$ and its law $\mathbb{P}^{x}$ given here. In Section 5 we stated that the set of laws of the approximating processes $\left\{\mathbb{P}_{n}^{x}, n \geq 0\right\}$ was weakly compact, and so has limit points. Can one show that $P_{n}^{x}$ converges weakly to $\mathbb{P}^{x}$, that is, that the limit is unique? If so, then one would immediately have that the resulting process was self-similar.

A second kind of uniqueness is given by our conjecture that the process constructed in this paper is (up to a deterministic time change) the unique symmetric diffusion on $X$ which is locally isotropic. (For an analogous theorem for the Sierpinski gasket see [BP].) If we knew this, then any two limit points of $\mathbb{P}_{n}^{x}$ could differ only by a deterministic time change, and also, more significantly, we would know that any other construction of a symmetric locally isotropic diffusion on $\tilde{F}$ (such as the one given in [KZ] using random walks) would lead to essentially the same process.

We remark here that Osada [O2] has recently defined a diffusion $Z=\left((0)^{x}, Z_{t}\right)$ on $F$ which is not equal in law to $X$ and does not satisfy (5.18), but which does satisfy a more limited kind of invariance under local isometries. Let $S_{1}, S_{2} \in \mathcal{S}_{n}$, write $A_{i}=\operatorname{int}\left(S_{i}\right)$, and suppose that $\varphi$ is an isometry between $S_{1} \cap F$ and $S_{2} \cap F$. Then for $x \in A_{1} \cap F$ one has

$$
\mathbb{O}^{x}\left(\varphi\left(Z_{t \wedge \tau\left(A_{1}\right)}\right) \in \cdot, t \geq 0\right)=\mathbb{O}^{\varphi(x)}\left(Z_{t \wedge \tau\left(A_{2}\right)} \in \cdot, t \geq 0\right) .
$$

The key difference between (9.3) and (5.18) is that in (9.3) the processes are stopped on hitting the boundary of the cubes $S_{i}$.

This example shows that the definition of "locally isotropic" is quite sensitive.

See $[\mathrm{Sa}]$ and $[\mathrm{Me}]$ for some uniqueness results in the finitely ramified case.

3. Continuity of Harmonic Functions Let $x_{0} \in \tilde{F}$ and set $D=D_{n}\left(x_{0}\right) \cap \tilde{F}, D^{\prime}=D_{n+2}\left(x_{0}\right)$. Let $h$ be non-negative and harmonic in $D$. Then we know by Theorem 4.2 that there exists $\alpha>0$ such that $h$ is Hölder continuous of order $\alpha$ in $D^{\prime}$. What is the correct Hölder exponent for $h$ ? The constant $\alpha$ given in Theorem 4.2 comes from the Harnack inequality, so would not be a good estimate. If $d_{s}<2$ then the estimates of Section 4 of [BB3] imply that $h$ is Hölder continuous of order $d_{w}-d_{f}$. Even here, though, we do not know if that is best possible.

\section{References}

[AO] S. Alexander and R. Orbach, Density of states on fractals: "fractons". J. Physique (Paris) Lett. 43(1982), L625-L631.

[A] D. G. Aronson, Bounds on the fundamental solution of a parabolic equation. Bull. Amer. Math. Soc. 73(1967), 890-896.

[Bar1] M. T. Barlow, Diffusions on fractals. Lectures on Probability Theory and Statistics, École d'Été de Probabilités de Saint-Flour XXV - 1995, 1-121. Lecture Notes in Math. 1690, Springer 1998.

[BB1] M. T. Barlow and R. F. Bass, The construction of Brownian motion on the Sierpinski carpet. Ann. Inst. H. Poincaré Probab. Statist. 25(1989), 225-257.

[BB2] Local times for Brownian motion on the Sierpinski carpet. Probab. Theory Related Fields 85(1990), 91-104.

[BB3] On the resistance of the Sierpinski carpet. Proc. Roy. Soc. London Ser. A 431(1990), 345-360. 
[BB4] Transition densities for Brownian motion on the Sierpinski carpet. Probab. Theory Related Fields 91(1992), 307-330.

[BB5] Coupling and Harnack inequalities for Sierpinski carpets. Bull. Amer. Math. Soc. 29(1993), $208-212$.

[BB6] Random walks on graphical Sierpinski carpets. In: Proceedings of the Conference "Random walks and Discrete Potential Theory", Cortona 1997, to appear.

[BBS] M. T. Barlow, R. F. Bass, and J. D. Sherwood, Resistance and spectral dimension of Sierpinski carpets. J. Phys. A 23(1990), L253-L258.

[BP] M. T. Barlow and E. A. Perkins, Brownian motion on the Sierpinski gasket. Probab. Theory Related Fields 79(1988), 543-623.

[Bas1] R. F. Bass, Uniqueness for the Skorokhod equation with normal reflection in Lipschitz domains. Electron. J. Probab. (11) 1(1996), 1-29.

[Bas2] Probabilistic Techniques in Analysis. Springer, New York, 1995.

[Bas3] — Diffusions on the Sierpinski carpet. In: Trends in Probability and Related Analysis, World Scientific, Singapore, 1997, 1-34.

[BH] R. F. Bass and P. Hsu, Some potential theory for reflecting Brownian motion in Hölder and Lipschitz domains. Ann. Probab. 19(1991), 486-508.

[BK] R. F. Bass and D. Khoshnevisan, Local times on curves and uniform invariance principles. Probab. Theory Related Fields 92(1992), 465-492.

[BAH] D. Ben-Avraham and S. Havlin, Exact fractals with adjustable fractal and fracton dimensionalities. J. Phys. A. 16(1983), L559-563.

[BST] O. Ben-Basset, R. S. Strichartz and A. Teplyaev, What is not in the domain of the Laplacian on Sierpinski gasket type fractals? Preprint.

[Ca] L. Caffarelli, Métodos de continuação em equações eliticas não-lineares. Inst. Mat. Pura Apl., Rio de Janeiro, 1986.

[CKS] E. A. Carlen, S. Kusuoka, and D. W. Stroock, Upper bounds for symmetric Markov transition functions. Ann. Inst. H. Poincaré Probab. Statist. 23(1987), 245-287.

[CRRST] A. K. Chandra, P. Raghavan, W. L. Ruzzo, R. Smolensky and P. Tiwari, The electrical resistance of a graph captures its commute and cover times. Proceedings of the 21st ACM Symposium on theory of computing, 1989.

[Co] T. Coulhon, Dimension á l'infini d'un semi-groupe analytique. Bull. Sci. Math. 114(1990), 485-500.

[DS] P. Doyle and J. L. Snell, Random Walks and Electric Networks. Carus Math. Monographs, Math. Assoc. America, Washington DC, 1984.

[FS] E. B. Fabes and D. W. Stroock, A new proof of Moser's parabolic Harnack inequality via the old ideas of Nash. Arch. Rational Mech. Anal. 96(1986), 327-338.

[FHK] P. J. Fitzsimmons, B. M. Hambly and T. Kumagai, Transition density estimates for Brownian motion on affine nested fractals. Comm. Math. Phys. 165(1995), 595-620.

[FiS] P. J. Fitzsimmons and T. S. Salisbury, Capacity and energy for multiparameter Markov processes. Ann. Inst. H. Poincaré Probab. Statist. 25(1989), 325-350.

[Fuk] M. Fukushima, Dirichlet forms, diffusion processes, and spectral dimensions for nested fractals. In: Ideas and methods in stochastic analysis, stochastics and applications, Cambridge University Press, Cambridge, 1992, 151-161.

[FOT] M. Fukushima, Y. Oshima, and M. Takeda, Dirichlet Forms and Symmetric Markov Processes. de Gruyter, Berlin, 1994.

[GAM] Y. Gefen, A. Aharony, and B. Mandelbrot, Phase transitions on fractals: III. Infinitely ramified lattices. J. Phys. A 17(1984), 1277-1289.

[Go] S. Goldstein, Random walks and diffusion on fractals. In: Percolation theory and ergodic theory of infinite particle systems, Springer, New York, 1987, 121-129.

[Gr] A. A. Grigor'yan, The heat equation on noncompact Riemannian manifolds. Math. USSR Sbornik 72(1992), 47-77.

[H1] P. Hajłasz, Sobolev spaces on an arbitrary metric space. Potential Analysis 5(1996), 403-415.

[HHW1] K. Hattori, T. Hattori, and H. Watanabe, Gaussian field theories and the spectral dimensions. Progr. Theoret. Phys. Suppl. 92(1987), 108-143.

[HHW2] — New approximate renormalisation method on fractals. Phys Rev. A 32(1985), 3730-3733.

[HBA] S. Havlin and D. Ben-Avraham, Diffusion in disordered media. Adv. in Phys. 36(1987), 695-798.

$[\mathrm{Hu}] \quad$ J. E. Hutchinson, Fractals and self-similarity. Indiana J. Math. 30(1981), 713-747.

[Je] D. Jerison, The Poincaré inequality for vector fields satisfying Hörmander conditions. Duke Math. J. 53(1986), 503-523.

[Kig1] J. Kigami, A harmonic calculus on the Sierpinski space. Japan J. Appl. Math. 6(1989), 259-290.

[Kig2] A harmonic calculus for p.c.f. self-similar sets. Trans. Amer. Math. Soc. 335(1993), 721-755. 
[KM] J. Kinnunen and O. Martio, The Sobolev capacity on metric spaces. Ann. Acad. Sci. Fenn. Math. 21(1996), 367-382.

[Kum1] T. Kumagai, Estimates of the transition densities for Brownian motion on nested fractals. Probab. Theory Related Fields 96(1993), 205-224.

[Kum2] Regularity, closedness, and spectral dimension of the Dirichlet forms on p.c.f. self-similar sets. J. Math. Kyoto Univ. 33(1993), 765-786.

[Kus1] S. Kusuoka, A diffusion process on a fractal. Symposium on Probabilistic Methods in Mathematical Physics, Taniguchi, Katata. Academic Press, Amsterdam, 1987, 251-274.

[Kus2] Diffusion processes on nested fractals. Statistical Mechanics and Fractals. Springer, Berlin, 1993.

[KZ] S. Kusuoka and X. Y. Zhou, Dirichlet form on fractals: Poincaré constant and resistance. Probab. Theory Related Fields 93(1992), 169-196.

[L] T. Lindstrøm, Brownian motion on nested fractals. Mem. Amer. Math. Soc. 420(1990).

[Lv] T. Lindvall, Lectures on the coupling method. Wiley, New York, 1992.

[LR] T. Lindvall and L. C. G. Rogers, Coupling of multi-dimensional diffusions by reflection. Ann. Probab. 14(1986), 860-872.

[Man] B. Mandelbrot, The Fractal Geometry of Nature. W. H. Freeman, San Francisco, 1982.

[Ma] V. G. Maz'ja, Sobolev Spaces. Springer, New York, 1985.

[McG] I. McGillivray, Some applications of Dirichlet forms in probability theory. Ph.D. dissertation, Cambridge University, 1992.

[Me] V. Metz, How many diffusions exist on the Vicsek snowflake? Acta Appl. Math. 32(1993), $227-241$.

[M] J. Moser, On Harnack's inequality for elliptic differential equations. Comm. Pure Appl. Math. 14(1961), 577-591.

[Ny] S. O. Nyberg, Brownian motion on simple fractal spaces. Stochastics Stochastics Rep. 55(1995), 21-45.

[O1] H. Osada, Isoperimetric dimension and estimates of heat kernels of pre-Sierpinski carpets. Probab. Theory Related Fields 86(1990), 469-490.

[O2] Personal communication.

[RT] R. Rammal and G. Toulouse, Random walks on fractal structures and percolation clusters. J. Physique Lettres 44(1983), L13-L22.

[RS-N] F. Riesz and B. Sz.-Nagy, Functional Analysis. Ungar, New York, 1955.

[Ro] L. C. G. Rogers, Multiple points of Markov processes in a complete metric space. Séminaire de Probabilités XXIII, Springer, Berlin, 1989, 186-197.

[Sa] C. Sabot, Existence and uniqueness of diffusions on finitely ramified self-similar fractals. Ann. Sci. École Norm. Sup. 30(1997), 605-673.

[SC] L. Saloff-Coste, A note on Poincaré, Sobolev, and Harnack inequalities. Internat. Math. Res. Notices (1992), 27-38.

[Sie] W. Sierpinski, Sur une courbe cantorienne qui contient une image biunivoque et continue de toute courbe donnée. C. R. Acad. Sci. Paris 162(1916), 629-632.

[Tet] P. Tetali, Random walks and the effective resistance of networks. J. Theoret. Probab. 4(1991), 101-109.

[V1] N. Th. Varopoulos, Isoperimetric inequalities and Markov chains. J. Funct. Anal. 63(1985), $215-239$.

[V2] Random walks and Brownian motion on manifolds. Sympos. Math. 29(1987), 97-109.

[Z] X. Y. Zhou, On the recurrence of simple random walk on fractals. J. Appl. Probab. 29(1992), 460-466.

Department of Mathematics

University of British Columbia

Vancouver, BC

V6T $1 Z 2$
Department of Mathematics

University of Connecticut

Storrs, CT 06269

USA 